\title{
Polymers Division Fy 2003 Programs and Accomplishments
}
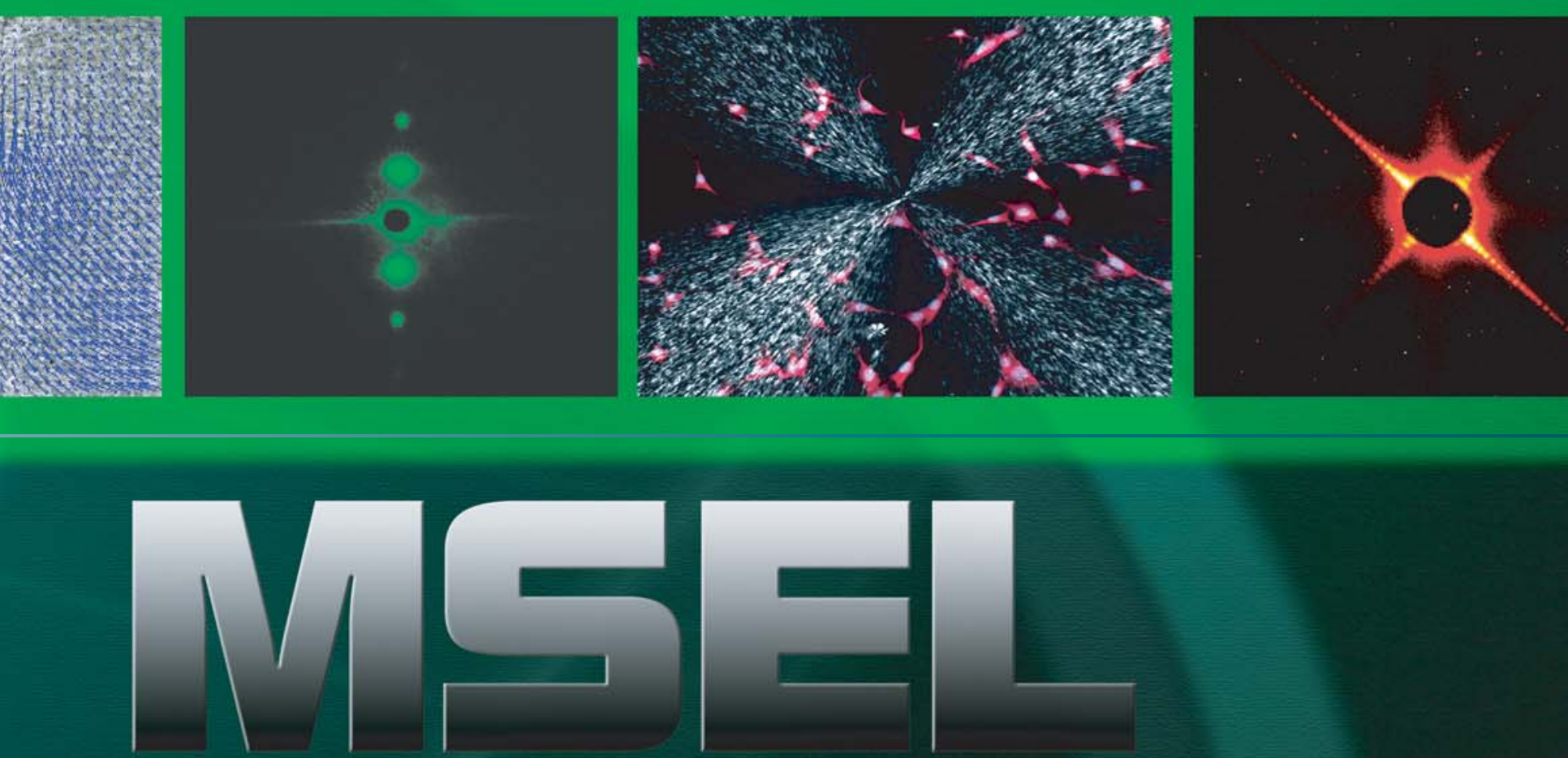

\section{NG}

National Institute of

Standards and Technology

Technology Administration

U.S. Department of

Commerce

NISTIR 7016

September 2003 


\section{On the Cover:}

From the front cover and continuing on to the back, the images shown are, respectively:

Fig 1 - A 2-dimensional small angle x-ray scattering (SAXS) detector image obtained from the transmission measurement of a dense array of via-pads (approximated as a brick pattern) etched into silicon oxide;

Fig 2 - Osteoblasts growing on a poly(L-lactide) (PLLA) spherulite;

Fig 3 - Light diffraction patterns from a strain induced buckling pattern in polystyrene; and

Fig 4 - A micrograph of a microchannel analog of the 4-roll mill showing the measured velocity vectors of particles in the device under flow.

More information about each of these topics can be found within this report. 
National Institute of

Standards and Technology

Arden L. Bement, Jr.

Director

Technology

Administration

Phillip J. Bond

Undersecretary of

Commerce for Technology

U.S. Department

of Commerce

Donald L. Evans

Secretary

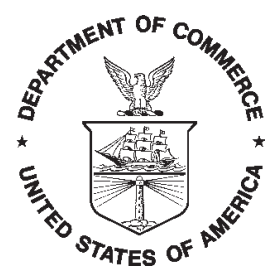

\section{Materials Science and Engineering Laboratory}

\section{FY 2003 Programs and AccomplishmeNTS}

\section{Polymers Division}

Eric J. Amis, Chief

Chad R. Snyder, Deputy 



\section{Table of Contents}

Executive Summary 1

Technical Highlights:

Reaching Outwards 3

A Fully Automated Peak Picking and Integration Algorithm for Mass Spectral Data

Pore Size Distributions in Low-k Dielectric Thin Films

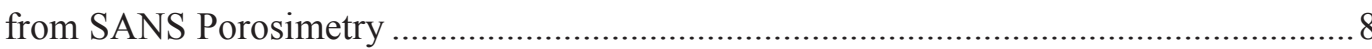

Critical Dimension Metrology of Nanoscale Structures with

Small Angle X-ray Scattering 10

High-throughput Measurements of Elastic Moduli of Polymer Thin Films 12

Elastic Flow Instability in Polymer-Dispersed Carbon Nanotubes 14

Scaffold Structure and Cell Function through Multi-Modal Imaging and Quantitative Visualization .16

Macromolecular Dynamics via Inelastic Neutron Scattering 18

Advanced Manufacturing Methods .21

Processing Flows \& Instabilities .22

Combinatorial Methods .23

Polymer Formulations: Fluidic Devices and Scattering

Measurements for High Throughput Materials Research

Combinatorial Epoxy Curing Library: Correlating Network

Formation, Chemical Conversion, and Adhesion .25

NIST Combinatorial Methods Center (NCMC)

A Partner in Accelerating Materials Research .26

Interface of Materials with Biology

Bioactive Polymeric Composites for Mineralized

Tissue Regeneration 28

High-Throughput Biocompatibility Screening of Polymer Blends

Measurement of Cellular Response to Three-Dimensional

Scaffolds for Tissue Engineering 


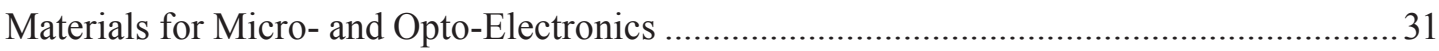

Characterization of Porous Low-k Dielectric Constant Thin Films .................................. 32

Polymer Photoresists for Next-Generation Nanolithography ...........................................33

Dielectric Measurements of Polymer Composite Films

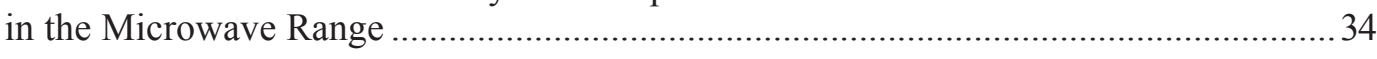

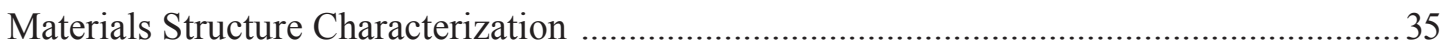

Coherent Anti-Stokes Raman Micro-spectroscopy ( $\mu$-CARS)

for Understanding Tissue Growth in Scaffold Constructs ................................................... 36

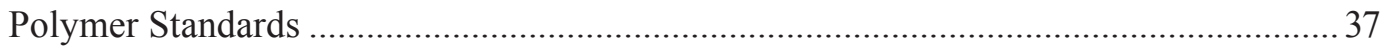

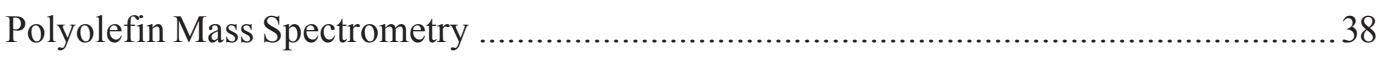

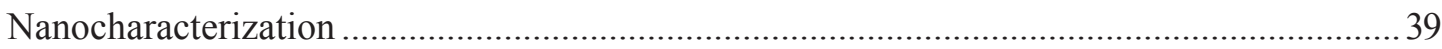

Process Monitoring of Polymer Clay Nanocomposites ................................................... 40

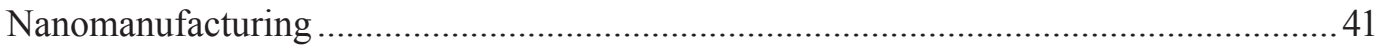

Polymers Division FY03 Annual Report Publication List ......................................................... 43

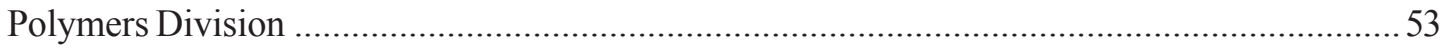

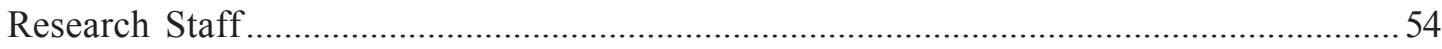

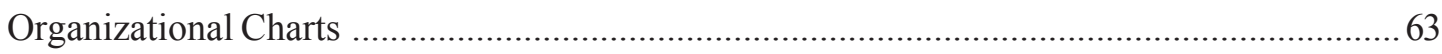




\section{Executive Summary}

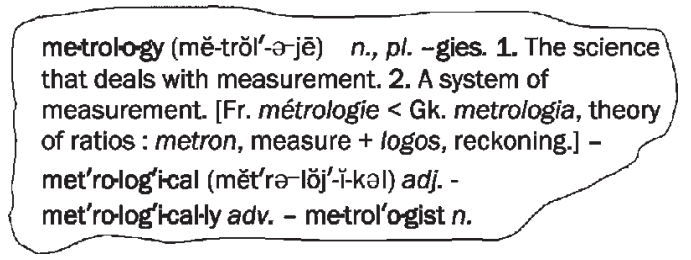

Measurement is at the core of understanding, and at NIST, we take pride in both our fundamental metrology and our creative investigations to establish the essential underlying scientific foundation. In both respects, I am pleased to report that this has been an excellent year for the scientists in the NIST Polymers Division. As usual, this annual report is organized to briefly describe the major project areas where we have focused our efforts within the programs of the Materials Science and Engineering Laboratory. In addition, we have highlighted several specific successes within the NIST 2010 Strategic Focus Areas of information and knowledge management, nanotechnology, and health care.

Information and knowledge management are critical to a metrology system both for cataloging and for interpreting data. Our first technical highlight addresses this issue for mass spectrometry by introducing a robust data analysis tool with no user-selected parameters that has been developed in collaboration with the NIST Information Technology Laboratory. The benefits to this algorithm are that it eliminates user bias from the analysis, and it allows automated processing of large quantities of data. In addition to advancing our goal of developing absolute molecular mass Standard Reference Materials, and extending the reach of polymer mass spectrometry for advanced quantitative applications, we have already seen that this tool will be used in the growing application of mass spectrometry for proteomics research.

The National Nanotechnology Initiative identifies Nanoscale Instrumentation and Metrology as one of the "Grand Challenges," and in this area, NIST has a major role. This year, four of our highlights focus on this grand challenge. To aid the semiconductor industry in their search for new low-k dielectric materials, we have extended our definitive characterization work with new methods to determine the distribution of pore sizes and to reveal extremely subtle material characteristics that have been inaccessible by other measurements. For the lithographic process itself, we have developed new techniques to characterize pattern uniformity and defects even for the dense and high aspect ratio patterns that will enable the next generation of semiconductors and nanotechnology. It is also clear that as electronic devices continue to shrink, their mechanical properties will be increasingly important. There have been a number of approaches to this problem, and our contribution this year raises the bar with a unique method that provides rapid and quantitative characterization of the elastic modulus of supported films. Finally, we have taken advantage of our expertise in multiphase flow to describe the critical effects of processing on the dispersion and orientation of multiwall carbon nanotubes in polymer solutions.

In the third NIST Strategic Focus Area of health care, we are highlighting two relatively new projects related to tissue engineering, which is a major emphasis for NIST that was announced in our report last year. The first of these projects originated from the non-destructive imaging method of optical coherence tomography that we had used for studies of polymer-fiber composites. We have now extended the method, based on a combination of optical coherence and confocal fluorescence microscopy, to develop powerful tools for in situ measurements of 3D structure and biological activity within tissue engineering scaffolds. In addition to the qualitative trends that can be studied by simply observing the images, we are demonstrating techniques capable of extracting quantitative, chemically specific data from the images. The final highlight describes a study, completed at the world-class inelastic neutron scattering facilities of the NIST Center for Neutron Research, to observe dynamics in macromolecular materials. From these measurements, we are able to investigate the mechanisms of cryopreservation used for stabilization of proteins, pharmaceuticals, and biomaterials. Although these are early stage projects, both of them demonstrate how we expect to leverage state-of-the-art measurement science at NIST to address critical needs for biomedical materials.

The Polymers Division has now grown to over 100 staff, postdoctoral associates, and guest researchers, and together, we continue to foster productive and dynamic project teams. We take pride in the creativity of NIST scientists, from the most senior staff of 44 years to the newest postdoctoral researcher who arrived last week. It is always difficult for me to choose from all that was completed in the past year for the few items we can highlight. Please visit our website for more details on current work, copies of our publications, and to check on a new link that we refer to as "Out-from-Under-the-Radar." As always, I welcome your comments.

Eric J. Amis

Chief, Polymers Division 



\section{Reaching Outwards. . .}

\author{
"If a tree falls in the forest and no one is there \\ to hear it, does it really make a sound?" \\ "If research is performed and no one is there
to hear it, does it really matter?"
}

A strong outreach effort assures that people are hearing about what you are doing. It also should determine if they actually CARE about what you are doing.

$\mathrm{W}_{\mathrm{c}}$ ho cares?" is a question that arises when considering the impact of science and engineering.

To ensure that people do care about, and are aware of, the activities of our Division, we maintain a rather strong outreach program. In considering outreach efforts, the three obvious "modes" include disseminating, collaborating, and exchanging. Dissemination is one of the most popular techniques for outreach in the physical sciences. The paramount example of the dissemination mode is publication in peer-reviewed archival journals. The limitation to this method, of course, is that you can never really be sure how many people read and learned from the papers. Like other researchers, we do exploit this method of outreach, and our publication rate is high. However, there are several other avenues that we exploit including NIST Special Publications and web services of various types. The collaborative mode of outreach is probably the most productive method of outreach. This method includes workshops, standards committees, special interest groups, and partnerships. The last mode of outreach is exchange. This mode tends to generate long-term partnerships and collaborations; examples of this range from lecturers who we have invited to give presentations to us to summer students who gain laboratory experience at an early age. On the following pages, we will present specific examples of our outreach efforts to give you a better understanding of exactly how we do business.

\section{Dissemination}

This year, we published a NIST Recommended Practice Guide, Capacitance Cell Measurement of the Out-of-Plane Expansion of Thin Films (NIST SP 960-7). This guide is intended for use in microelectronics where accurate measurement of thermal expansion is important in the design of interlevel dielectrics. This guide was developed to make this sensitive metrology more accessible by detailing the construction and use of the capacitance-based metrology for thermal expansion measurements. It has been made available as both a hard copy guide and electronically on the web. We are also using the web to further disseminate our research by posting our publications on our website (www.nist.gov/polymers). The database is searchable by research group, year of publication, subject term, author, and keyword. To complement the publications, we also post information regarding our ongoing research projects, highlights of recent research, and information on our staff's research interests and expertise. Additionally, we have added a database that consists of methods, taken largely from the peer-reviewed scientific literature, for matrix-assisted laser desorption ionization (MALDI) mass spectrometry on a wide variety of synthetic polymers. The current listing contains over 100 recipes. We encourage you to visit our website to learn more about these items.
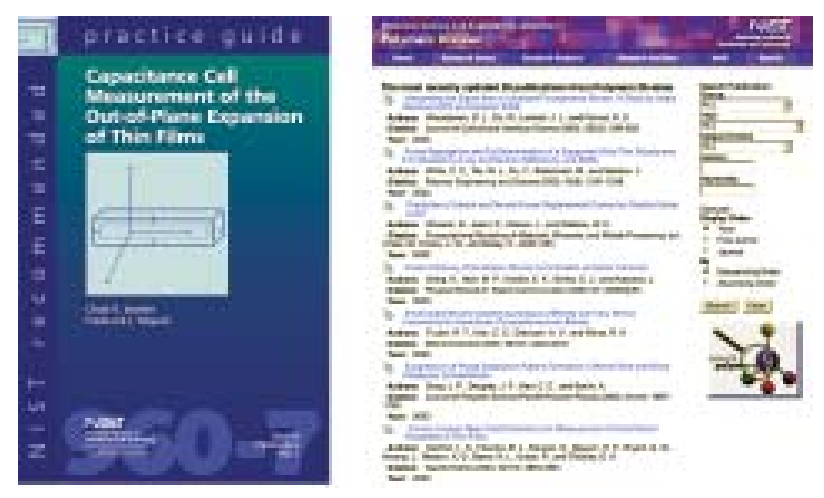

\section{Collaboration}

One of the most prominent examples of the collaborative mode during the past fiscal year was the large number of workshops ranging across most of the Division's programmatic areas. To demonstrate the continuous nature of this effort, we have listed the workshops chronologically, rather than programmatically.

\section{Workshops}

On October 7-8, 2002, the NIST Combinatorial Methods Center (NCMC) held its second member's workshop entitled "Adhesion and Mechanical Properties." Over 40 member representatives attended the workshop from 14 major industrial companies. The first day focused on recent advances in combinatorial adhesion testing developed by the NCMC, including combinatorial peel testing and a multilens contact adhesion test. The second day centered on the topic of mechanical properties. The morning session concluded with practical laboratory demonstrations of the NCMC's gradient library fabrication techniques, analysis and instrumentation including flow coating, 
composition gradient libraries for viscous formulations, combinatorial lens adhesion measurements, combinatorial peel testing, and polymer composition gradients using melt extrusion. The afternoon session consisted of a panel discussion focusing on member needs and future directions of the NCMC.

On November 7-8, 2002, experts in the field of mass spectrometry met at NIST to discuss measurement barriers to quantification of molecular characteristics of synthetic polymers. It also featured a problem-solving session in which industrial users of the method presented measurement challenges and problems specific to their applications.

The workshop was organized according to key steps in the MALDI-MS measurement process - sample preparation, volatilization and ionization, ion detection and data analysis. Participants concluded that experimental confirmation of key molecular events during the volatilization and ionization processes was needed. A report on the workshop is published in the NIST Journal of Research, available on the Web.

On January 15, 2003, Polymers Division researchers were invited to International SEMATECH (ISMT), an industrial consortium of semiconductor manufacturing companies, to present results from unique measurement methods for the evaluation of materials factors impacting the ultimate resolution of next-generation photoresists to its Resist Advisory Group. The Resist Advisory Group audience represents a large fraction of the companies directly involved with the development and production of the sub-100 $\mathrm{nm}$ structures in current, integrated circuits. Interaction with the participants provided useful feedback on the timeliness, relevance, and quality of the measurement methods developed by NIST to the further development of next-generation lithographic materials.

On January 27-28, 2003, a two-day workshop on scanning probe microscopy (SPM) was held at NIST. SPM techniques are especially good for examining materials locally for a wide range of material properties, including surface topology, surface tribology, elastic properties, ferroelectric domain structure, electric potential and impedance at surfaces, and the atomic structure of crystals at surfaces. The primary purpose of the workshop was to familiarize people with the many uses of the technique. Guests from government labs included the FDA, USDA, and NRL, and guests from industry included GE and Millenium Chemicals.

On February 19, 2003, the NCMC co-organized a full day workshop that was held in conjunction with the Knowledge Foundation's "COMBI 2003 Combinatorial Approaches for New Materials Discovery" meeting (February 20-21, 2003, in San Jose, CA). The NCMC pre-conference seminar entitled, "High-Throughput Materials Informatics:
Knowledge Acquisition for Better Materials," was attended by over 30 representatives from industry and academia. The seminar examined issues concerning the enormous amounts of data created by combinatorial/high-throughput materials research and the conversion of such data into usable information.

On March 20-21, 2003, the Polymers Division hosted the second "NIST-KIPS Symposium on Polymer Science." (KIPS is the acronym for the Kyoto Institute of Polymer Science.) This bi-annual conference/workshop builds bridges and fosters collaborations between polymer scientists in the U.S. and those in Japan. Approximately 100 attendees, including 16 from KIPS and the remainder from U.S. universities and NIST, participated in intensive lectures, discussions, and poster sessions which covered the full range of polymer science.

On May 22-23, 2003, the NCMC held its third member's workshop entitled "Combinatorial Informatics." Over 30 member representatives from 16 companies and universities and 26 NIST staff attended the workshop. The goal of the workshop was to illuminate needs in combinatorial and high-throughput informatics research, in particular in combi-oriented data standards called for by the NCMC industrial community. The workshop culminated in a panel discussion aimed at initiating a process for the establishment of mutually beneficial data interchange standards. As a result, the Combinatorial Materials Research Data Standards (CMRDS) Working Group was formed. The final sessions of the workshop concerned NCMC research activities and services. Here, the laboratory informatics system being developed at the NCMC core facility was described.

On June 5, 2003, two Polymers Division researchers presented invited lectures on recent advances in the characterization of the structure and properties of nanoporous low-k dielectric materials at the "Low-k Porosimetry Workshop" sponsored by ISMT in June 2003. The NIST presentations complemented other presentations from university, industry, and governmental laboratories. NIST participation in the workshop helped facilitate discussion of all available measurement methods for characterizing next-generation dielectric materials.

\section{Standards Developing Organizations (SDO's)}

A major part of NIST's Congressionally mandated mission is the development of standards to improve commerce and facilitate trade. For this reason, one of our more active collaborative outreach methods is through our participation in standards committees. Rather than enumerate all of our memberships, we have chosen a few examples that range across our programmatic areas with different levels of participation. 
A draft standard protocol for determination of molecular mass and molecular mass distribution of atactic polystyrene by matrix-assisted laser desorption/ionization - time of flight - mass spectrometry has been distributed to members of ISO/TC 35/SC 10 on June 6, 2002 for balloting and is also under review by ASTM. The standard method will be useful in comparisons of mass spectrometers, sample preparation for mass spectrometry, and data analysis. The method was developed under the auspices of the Versailles Project on Advanced Materials and Standards (VAMAS) by an international team led by a researcher from the Polymers Division. The protocol is derived from a procedure used in an international interlaboratory comparison of analysis of polystyrene by MALDI MS. The polystyrene analyzed in the interlaboratory comparison was also issued as a NIST Standard Reference Material in FY 2003.

A Polymers Division researcher chairs an IPC task group for the development of standard test methods for embedded passive devices. In this role, NIST guides industrial partners in developing and drafting standard test documents and manuals for this emerging materials area. This close working arrangement allows for direct feedback of standard measurement method development that will positively impact the electronics industry. In addition, new technical challenges have furthered the development of needed standard test methods.

A wide variety of standards-related activities are ongoing in the area of biomedical devices and materials. Polymers Division researchers are actively involved in ASTM committee F04 (Medical and Surgical Materials and Devices) to expedite introduction of reference tissue scaffolds to aid in the development of characterization methods for their structure and properties. Additionally, Division researchers have been involved in planning an ASTM workshop on cleanliness of medical implants in 2003, a symposium on cell-materials signaling in 2003, and several other workshops and symposia in future years. Further information on health care related standards in the Division is provided in the Polymer Standards project write-up.

\section{Special Interest Groups (SIGs)}

One of the collaborative methods that we use rarely, and only when it is appropriate, is through SIGs. One example of this can be found in our Processing Characterization efforts. Due to the complexity of the phenomena occurring during polymer manufacturing, precise measurements of polymer processing parameters is difficult. Yet the ability to precisely measure these properties is necessary in order to achieve six sigma manufacturing goals. In recognition of this problem, a Polymers Division scientist has established a SIG devoted to real-time measurement issues. This group meets every year at the Society of Plastics Engineer's annual technical meeting. At this year's meeting, 15 papers were presented on process monitoring using a variety of techniques, such as ultrasonics, fluorescence, and analytical mass spectroscopy. Dissemination of these on-line measurement concepts through the SIG allows manufacturers to achieve greater quality control.

\section{Exchange}

In FY 2003, we had 35 invited lecturers in to give presentations on cutting edge research in all of the Division's programmatic areas as well as in several new strategic directions. As a complement to the information provided in the lectures, informal meetings are typically held during the remainder of the day for our staff to exchange information with the visitor. In addition to these short-term exchanges, every year we bring in students to collaborate on research projects. The students have varying levels of experience, ranging from high school students through undergraduates to graduate students. By way of example, we hosted three students from Montgomery Blair High School (Montgomery County, MD) this year. Two assisted in polymers formulations research, while another returned to co-author an invited article for Microscopy Today based on his work from the previous summer. In addition, a University of Maryland Baltimore County (UMBC) undergraduate, introduced to NIST through the Minority Access Program, worked on combinatorial informatics and mechanical properties projects. We also have active collaborations with guest researchers from other institutions, and through the National Research Council (NRC)/NIST postdoctoral research program, we are constantly exchanging new ideas and techniques. In the past two fiscal years, we have received 18 new postdocs through this program. Finally, this year NIST and the National Institutes of Health (NIH) have initiated a joint postdoctoral research program, and several Division researchers have research opportunities through this program. (Further information is available about the joint postdoctoral program on the National Academies website at www.nationalacademies.org by searching for "NIH/NIST.")

\section{A Final Note. . .}

As you may have noticed, we left out one noticeable example of outreach, and that is through the publication of our Annual Report. We hope that you enjoy the following technical highlights and project reports, and we look forward to reaching out to you further in the future.

\section{For More Information on this Topic}

Chad R. Snyder (Polymers Division, NIST)

www.nist.gov/polymers 


\section{A Fully Automated Peak Picking and Integration Algorithm for Mass Spectral Data}

\begin{abstract}
A numerical algorithm is described that accurately locates and calculates the area beneath peaks from real mass spectral data using only reproducible mathematical operations and no user-selected parameters. Such a fully automated algorithm was required for rapid and repeatable processing of mass spectral data containing hundreds of peaks. By working without any user input, it both saves operator time and eliminates operator bias. The first criterion is desirable when processing large amounts of data (for example in proteomics research). The second criterion is necessary to the Polymer Division's goal of creating an absolute molecular mass distribution synthetic polymer Standard Reference Material where operator bias in the data analysis cannot be tolerated.
\end{abstract}

\section{William E. Wallace and Anthony J. Kearsley}

\begin{abstract}
A unified collection of algorithms has been developed that accurately locates peaks and calculates their area using only reproducible mathematical operations and no user-selected parameters. As shown in Figure 1, the method consists of three steps: 1) statistical characterization of the data set and an analyte-free background spectrum; 2) data set segmentation to determine "strategic points"; and 3) deflation of the number of strategic points guided by the statistical properties of the data sets. The final deflated set of strategic points consists of groups of three points that define the beginning, center, and end of each peak in the data. For closely spaced peaks, the strategic point that defines the end of one peak may also define the beginning of the next. Finally, a polygonal fitting routine is used to calculate relative peak area.
\end{abstract}

The time-series segmentation algorithm at the heart of the method consists of two steps. The first portion (2a) requires the selection of the strategic points. These points are selected based on an iterative procedure that identifies points whose orthogonal distance from the end-point connecting line segment is greatest. Once a point with greatest orthogonal distance from the mean has been identified, it joins the collection of strategic points and, in turn, becomes an end-point for two new line segments from which a point with greatest orthogonal distance is again found.
This numerical scheme is performed until the greatest orthogonal distance to any end-point connecting line segment drops beneath a prescribed threshold value. This threshold value is calculated from the statistical properties of the data set. The selection of these points does not require equally spaced data. The second phase of the algorithm (2b) requires the solution of an optimization problem, specifically, locating strategic point heights (that is, adjusting strategic y-axis values associated with strategic $\mathrm{x}$-axis values) that minimize the sum of orthogonal distance from raw data. This problem is a nonlinear (and non-quadratic) optimization problem that can be accomplished quickly using a modern nonlinear programming algorithm. Parts $2 \mathrm{a}$ and $2 \mathrm{~b}$ are collectively called the Kearsley-Wallace method, which is an extension of the earlier Douglas-Peucker method.

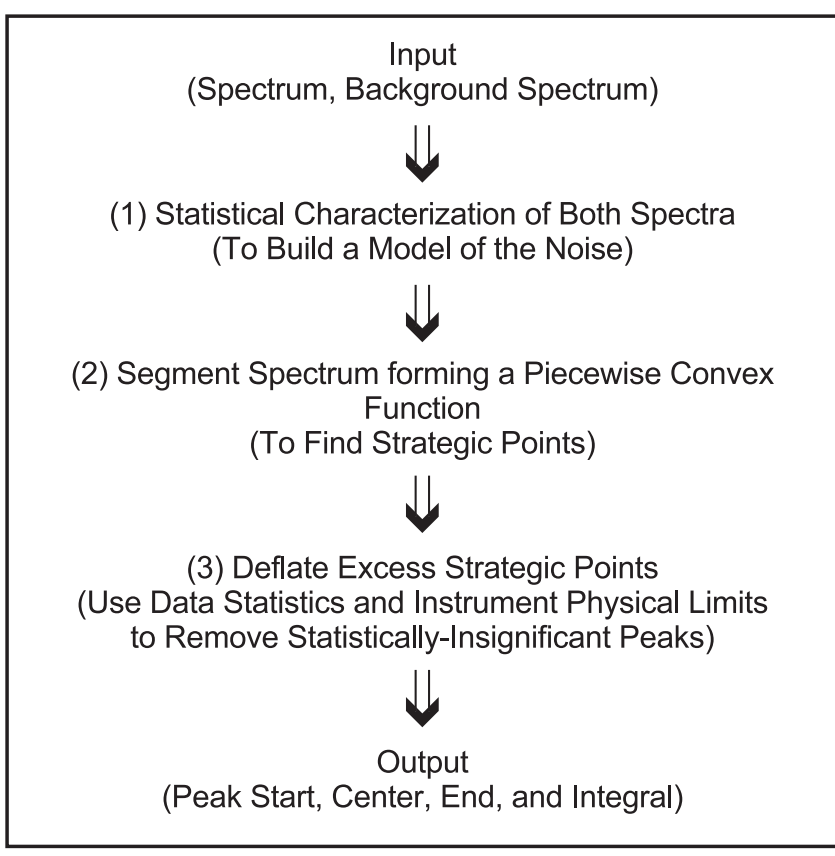

Figure 1: Method Flow Chart.

Consider the polystyrene matrix-assisted laser desorption ionization time-of-flight mass spectrum shown in Figure 2 (black) and its complementary matrix-only background spectrum (red). The resultant strategic points (green) defining peak beginning, center, and end, and the relative peak areas (blue) are also shown. Note that ion intensity is on a logarithmic scale, 
thus the small peaks are significantly smaller than the main series of peaks. The analysis of this was done without operator intervention of any sort. The only input provided was the spectrum to be analyzed and an analyte-free spectrum to determine inherent instrument noise. The noise has both chemical (e.g., improperly time-focused ions) and electronic (e.g., detector dark current) components. These noise elements span a wide frequency range and cannot simply be smoothed out of the data without distorting peak shape (and, therefore, peak area). Our experience shows that the power

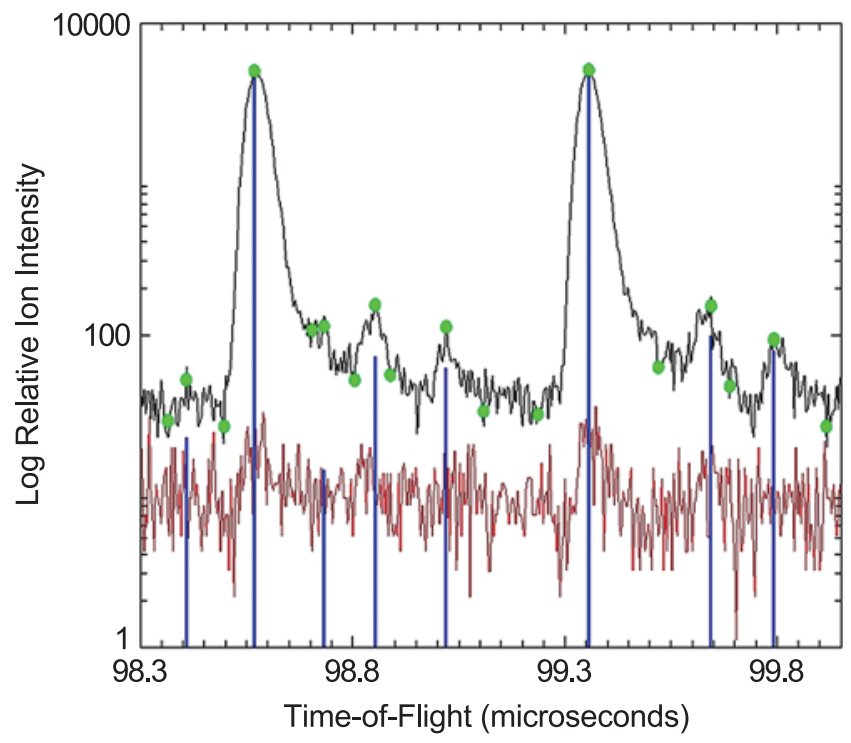

Figure 2: Sample polystyrene MALDI TOF mass spectrum (black) and its complementary matrix-only background spectrum (red). spectrum of the noise cannot be predicted solely from the experimental conditions; therefore, blind application of smoothing and/or filtering algorithms will unintentionally remove information from the data.

Some of the additional strengths of this method include the fact that it requires no knowledge of peak shape, and, furthermore, it requires no preprocessing of the data, i.e., smoothing or baseline correction with their resultant distortion of peak area. Lastly, the method does not require equal spacing of data points (e.g., time-of-flight data can be processed in mass-space where the points have a square root spacing). The one significant weakness is that the method is more successful and efficient if a blank (analyte-free) spectrum is used to calibrate instrument background noise. (However, such a background spectrum is not strictly required.)

Future plans include the creation of a publicly accessible, secure Web-server application for on-line, real-time application of the algorithm. We will also relay the method to other standards-setting organizations for comment and to commercial software vendors for implementation in their products. Lastly, we have begun to tackle the much more subtle problem of automated, operator-independent baseline compensation.

\section{For More Information on this Topic}

W.E. Wallace, C.M. Guttman (Polymers Division, NIST); A.J. Kearsley, J. Bernal (Mathematical and Computational Sciences Division, NIST) 


\section{Pore Size Distributions in Low-k Dielectric Thin Films from SANS Porosimetry}

The microelectronics industry is testing a wide variety of porous low-dielectric constant ("low-k") materials for future use in integrated circuits. To understand low-k thin film properties, a quantitative analysis of pore size distribution is vital. The Electronics Materials group has developed a new approach to this challenging problem based on a small angle neutron scattering (SANS) porosimetry technique. The new technique quantifies pore size distribution and reveals subtle material characteristics inaccessible to other measurement techniques.

\section{Barry J. Bauer and Ronald C. Hedden}

$\mathrm{A}^{\mathrm{s}}$ feature size on integrated circuits decreases to below $100 \mathrm{~nm}$, electrical crosstalk, or interference between interconnects, becomes a major obstacle to device performance. A need has arisen to develop improved electrical insulating materials for the next generation of microelectronic devices. Silicon dioxide and fluorosilicate glass, the insulators used in today's devices, have dielectric constants $(\mathrm{k})$ of about 4.2 and 3.7 respectively, but materials with dielectric constants of 2.2 or lower will be needed by 2007 . However, it has proven difficult to improve existing materials by changing their chemical structure.

Much of the recent developmental efforts in low-k dielectrics involve production of nanoporous thin films. By introducing tiny air-filled pores into the material, the bulk dielectric constant is lowered because air has a dielectric constant of 1.0. The microelectronics industry is testing a wide variety of porous low-dielectric constant ("low-k") materials, including both organosilicate and organic polymer types. To understand the basis for their physical properties, detailed structural characterization is essential. Specifically, quantitative characterization of the pore size distribution is a critical issue because pore size impacts mechanical, thermal, and barrier properties of the films. Because pore size is very small $(<10 \mathrm{~nm})$ and pore volume fraction is high $(0.1$ to 0.5$)$, microscopy techniques are generally insufficient for evaluation of pore size distribution.

A new approach to pore size determination combines the established science of capillary porosimetry with a powerful analytical technique, small angle neutron scattering (SANS). Capillary porosimetry is an established experimental technique applicable to determination of pore size distributions. The porous material is exposed to the vapor of a "probe solvent," and pores fill with liquid solvent by capillary condensation. An adsorption isotherm is constructed by measuring solvent uptake as a function of partial pressure. In general, filling of the pores with solvent is size-dependent; the smallest pores fill first as solvent partial pressure is increased. Alternatively, the sample temperature may be varied while solvent partial pressure is held constant. Optical, x-ray, or gravimetric techniques may be employed to measure solvent uptake. Figure 1 shows solvent adsorption curves obtained by specular x-ray reflectivity (SXR) for an organosilicate type low-k material.

A limitation of capillary porosimetry is that pore size is usually not directly measured but is calculated by applying a physical model to the adsorption data. Serious ambiguities result when hysteresis phenomena are observed, which is normally the case for nanoporous materials. In addition, physical models for adsorption may fail when pores are very small, as in the low-k dielectrics. Conventional analysis of capillary porosimetry data, therefore, can provide only qualitative comparisons between low-k materials, not quantitative pore size distributions. However, a great improvement to the technique is possible by using small angle neutron

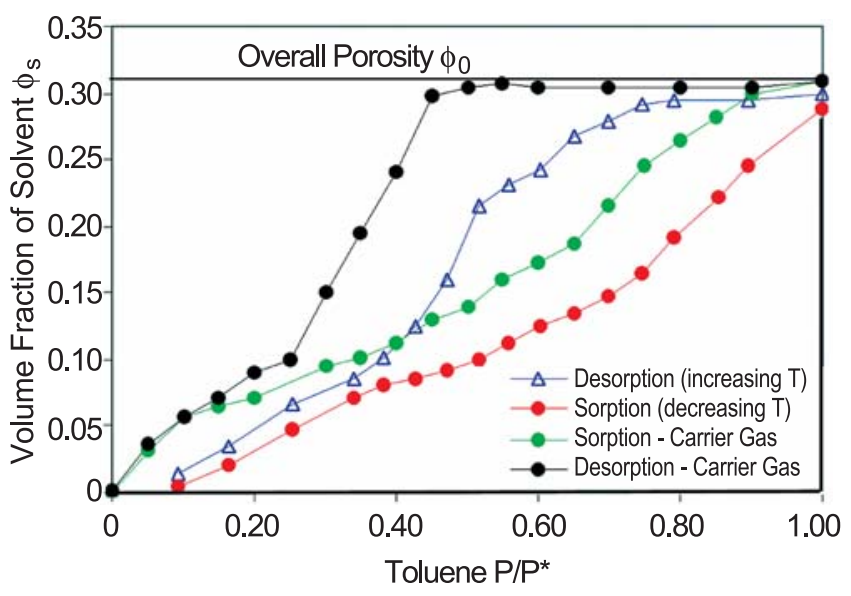

Figure 1: Solvent adsorption data for an organosilicate low- $k$ dielectric measured by $x$-ray reflectivity. Porosimetry is conducted by two methods: under isothermal conditions while varying solvent partial pressure in a carrier gas (green, black), or in a saturated solvent atmosphere while varying sample temperature (red, blue). 
scattering (SANS) to observe the adsorption process. By monitoring the pore filling and unfilling with SANS, important characteristics of the pore size distribution can be quantified irrespective of hysteresis phenomena.

To facilitate SANS porosimetry experiments, a programmable atmospheric control device was constructed that uses mass flow controllers to deliver solvent vapor in air to a flow-through sample cell. The partial pressure $P$ of the solvent can be varied from 0.0 (pure air) to $P^{*}$ (saturated solvent vapor). The sample may also be exposed to solvent vapor at a constant partial pressure $\mathrm{P}$ while sample temperature is varied, effectively changing $\mathrm{P}^{*}$.

The first stage of neutron porosimetry is a "contrast match point" determination. The pores are filled with solvent mixtures of variable neutron scattering length density (SLD). The solvent SLD is varied by mixing hydrogen- and deuterium-containing analogs of the solvent. The scattered intensity $I(q)$ from the solvent-filled, porous material depends on the difference in SLD or "contrast" between the solvent and the matrix. The solvent SLD is varied systematically, and the scattered intensity $I(q)$ is measured at each composition. If the material is homogeneous in atomic composition and all of the pores fill with solvent, a contrast match solvent composition exists for which $I(q)$ becomes zero over all $q$. If the material has inhomogeneities in its atomic composition, or if some of the pores are not filled by solvent ("closed pores"), a contrast match point will not be observed. SANS porosimetry is more applicable to samples with a true contrast match point, as subsequent data analysis is complicated by inhomogeneities.

The second stage of the measurements is porosimetry using solvent vapor of the contrast match composition. SANS data are collected as the partial pressure of the probe solvent is increased (sorption) and decreased (desorption). When pores fill with the contrast match liquid, their SLD matches the sample matrix, so the SANS measurement yields the size of only the "empty" pores. Thus, as solvent partial pressure is increased, pore size is measured for sub-populations of pores of increasingly large size.

If the volume fraction of the solvent in the film is also measured as a function of partial pressure, then average pore size and pore volume fraction are simultaneously known. X-ray reflectivity porosimetry allows such measurement with high precision and is a powerful complement to SANS porosimetry. Figure 2 shows combined results from both techniques: pore size from SANS is plotted vs. empty pore volume fraction from SXR. The data are essentially unaffected by hysteresis phenomena within the limits of uncertainty of the experiment. When all pores are empty, the SANS measurement gives the average pore size for the entire distribution. As pores are filled, the upper end of the pore size distribution is probed because only the larger pores are empty and contribute to $I(q)$ from SANS. For the sample shown in Figure 2, the average pore size is about $60 \AA$, but the sample contains some pores of $80 \AA$ or larger. By using capillary porosimetry to isolate the scattering from the largest pores, sub-populations of large pores within a low-k material can be readily identified, provided their concentration is high enough to make a measurable change in the scattering. Such information is valuable to the low-k integration effort because large pores are a leading cause of device failure.

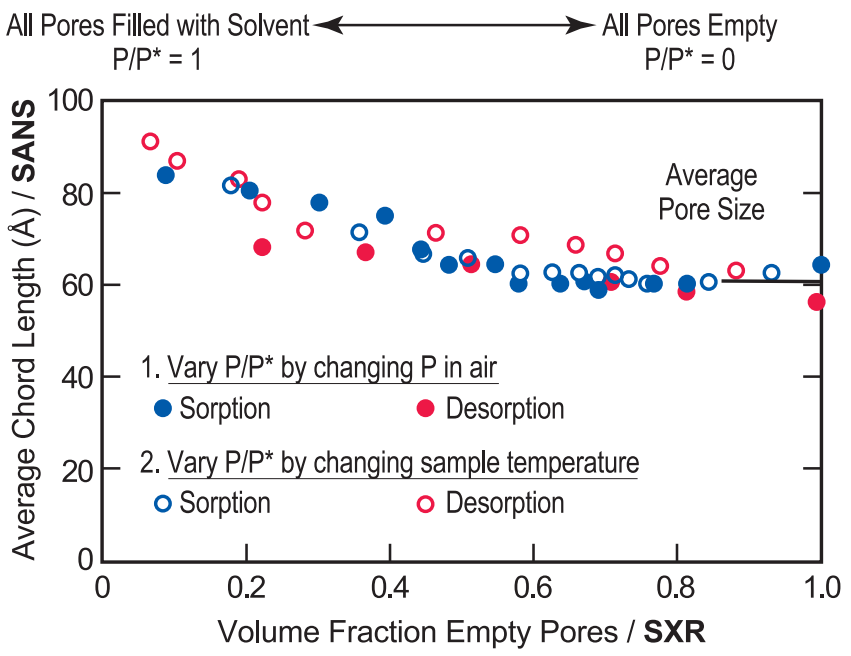

Figure 2: Size information (average chord length) from SANS porosimetry plotted $v$ s. volume fraction empty pores from $x$-ray reflectivity. The scattering data contain size information related to the average size of only the empty portion of the pores. As solvent partial pressure is increased from $P / P^{*}=0.0$ to $P / P^{*}=1.0$ (saturated solvent vapor), pores fill with liquid solvent in order from smallest to largest. As $P / P^{*}$ approaches 1.0, the scattering from the largest pores is probed.

The SANS porosimetry technique allows characterization of pore size distribution without a need for a physical model for adsorption. SANS porosimetry is perhaps the most quantitative technique available today for characterizing pore size distribution in nanoporous low-k thin films.

\section{For More Information on this Topic}

B. Bauer, H. Lee (Polymers Division, NIST); R. Hedden (Department of Materials Science and Engineering, The Pennsylvania State University) 


\section{Critical Dimension Metrology of Nanoscale Structures with Small Angle X-ray Scattering}

The continued reduction in pattern sizes throughout the semiconductor industry will soon require new metrologies capable of high throughput non-destructive measurements of dense, high-aspect ratio patterns with sub-nanometer resolution. In collaboration with industrial partners, we are developing a metrology based on Small Angle X-ray Scattering (SAXS) to quickly, quantitatively, and non-destructively measure the smallest, or "critical," dimensions expected in the next two technology nodes with sub-nanometer precision. Quantities of interest include critical dimension, pattern sidewall angle, statistical deviations across large areas, and quantitative measures of pattern sidewall roughness. These efforts are driving toward the specification of a laboratory scale device capable of providing pattern dimensions during routine tests of fabrication processes.

\section{Ronald L. Jones and Wen-li Wu}

$\mathrm{T}$ he drive to reduce feature size to the sub-100 nm regime continues to challenge traditional metrology techniques for pattern characterization. Determination of the quality of the patterning process currently depends on the production of test patterns, such as line gratings, and evaluating dimensional control and the number of defects. As pattern sizes decrease, existing metrologies based on scanning electron microscopy and light scatterometry face significant technical hurdles in quantifying pattern dimensions and defects in dense, high-aspect ratio patterns used in modern semiconductor circuitry. There are currently no clear solutions to pattern quality measurement for future technology nodes with dimensions on the order of $30 \mathrm{~nm}$ and dimensional control to less than a nanometer.

To address these issues, NIST is developing a transmission x-ray scattering-based method capable of angstrom level precision in critical dimension evaluation over large, $(50 \times 50) \mu \mathrm{m}$, arrays of nanoscale periodic structures. In contrast to light scatterometry, Small Angle X-ray Scattering (SAXS) is performed in transmission using a sub-Angstrom wavelength. With a wavelength more than an order of magnitude smaller than the pattern size, the patterns can be analyzed using methods traditionally employed in crystallographic diffraction. The high energy of the x-ray beam allows the beam to pass through production quality silicon wafers without requiring a specialized sample environment (ultrahigh vacuum). While current measurements utilize the flexibility and wide array of instrumentation available at a synchotron source, initial results and the commercial availability of $\mathrm{x}$-ray sources and detectors suggests the technique is portable to a laboratory scale device.

As shown in the schematic below, the patterned sample is placed in an x-ray beam where the transmitted intensity is measured as a function of the scattering angle, $2 \theta$, on a $2-\mathrm{D}$ detector. The intensity is then fit to models describing the average pattern shape. To save valuable space within the total patterned area, industrial test patterns are typically smaller than $(50 \times 50) \mu \mathrm{m}$. Using a monochromator to define the wavelength and two sets of beam-defining slits, beam footprints of approximately $(40 \mathrm{x} 40) \mu \mathrm{m}$ have been successfully demonstrated. In contrast to 1-D techniques such as light scatterometry, the use of a 2-D detector allows the simultaneous characterization of all dimensions for patterns such as vias (arrays of cylinders standing perpendicular to the surface) and via pads (arrays of rectangles). In addition to measuring the pattern dimensions along the substrate plane, measurements at varying sample rotation angles, $\omega$, can be used to reconstruct the average 3-dimensional pattern shape.

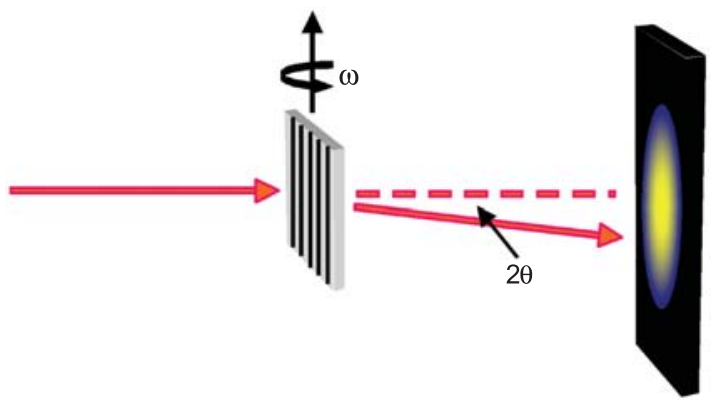

Figure 1: Schematic of SAXS geometry, showing incident and scattered beams (red lines), sample with pattern oriented at rotation angle $\omega$ and 2-D detector (right).

In collaboration with International SEMATECH, recent SAXS measurements of line gratings have demonstrated the capability of SAXS to measure fabricated patterns of thermally grown oxide with overall dimensions designed for current metrologies. The resulting detector image is a series of diffraction peaks along a single diffraction axis perpendicular to the orientation of the grating. The number of observable diffraction orders is in part determined by the pattern quality. The observation of over 30 orders of diffraction suggests the relatively high quality of these patterns and provides ample data for 
high-precision analysis. In addition, the number of observable diffraction orders serves as an immediate measure of pattern quality, termed a SAXS "fingerprint." With a measurement time on the order of a second, dose exposure matrices commonly used to evaluate optical imaging conditions can potentially be evaluated in a matter of minutes.

In addition to the SAXS "fingerprint," quantitative information is obtained through model fitting of the data. In contrast to reflection-based techniques, the models used here are relatively simple. Quantitative measures of the pattern repeat period and the line width are therefore obtained rapidly and precisely. For these samples, SAXS data have provided sub-nanometer precision in both average repeat distance and average line width. The precision of this measurement is in part dictated by the number of observable diffraction orders, which is in turn limited by the instrumental resolution and pattern quality. Ongoing studies will compare these results with measurements from light scatterometry, atomic force microscopy, and scanning electron microscopy.

In collaboration with the IBM T.J. Watson Research Center, the effects of specific types of defects in patterns on the resulting SAXS image are being explored. Shown in Figure 2, a simple model of an ideal line grating captures the main features of the diffraction spots; however, additional information
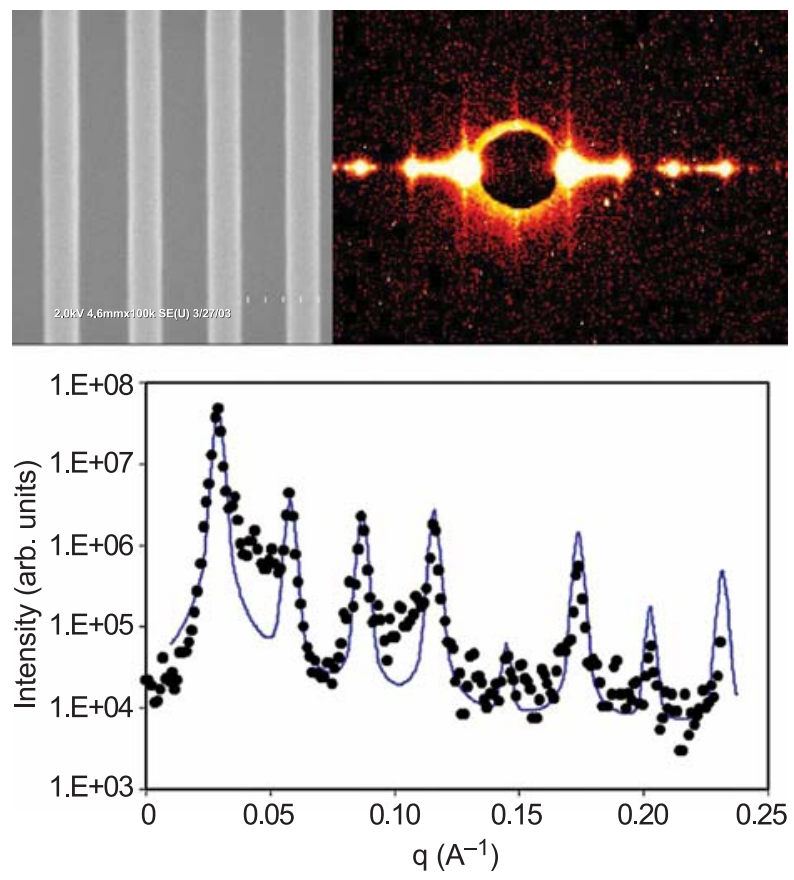

Figure 2: Top down SEM image (top left) of a photoresist grating on a silicon wafer compared to the resulting 2-D SAXS image (top right). Also shown are the intensities of the diffraction peaks as a function of scattering vector, $q(=4 \pi / \lambda \sin \theta$, where $\lambda$ is the $x$-ray wavelength) fit with a simple model (solid blue line). is observed in the 2-D image perpendicular to the diffraction axis. Streaks of intensity emanating from diffraction peaks are visible near the beamstop. These streaks are indicative of deviations from the ideal grating and may provide information about defects such as long wavelength line edge roughness.

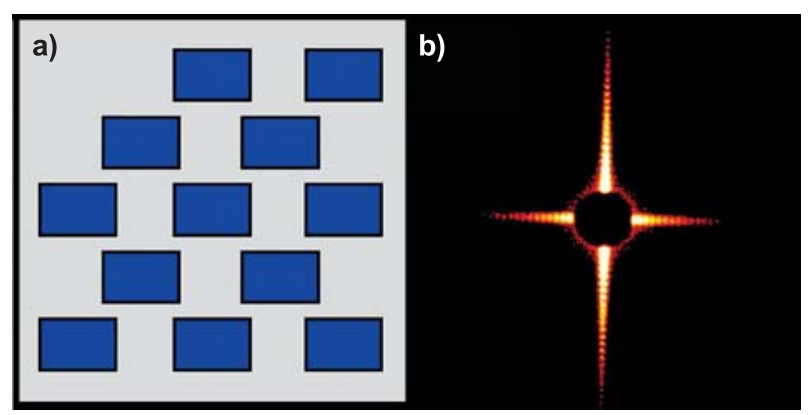

Figure 3: Schematic of via-pad patterns (a), where the blue rectangles represent etched regions in a low-k film, and (b) the resulting $S A X S$ detector image.

In addition to precisely measuring dense patterns of etched silicon oxide, the application of SAXS has been demonstrated for a wide range of materials that are currently used or are being explored for use in the microelectronics industry. We demonstrated the capabilities of SAXS on dense patterns of organic photoresist, silicon oxide, and nanoporous low-k samples (detector image from 2-D array of via pads shown in Figure 3). Additionally, measurements on samples of copper-filled, low-k patterns demonstrate the capability to probe multiple component and metallic patterns. Finally, the transmission geometry allows the probing of densely packed structures, whether buried or exposed, providing a potential to measure other nanoscale 3-D structures being explored for future microelectronic devices and photonic crystals.

Continuing efforts will focus on developing quantitative measures of line edge and sidewall roughness, isolating specific types of long-range order defects arising from masks, and reconstructing an average 3-D pattern shape. In addition, further investigations will provide precise specifications for a laboratory scale device capable of similar measurements.

\section{For More Information on this Topic}

R. Jones, T. Hu, W. Wu, E. Lin (Polymers Division, NIST); G. Orji, T. Vorburger (Precision Engineering Division, NIST); A. Mahorowala (T.J. Watson Research Center, IBM); G. Barclay (Shipley Co.); D. Casa (Advanced Photon Source, Argonne National Laborotory)

R.L. Jones, T.J. Hu, E.K. Lin, W.-L. Wu, D.M. Casa, N.G. Orji, T.V. Vorburger, P.J. Bolton, G.G. Barclay, Proc. SPIE 5038, 191 (2003). 


\section{High-throughput Measurements of Elastic Moduli of Polymer Thin Films}

As technology continues to strive towards smaller, thinner, and lighter devices, more stringent demands are being placed on polymer films as diffusion barriers, dielectric coatings, electronic packaging, etc. The material properties of thin films can be drastically different from that of the bulk material. Therefore, there is a growing need for testing platforms that allow for rapid determination of the mechanical properties of thin polymer films/coatings. We demonstrate here a novel measurement technique that yields the elastic modulus of supported polymer films in a rapid and quantitative manner without the need for expensive equipment or material-specific modeling.

\section{Christopher M. Stafford}

$\mathrm{T}$ here exist few techniques to measure the mechanical properties of polymer thin films (e.g., nanoindentation, atomic force microscopy (AFM), surface acoustical wave spectroscopy, and brillouin light scattering), each having their own limitations and none of which are positioned to be applied as high-throughput measurements. We present a reliable, high-throughput technique by which the elastic modulus of thin polymer films can be measured both rapidly and quantitatively. Indeed, this technique is very simple and practically any laboratory, academic or industrial, can perform such measurements with only modest investment in equipment.

This measurement platform exploits a classic mechanical instability that occurs in laminates and sandwich structures under compression. In this geometry, a highly periodic buckling instability arises from a mismatch of the elastic moduli of a relatively stiff polymer coating that has been applied to a soft polydimethylsiloxane (PDMS) substrate. The wavelength of the wrinkles can be measured rapidly by conventional light scattering. In addition, the sample can be rastered across the beam to map out the properties of the entire film if the sample is comprised of a gradient library, or if the sample is uniform, a multitude of images can be acquired to generate sufficient statistics. We have denoted this technique as Strain Induced Elastomer Buckling Instability for Mechanical Measurements (SIEBIMM). Since this is an intrinsically local measurement, this technique is well suited for measurements of combinatorial libraries with spatially varying properties that can be prepared by existing methodologies developed at NIST.

In general, thin polymer films are prepared on silicon substrates either by flow coating or spin coating. The thickness $(h)$ of each film is measured extensively by conventional interferometry. Films are transferred to PDMS strips via aqueous immersion to produce a laminate structure. Upon application of strain, the disparity in the Poisson's ratios $(v)$ between the PDMS substrate and the polymer film results in a net compressive stress on the glassy film perpendicular to the strain direction. Conversely, higher degrees of compression can be obtained by pre-stretching the PDMS prior to transfer of the polymer film and then releasing the stressed PDMS/film laminate. One mechanism by which the film can relieve this applied stress is to buckle, thus producing a sinusoidal phase grating whose periodicity or wavelength $(d)$ can be rapidly quantified by conventional light scattering.

The modulus of the upper film is calculated by the following equation (as detailed by H.G. Allen, Analysis and Design of Structural Sandwich Panels):

$$
E_{p}=12 E_{m}\left[\left(3-v_{m}\right)\left(1+v_{m}\right)\right]^{-1}[q h]^{-3}
$$

where $E_{p}$ is the modulus of the upper film, and $E_{m}$ and $v_{m}$ are the modulus and Poisson's ratio of the PDMS substrate, respectively. The Poisson's ratio for PDMS was measured to be $0.50 \pm 0.05$. In this formalism, $q=2 \pi / d$ where $d$ is the periodicity of the wrinkles. We find that this equation is valid for $E_{p} / E_{m}>20$, which allows a wide variety of materials to be measured.

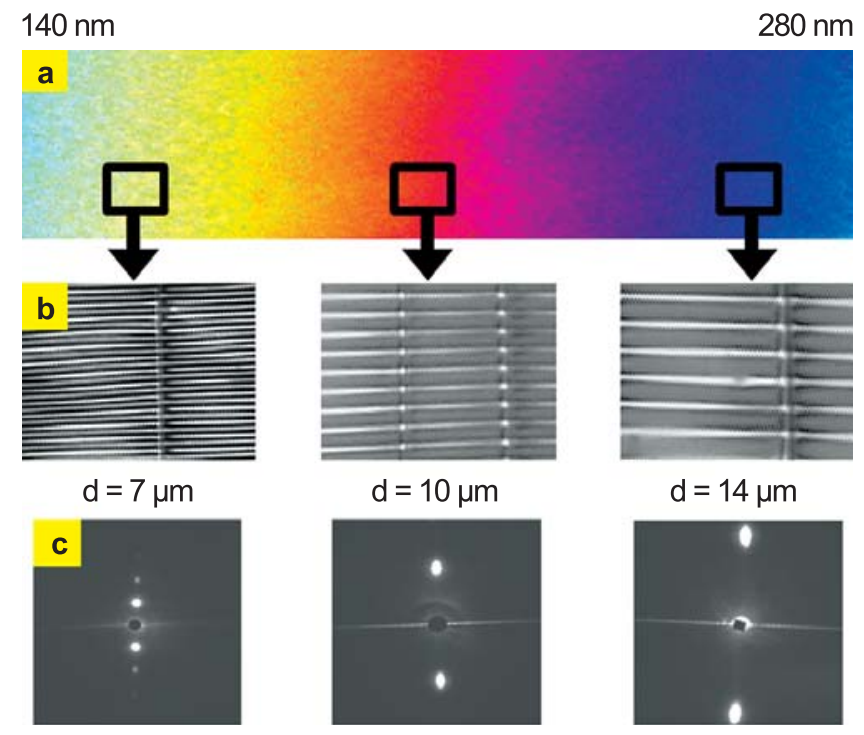

Figure 1: (a) Optical image of a thickness gradient of polystyrene, (b) optical images of the buckling pattern as a function of film thickness, (c) light diffraction patterns from the periodic wrinkles shown in (b). 
Initial validation of this concept focused on a thickness gradient of polystyrene ranging from $140 \mathrm{~nm}$ to $280 \mathrm{~nm}$ in thickness. According to Equation 1, the buckling wavelength should be inversely proportional to the thickness of the upper film for a film of constant modulus, $E_{p}$. Figure 1 demonstrates the validity of Equation 1: as the film thickness increases by a factor of two, the buckling wavelength also increases by a factor of two as measured by both optical microscopy and light scattering. Through Equation 1, the modulus of the polystyrene film is calculated to be $3.17 \mathrm{GPa} \pm 0.11 \mathrm{GPa}$ for the entire range of thickness. These values are in excellent agreement with the measured bulk value of $3.22 \mathrm{GPa} \pm 0.05 \mathrm{GPa}$ for the same polymer.

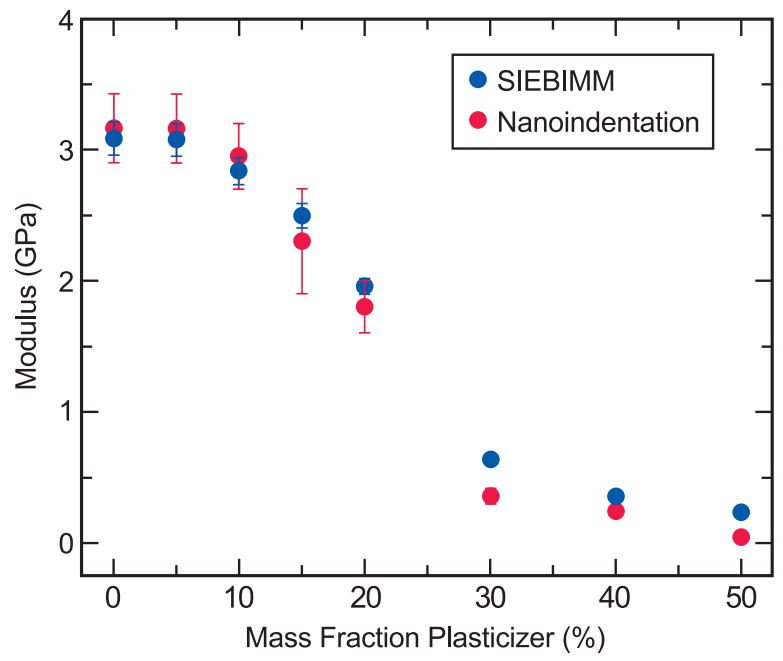

Figure 2: Modulus of polystyrene thin films as a function of mass fraction of added plasticizer, dioctyl phthalate.

This method was also applied to a model system that varied in mechanical properties. Here, a common plasticizer (dioctyl phthalate) was solution blended with polystyrene prior to spin coating. As shown in Figure 2, the measured modulus (blue) of the polystyrene film decreases in a sigmoidal manner with increasing concentration of added plasticizer. These results were compared against nanoindentation data (red) obtained on identical films supported on silicon wafers. The agreement between the buckling method and nanoindentation is markedly good.

Measurements were also performed on blends of polystyrene-polyisoprene-polystyrene (P(S-I-S)) triblock copolymers to demostrate the applicability to soft, structured materials. Two commercially available copolymers of $\mathrm{P}(\mathrm{S}-\mathrm{I}-\mathrm{S})$ with different fractions of the glassy component (PS) were solution blended prior to spin-coating into micron thick films. Since the periodicity of block copolymer microdomains is on the order of $30 \mathrm{~nm}$, thick films should exhibit bulk-like mechanical behavior. Also, the microphase-separated structure of the copolymer should not interfere with the light scattering experiment due to the small domain sizes. The results of this experiment can be seen in Figure 3 where thin film data are shown in blue and bulk tensile tests are shown in red. While the bulk tests suffer from a great deal of scatter, perhaps due to variations in sample preparation and handling, buckling measurements show a comparatively smooth increase in modulus with increasing PS fraction. As shown in Figures 2 and 3, the moduli range accessible by this method is as low as $5 \mathrm{MPa}$ and as high as several GPa.

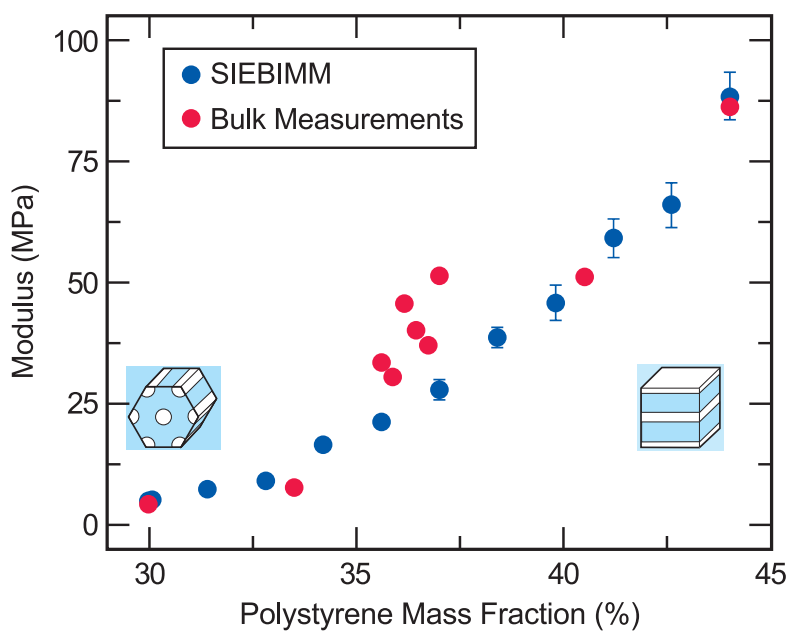

Figure 3: Modulus of $P(S-I-S)$ triblock copolymer blends with an increasing glassy component, $P S$.

We have developed a novel, high-throughput technique that allows the elastic modulus of polymer thin films to be determined rapidly and quantitatively. The SIEBIMM technique exploits a strain-induced buckling instability in elastomer-supported films that exhibits a modulus dependent wavelength. Conventional light scattering can quickly access the wavelength of the wrinkles, and the modulus can be calculated using a simple model. We measured films with thicknesses ranging from several micrometers to $20 \mathrm{~nm}$ and films with a range of moduli from several GPa to $5 \mathrm{MPa}$.

Research is continuing in the areas of nanoporous low- $k$ films, metal and ceramic thin films, and UV-curable materials. In addition, the method shows promise for the study of such issues as aging effects, creep, stress-relaxation, and viscoelasticity. There is also interest in exploiting these sinusoidal surfaces as tunable phase gratings and using these tunable textured surfaces to examine a number of scientific problems, such as patterned cell growth and anisotropic adhesion.

\section{For More Information on this Topic}

C.M. Stafford, A. Karim, E.J. Amis (Polymers Division, NIST), C. Harrison (Schlumberger-Doll Research) 


\section{Elastic Flow Instability in Polymer-Dispersed Carbon Nanotubes}

\begin{abstract}
Novel composites engineered from polymers and carbon nanotubes offer the promise of plastics with enhanced thermal, electronic, and mechanical properties, but the ability to control and quantify particle dispersion in such materials is an unresolved issue of fundamental importance. Of particular interest is how processing flows influence tube dispersion and orientation. We are developing metrologies and methods that directly address the fundamental nature of elastic flow instabilities in polymer-dispersed carbon nanotubes, enabling better control of dispersion during flow processing of melts and suspensions.
\end{abstract}

\section{Erik K. Hobbie}

$\mathrm{T}_{\mathrm{s}}^{\mathrm{h}}$ he clustering of small spherical particles has been studied extensively in a broad range of systems. In contrast, relatively little is known about the flocculation of asymmetrical particles, such as platelets, rods, or fibers. Given the current interest in dispersing anisotropic nanoparticles in organic materials to enhance physical properties, a deeper understanding seems warranted. An almost universal aspect of aggregation is inter-particle attraction, but external hydrodynamic forces, often used to disperse such particles, can alone induce clustering in highly anisotropic suspensions, creating unique challenges and issues relating to the flow processing of such materials.

We recently observed an elastic instability associated with flow-induced clustering in polymer dispersed carbon nanotubes. Kinetic measurements under varied confinements and shear stresses were compared with simulations of flocculation in flowing fiber suspensions, and we found an intriguing rheological signature consistent with highly elastic domains. Small-amplitude oscillatory shear measurements reveal this extraordinary elasticity, with homogenized semi-dilute suspensions showing gel-like behavior at long time scales. The data suggest that the underlying instability may in fact be universal to a number of flowing complex fluids, with important implications for the flow processing of polymer-carbon nanotube composites.

Multi-walled carbon nanotubes (MWNTs) were grown via chemical vapor deposition. A typical electron micrograph is shown in the inset to Figure 1(a). Based on such measurements, the mean diameter is $d \approx 50 \mathrm{~nm}$. Due to their length and optical contrast, individual MWNTs are discernable in optical micrographs of $25 \mathrm{x}$ or higher, and from 200x images of thin-film dispersions, the mean length is $L \approx 12 \mu \mathrm{m}$. The suspending polyisobutylene fluid (PIB) is Newtonian over a broad range of shear rates, with a shear viscosity of $10 \mathrm{~Pa}-\mathrm{s}$ at $25{ }^{\circ} \mathrm{C}$. Dispersions are prepared by dissolving the PIB in sonicated MWNT-toluene suspensions, which are stirred continuously as the solvent is removed. Suspensions of primary interest contain $1.7 \times 10^{-3}$ mass-fraction MWNT in PIB and are semi-dilute, with $\mathrm{nL}^{3} \approx 45$ and $\mathrm{nL}^{2} \mathrm{~d} \approx 0.19$, where $n$ is the number of tubes per unit volume. The tubes in PIB are non-sedimenting over the time scales in question.
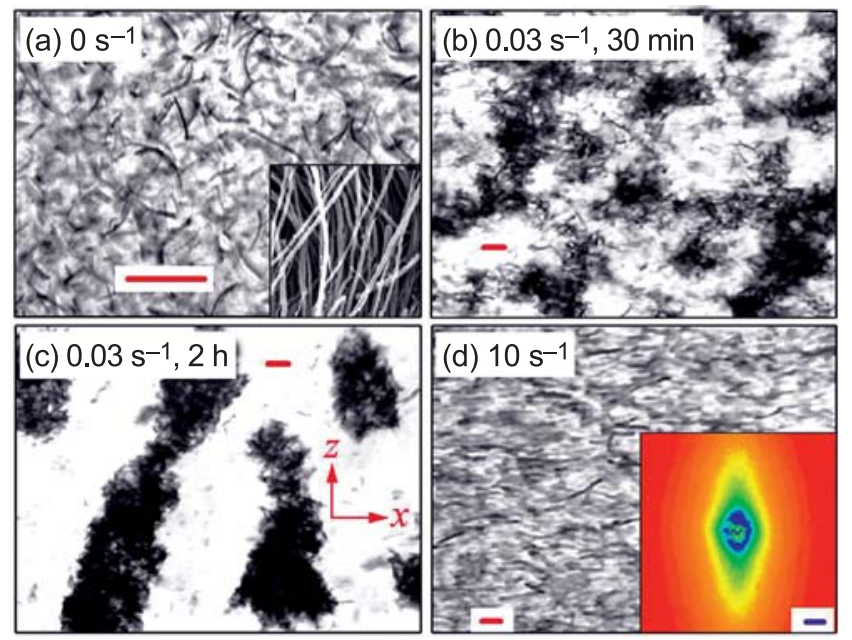

Figure 1: (a) Optical micrograph of MWNTs in a quiescent PIB dispersion. The inset (width $=1.3 \mu \mathrm{m}$ ) is a SEM image of the $M W N T$ s before dispersion in PIB. (b) Optical micrograph $30 \mathrm{~min}$ after quenching the mixture to a shear rate of $\dot{\gamma}_{o}=0.03 \mathrm{~s}^{-1}$. The flow $(x)$ direction is horizontal. (c) Optical micrograph $2 \mathrm{~h}$ after the quench. (d) Optical micrograph 15 min after quenching the sample in (c) to $\dot{\gamma}_{m}=10 \mathrm{~s}^{-1}$.

Optical microscopy ( $5 \mathrm{x}$ to $25 \mathrm{x}$ ) is used to observe the motion of MWNTs and the formation of aggregates under shear, with flow along the $x$-axis, a constant velocity gradient along the $y$-axis, and vorticity along the $z$-axis. Measurements are taken in the $x$ - $z$ plane. The sample is confined between two parallel quartz plates separated by a variable gap, $h$. The upper plate rotates at an angular speed that sets the shear rate, $\dot{\gamma}=\partial v_{x} / \partial y$, at a fixed point of observation in the middle of the plates. A controlled-strain rheometer in cone-and-plate and parallel-plate configurations provides steady and oscillatory shear measurements of the rheology. All measurements were performed at $25^{\circ} \mathrm{C}$.

The aggregates have "melted" and the redispersed tubes broadly orient with the direction of flow, as evident in the light-scattering pattern (inset Figure 1(d), scale bar $\left.=1 \mu \mathrm{m}^{-1}\right)$. The scale bar is $10 \mu \mathrm{m}$ and the gap (h) is $70 \mu \mathrm{m}$. 
Under weak shear, the tubes form macroscopic domains consisting of diffuse MWNT networks. Figure 1 shows optical micrographs of (a) a quiescent dispersion, (b \& c) aggregation at $\dot{\gamma}_{o}=0.03 \mathrm{~s}^{-1}$, and (d) redispersed MWNTs after dissolution at $\dot{\gamma}_{m}=10 \mathrm{~s}^{-1}$, where light scattering reveals a steady-state distribution of orientations broadly peaked around $\hat{\mathbf{x}}$. In simple shear, the long axis of an isolated $\operatorname{rod}$ rotates around $\hat{\mathbf{z}}$ with a period, $\tau$, that scales as $\dot{\gamma}^{-1}$. In semi-dilute suspensions, hydrodynamic interactions determine the distribution of such orbits. The Peclet number is less than $10^{-4}$, implying that hydrodynamic forces overwhelm Brownian forces.

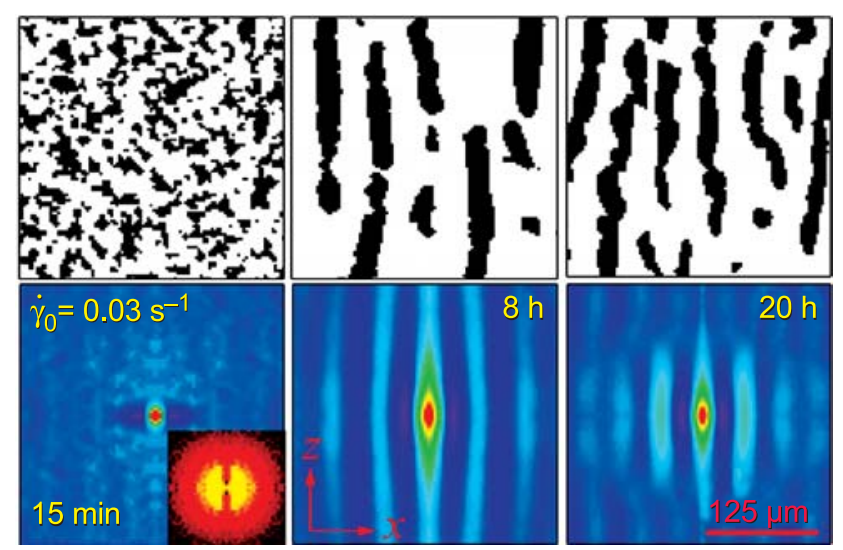

Figure 2: Evolution of a confined dispersion (upper), where the micrographs have been converted to binary images. The lower images are the corresponding $c(\boldsymbol{r})$. The flow direction is horizontal, the vorticity axis is vertical, and the red scale bar $(125 \mu \mathrm{m})$ applies to all six images. As in Figure 2, $h=70 \mu \mathrm{m}$. The inset to the lower left image is its FFT (width $=1.2 \mu \mathrm{m}^{-1}$ ), which gives the ubiquitous "butterfly" pattern.

By varying $h$, we observe the transition from bulk to confined growth. To quantify the latter, we convert micrographs into binary images in which the clusters are black and the surrounding fluid white (upper images, Figure 2), defining a coarse composition field $\psi(\mathbf{r})$. Ensembles at each annealing time, $t$, are used to compute the two-point correlation function, $c(\mathbf{r})=\langle\psi(\mathbf{r}) \psi(0)\rangle$ (lower images, Figure 2), and the steady-state morphology diagram in the $h-\dot{\gamma}$ plane is shown in Figure 3. In region A, moderate aspect ratio domains exhibit broad vorticity alignment. In region $\mathrm{S}$, these domains coarsen along $\hat{\mathbf{z}}$ into macroscopic striped patterns. The dashed curve marks a region of "metastability" in which the striped pattern is transient.

Recent simulations of flow-induced flocculation in non-Brownian fiber suspensions suggest that interparticle friction leads to aggregation in the absence of attractive interactions, particularly at low-shear stress and high-fiber stiffness. The MWNT bending modulus can be compared with values for typical organic fibers, as can the shape of the inter-particle potential and coefficient of friction. Our measurements are in agreement with simulation. With an elastic bending modulus $10^{3}$ times larger than that of a typical fiber, the MWNTs are flexible enough to exhibit shape deformation, yet they readily entangle and interlock to form large coherent structures under weak shear. Held together by elastic forces, the diffuse clusters store sizeable energy, which we infer from linear viscoelastic measurements of the storage modulus, $G^{\prime}(\omega)$, as a function of $n$. Other unusual features, such as a negative first normal stress difference, are currently under in-depth investigation.

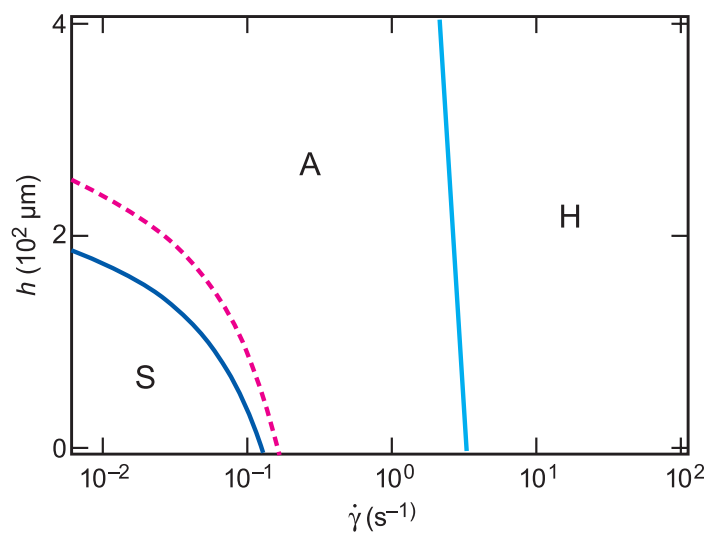

Figure 3: The measured late-t "pattern diagram" in the $h-\dot{\gamma}$ plane for the semi-dilute, non-Brownian MWNT-PIB suspensions of interest, with homogeneous (H), aggregated (A), and "striped" (S) regions as indicated.

We have observed the early-time domain pattern [Figure 2 (left), weak minima in $c(x)$ ] in a host of flowing complex fluids, including polymer blends, semi-dilute polymer solutions, physical polymer-clay gels, and thixotropic clay gels, all of which fall within the simple paradigm of weakly interacting elastic domains suspended in a less viscous fluid. The homogenized semi-dilute dispersions also exhibit solid-like behavior at long timescales, suggesting the presence of a weak "network" or gel, further reminiscent of other systems that exhibit this type of flow-induced macrostructure. The role of confinement in the growth of periodic structures in these systems is intriguing and merits detailed computational consideration. We expect that these issues will have direct consequences for the processing of nanocomposite melts, and we are currently extending these measurements to single-walled carbon nanotubes.

\section{For More Information on this Topic}

E.K. Hobbie, S. Lin-Gibson, J. Pathak (Polymers Division, NIST); H. Wang (Michigan Tech); E. Grulke (University of Kentucky) 


\section{Scaffold Structure and Cell Function through Multi-Modal Imaging and Quantitative Visualization}

\begin{abstract}
Non-destructive, in vitro evaluation of tissue engineered medical products (TEMPs) will shorten their development time. Imaging techniques such as collinear optical coherence and confocal fluorescence microscopies are being used to address the challenges of imaging these systems. An equally important component of the work is the image quantification as a vehicle to evaluate the voluminous imaging data.
\end{abstract}

\section{Joy P. Dunkers and Marcus T. Cicerone}

$\mathrm{T}$ issue engineered medical products (TEMPs) are often three-dimensional (3D) hybrid materials consisting of a porous scaffold upon which the tissue is grown. While it is generally understood that a complex interaction of many variables influences the success of the TEMPs, the precise nature of these interactions has yet to be worked out in many instances. A significant difficulty in furthering the understanding of the interaction between these factors and cell behavior is the lack of a high-resolution imaging technique that can penetrate deeply and non-destructively into the scaffold. We have developed an approach that uses advanced optical imaging to non-invasively monitor the developing tissue. However, before any assessment of the tissue viability can be made, the volumes of imaging data must be rigorously analyzed. Therefore, an equally important component of this effort is image visualization and quantification. Progress in these areas is summarized below.

\section{Multi-Modal Imaging}

We have constructed an instrument that can gather information on a TEMP using multiple imaging modalities. This means that each channel of imaging data provides different but complimentary information. Optical coherence microscopy (OCM) was chosen as the technique to image scaffold, cell, and tissue structure because of its unique combination of high resolution $(\approx 1 \mu \mathrm{m})$ and high sensitivity $(>100 \mathrm{~dB})$. $\mathrm{OCM}$ is an interferometric technique that uses both confocal and coherence gating mechanisms for stray light rejection, rendering it comparable in resolution to laser scanning confocal microscopy but far superior in imaging depth. We have added confocal fluorescence microscopy (CFM) to the OCM to collect information on cell function using traditional cell staining techniques. In our collinear instrument, we collect volumetric images of cell and scaffold structure using the OCM channel and cell function using the CFM channel. Each channel is then overlayed in the rendered image for maximal insight.

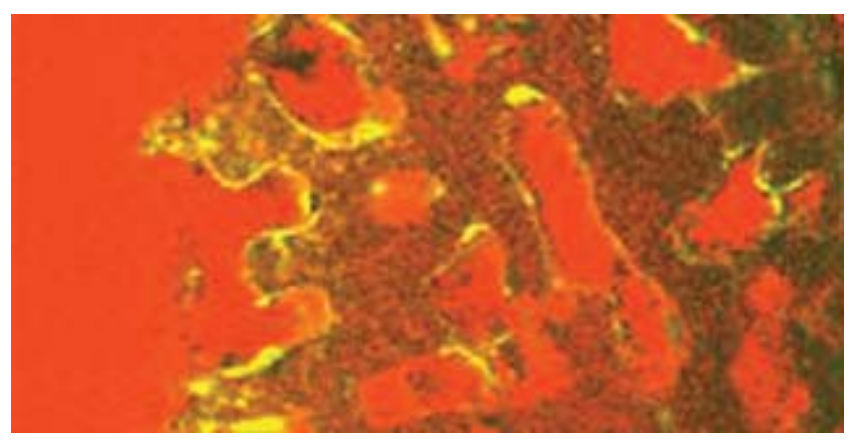

Figure 1: Image of merged and registered OCM and CFM images of the cultured PCL scaffold at $145 \mu \mathrm{m}$ below the surface.

Figure 1 displays merged and registered OCM and CFM images $145 \mu \mathrm{m}$ below the surface of a TEMP. The TEMP consists of a volume fraction of $50 \%$ poly( $\varepsilon$-caprolactone) (PCL) scaffold that was cultured with fetal chick osteoblasts for 10 weeks and stained with a nuclear stain. In Figure 1, the regions of low OCM signal are red (pores), high OCM signal are black (scaffold), and regions of high CFM signal are yellow (cells). CFM complements OCM by allowing us to positively identify stained tissues at more shallow depths. Once identified, OCM allows us to discriminate these tissues from scaffold and, thus, view them at a greater depth. This will form the basis of structure-property relationships for TEMPs based on microscopic characterization of scaffold properties and concomitant cellular responses.

\section{Quantitative Visualization}

Quantification of scaffold properties must be performed to establish scaffold structure and cell function relationships and to optimize scaffold design. One of our goals is to design an approach that is valid with any pore structure. Figure 2 displays a volumetric view of the PCL scaffold images collected using x-ray computed tomography. In this figure, the pores are colored in red and the scaffold in green. From viewing this image, one gets a qualitative sense of the need for such an approach. Heterogeneity in the microstructure is exhibited by the difference in pore size, shape, and anisotropy as seen on the different faces of the volume. Pore volume, size distribution, tortuosity, and connectivity are metrics of interest for the scaffold microstructure. 
An example of the type of information gleaned from the imaging data is shown below. Figure 3 displays the pore size as measured by the chord length distribution function (CLDF). The CLDF is the probability of finding a chord of length $l$ between $x$ and $x+d x$ entirely in one phase. Chords are defined as the segments formed by the intersection of lines with the interface between two phases.

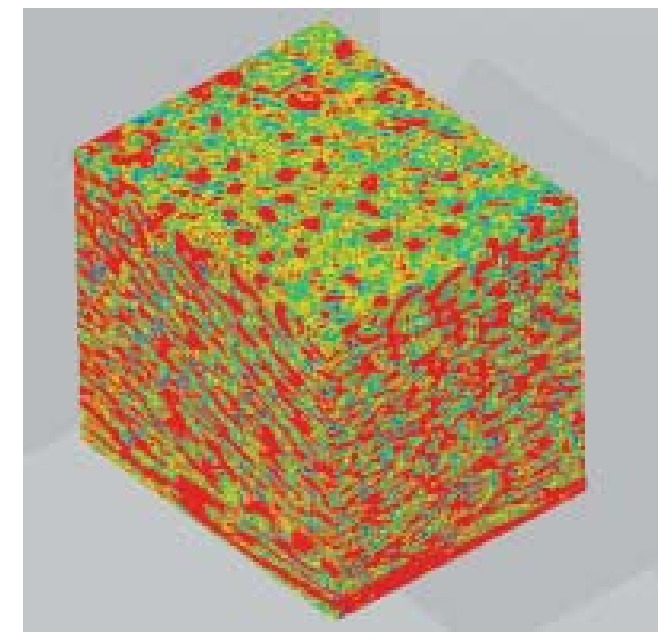

Figure 2: X-ray computed tomographic reconstruction of a PCL based scaffold. Dimensions: $1.0 \mathrm{~mm}$ on each side.

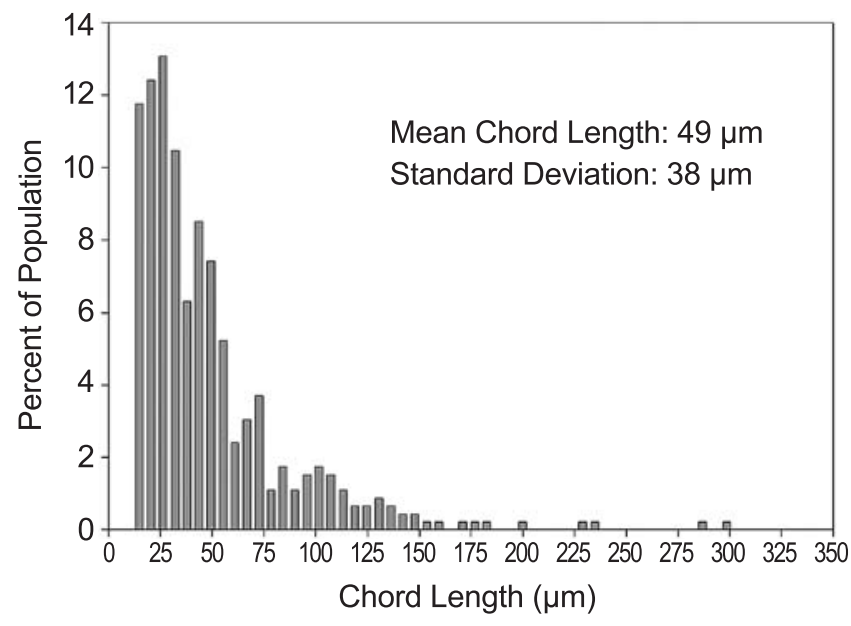

Figure 3: Example of a CLDF from one image plane in Figure 2.

Another important aspect of the effort to optimize scaffold design is the need to balance competing requirements. High porosity is required because of the need for cell migration, proliferation, and nutrient influx. However, the drive towards higher and higher pore volumes opposes the need for certain mechanical property requirements, especially in orthopedic applications. To this end, we are using 3D finite element analysis (FEA) to develop an analytical tool to predict the relationship between the effective properties and individual constituent properties of TEMPs based on real material images. This relationship, plus the analysis of the structural problem of interest, provides a means of optimizing the performance of TEMPs by varying individual constituent properties without conducting a variety of time-consuming experiments. Initially, the properties of interest are anisotropic elastic constants. Figure 4 displays a typical mesh of FEA based on a section of the PCL scaffold from Figure 2.

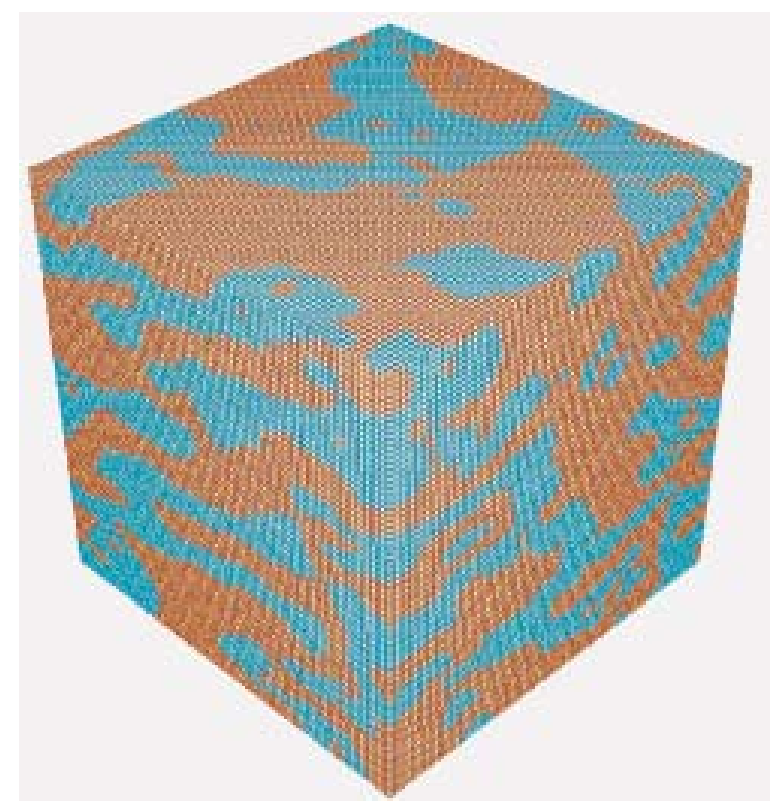

Figure 4: Finite element mesh of a subsection of scaffold shown in Figure 2. The dimension is $274 \mu \mathrm{m}$ on each side.

This work represents a systematic, integrated approach to the study of structure/function relationships and optimal design in TEMPs. We will be able to extract metrics for the anisotropic scaffold microstructure and properties and use that information to understand their influence on cell function, and, on a larger scale, TEMP viability.

\section{For More Information on this Topic}

\section{F. Landis, M. Chiang (Polymers Division, NIST)}

M.T. Cicerone, J.P. Dunkers, and N.R. Washburn, "Towards in-situ Monitoring of Cell Growth in Tissue Engineering Scaffolds: High Resolution Optical Techniques," Tissue Engineered Medical Products (TEMPS) ASTM STP, 1452, November 3, 2002, Miami Beach, FL.

For information involving chemical imaging of TEMPs, see the project page entitled "Coherent AntiStokes Raman Micro-spectroscopy ( $\mu$-CARS) for Understanding Tissue Growth in Scaffold Constructs" by M. Cicerone and T. Kee. 


\section{Macromolecular Dynamics via Inelastic Neutron Scattering}

\begin{abstract}
Neutron scattering has been an invaluable tool for the advancement of basic polymer science. At NIST, we are uniquely situated to leverage the world-class inelastic neutron scattering facilities being developed at the NIST Center for Neutron Research (NCNR). Our mission is to develop fundamental measurements and uses of these resources in a manner that enables critical and emerging technologies.
\end{abstract}

\section{Christopher L. Soles}

$\mathrm{W}$ e illustrate two examples where fundamental studies of macromolecular dynamics, via neutron scattering, have spawned metrologies that are critical for an emerging technology. These include the impact of thin-film dynamics on chemically amplified photoresists and protein preservation. In each instance, the insight from neutron scattering has provided invaluable insight into an industrially relevant and difficult problem.

The semiconductor industry is faced with a relentless drive to produce devices with smaller dimensions. This dictates that lithographers use increasingly thinner photoresist films, soon to be less than $100 \mathrm{~nm}$ thick. This is of concern since properties can deviate when polymer films approach these dimensions. Industrial partners indicate that resist performance typically deteriorates in films less than $100 \mathrm{~nm}$ thick. Detailed concerns focus on dynamics because the resolution of a chemically amplified photoresist requires a delicate balance of photoacid $\left(\mathrm{H}^{+}\right)$ reaction and diffusion; it is reasonable to anticipate that diffusion is coupled to the dynamics in the film.

To directly probe the dynamics of thin polymer films, we designed incoherent neutron scattering measurements utilizing the NCNR's High Flux Backscattering Spectrometer (HFBS), which characterizes atomic vibrations and relaxations on time scales $\geq 200 \mathrm{MHz}$ (faster than a few nanoseconds) in terms of a Debye-Waller factor and the mean-square atomic displacement $\left\langle u^{2}\right\rangle$. In several publications, ${ }^{[1]}$ we observe a strong suppression of $<u^{2}>$ with decreasing thickness, even in films as thin as $75 \AA$.

To determine if reduced polymer mobility is germane to the observed deviations in thin film lithography, the diffusion and reaction of $\mathrm{H}^{+}$into a photoresist polymer (PBOCSt) was studied through a series of bilayer diffusion couples. ${ }^{[2]}$ This revealed a strong decrease of the effective reaction-diffusion coefficient when the films were less than $100 \mathrm{~nm}$. HFBS measurements on these PBOCSt films confirm a suppression of $<\mathrm{u}^{2}>$ at a similar film thickness. ${ }^{[3]}$ We then use a relation between $<u^{2}>$ and diffusivity and predict the film thickness at which the $\mathrm{H}^{+}$reaction-diffusion kinetics diverge. ${ }^{[3]}$ Figure 1 displays this correlation in conjunction with moisture diffusion coefficients obtained with a quartz crystal microbalance. ${ }^{[4]}$ That the diffusivity of a non-reactive water molecule tracks the $\mathrm{H}^{+}$reaction-diffusion suggests that deviations with PBOCSt film thickness reflect changes in the transport properties, not the chemical kinetics.

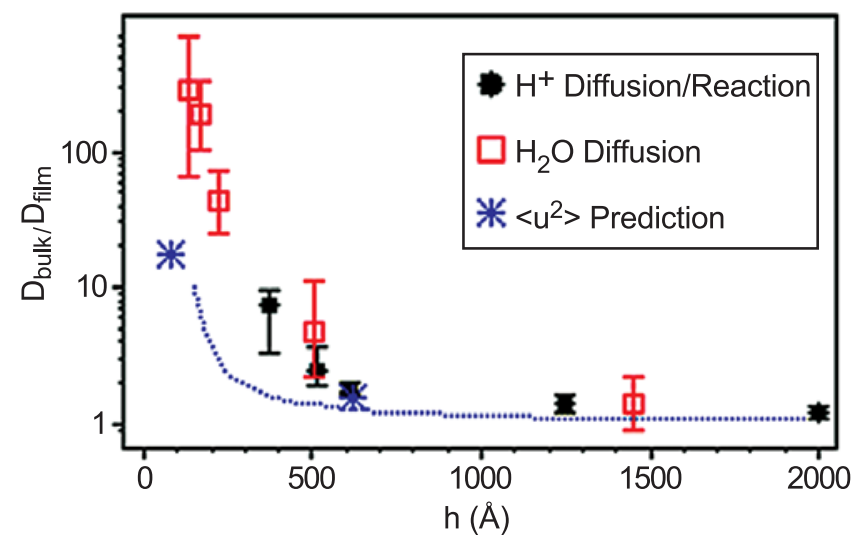

Figure 1: Reduced mobility/diffusivity as a function of PBOCSt film thickness directly reflected by $\mathrm{H}^{+}$(circles) and $\mathrm{H}_{2} \mathrm{O}$ (squares) motions and predicted by $<u^{2}>$ (stars \& dotted line).

The impact of this work is clear. The resist community was not aware that diffusive properties could change with film thickness. Dr. Robert Brainard of the Shipley Corporation comments, "I really think this work is an eye opener." We also have evidence indicating that this reduced diffusivity in thin-resist films also leads to slower dissolution rate in the aqueous base developer.

Switching to preservation, proteins are often lyophilized (freeze-dried) from viscous sugar solutions to stabilize biochemical functionality. The first requirement of a preservation media is that it does not denature the protein due to thermodynamic interactions. Screening materials for this incompatibility is fast and easy. The difficulty comes when one must predict which of the thermodynamically compatible glasses will be the most effective preservation media. This requires tedious long-term stability studies, which rely upon trial and error. Dr. Robert Langer, Chair of the FDA's Science Committee, identified the need to develop protein stabilization technologies as one of the most pressing problems facing pharmaceutical and biomaterials sectors. 


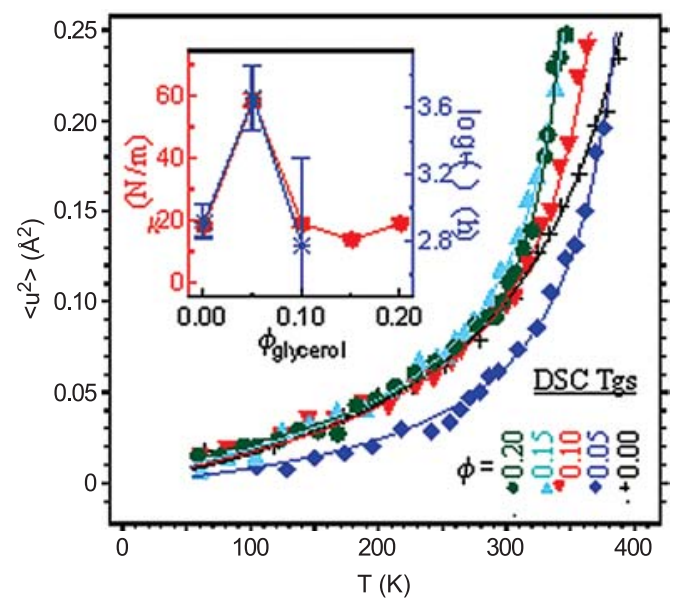

Figure 2: The T dependence of $\left\langle u^{2}>\right.$ with glycerol content. The inset correlates the vibrational spring constant $k$ between (40 and 200) $K$ with the protein lifetimes $\tau$; both $k$ and the degree of protein stability show a pronounced maximum at $\phi_{\text {glycerol }}=0.05$.

We demonstrate, by coupling $\mathrm{HFBS}^{[5]}$ and protein activity measurements, ${ }^{[6]}$ that $<u^{2}>$ indicates the efficacy of a glass at stabilizing a protein. Figure 2 shows the thermal evolution of $\left\langle u^{2}>\right.$ in a series of trehalose glasses diluted with mass fractions of glycerol $\left(\phi_{\text {glycerol }}\right)$ at $0,0.05,0.10,0.15$, and 0.20 . Increasing $\phi_{\text {glycerol }}$ monotonically decreases the calorimetric glass transition temperature $T_{\mathrm{g}}$, indicated by the upturn of $<u^{2}>$.

However, a maximum suppression of $\left\langle u^{2}\right\rangle$ occurs at $\phi_{\text {glycerol }}=0.05$, indicating that small amounts of glycerol are best for suppressing the atomic fluctuations in trehalose. This suppression of $\left\langle u^{2}\right\rangle$ is quantified between (40 and 200) $\mathrm{K}$ in terms of a harmonic oscillator. The elastic spring constant $k$ (inset Figure 2) of these vibrations shows a pronounced maximum at $\phi_{\text {glycerol }}=0.05$.

Figure 2 (inset) also displays the deactivation lifetimes $(\tau)$ for horseradish peroxidase lyophilized in these trehalose mixtures. An order of magnitude increase in protein stabilization at $\phi_{\text {glycerol }}=0.05$ coincides with the peak in $k$. While the data are not shown, the same trend is seen with the protein alchohol dehyrodgenase. In short, a strong suppression of $\left\langle u^{2}\right\rangle$ indicates a highly effective preservation media. This insight from neutron scattering enables a powerful metrology for screening candidate materials. To further promote this tool, we are simultaneously developing Raman scattering (optical) methodologies that provide comparable information without the need of a nuclear reactor. ${ }^{[7]}$

It is not surprising that suppressing the nanosecond atomic fluctuations leads to enhanced stability. These local motions are necessary precursors for the global conformational fluctuations in a protein that occur on the physiological time and length scales. Suppressing $<u^{2}>$ makes protein unfolding or denaturation more difficult. Likewise, deactivation can also be induced by the diffusion of reactive gases into the protein through the glassy matrix. Figure 1 clearly illustrates that suppressing $<u^{2}>$ retards diffusion.

Molecular insight into the suppression of $<u^{2}>$ can be inferred by positron annihilation lifetime spectroscopy, which quantifies unoccupied volume at the interatomic or nanometer length scales. Glasses with open structures at these levels also display enhanced dynamical fluctuations ${ }^{[8]}$ (evidenced via $\left\langle u^{2}>\right.$ ) and softened collected atomic vibrations. ${ }^{[9]}$ This suggests that a small amount of glycerol (or thin-film confinement in Figure 1) enhances intermolecular packing. This stiffening or "caging" decreases the success rate for vibrations turning into relaxations. It is these relaxations that lead to diffusion or protein deactivation.

\section{References}

1. C. Soles, J. Douglas, W. Wu, Phys. Rev. Lett. 88, 037401 (2002); ibid, Macromolecules 36, 373 (2003); C. Soles, E. Lin, J. Lenhart, R. Jones, W. Wu, D. Goldfarb, M. Angelopoulos, J. Vac. Sci. Technol. B 19, 2690 (2001).

2. D. Goldfarb, M. Angelopolous, E. Lin, R. Jones, C. Soles, J. Lenhart, W. Wu, J. Vac. Sci. Technol. B 19, 2699 (2001).

3. C. Soles, J. Douglas, E. Lin, J. Lenhart, R. Jones, W. Wu, D. Goldfarb, M. Angelopolous, J. Appl. Phys. 93, 1978 (2003).

4. C. Soles, R. Jones, J. Lenhart, V. Prabhu, W. Wu, E. Lin, D. Goldfarb, M. Angelopolous, Proc. SPIE 5039, 336 (2003).

5. M. Cicerone and C. Soles, Biophys. J. (2003) (submitted).

6. M. Cicerone, A. Tellington, L. Trost, A. Sokolov, Bioprocess Intl. 1, 23 (2003).

7. G. Caliskan, A. Kisliuk, A. Tsai, C. Soles, A. Sokolov, J. Chem. Phys. 118 (2003); 4230; ibid, J. Non-Cryst. Solids 307-310, 887 (2002).

8. K. Ngai, L. Bao, A. Yee, C. Soles, Phys. Rev. Lett. 87, 215901 (2001).

9. C. Soles, R. Dimeo, D. Neumann, A. Kisliuk, A. Sokolov, J. Liu, A. Yee, W. Wu, Macromolecules 34, 4082 (2001).

\section{For More Information on this Topic}

W. Wu, M. Cicerone, J. Douglas (Polymers Division, NIST); D. Neumann (NCNR, NIST); A. Sokolov (U. Akron); A. Tsai (Human Genome Services) 



\section{Advanced Manufacturing Methods}

The industrial competitiveness of U.S. manufacturers depends on their ability to reduce the time from product concept to market while decreasing product cost. This is as true for well-established "commodity" industries, such as automotive and aerospace, as it is for rapidly growing or emerging industries, such as biotechnology and nanotechnology. Manufacturing is a critical step in realizing such reductions in product cycle time: for many industries and products, the rapid, low-cost development of manufacturing processes is needed to create new materials with complex product shapes and higher performance at the same or lower cost as the competing, older materials and methods.

To realize such improvements in manufacturing, MSEL is working to develop robust measurement methods, standards, and materials and process data that will help in the design, monitoring, and control of manufacturing processes. An increasingly important step in manufacturing is process modeling. Development of optimized manufacturing processes, such as stamping of complex auto panels or machining of large aircraft components, is time consuming and labor intensive. In many cases, the resulting processes are far from optimal because materials variability, caused, for example, by residual stresses or strain localization during processing, leads to lower yields. Our approach is to develop and combine higher precision, spatially localized measurement methods with realistic, physics-based modeling of manufacturing processes and materials process data to create more useful process modeling tools.

Depending on the stage of the research, our research is often conducted in close collaboration with industrial consortia and standards organizations. This close working relationship developed through these organizations not only ensures the relevance of our research, but also promotes rapid transfer of our research to industry for implementation.

The performance of metallic components in products is strongly dependent on processing conditions that determine microstructural features, such as grain size and shape, texture, the distribution of crystalline phases, macro- and microsegregation, and defect structure and distribution. NIST expertise is applied from a wide range of disciplines, including thermodynamics, electrochemistry, fluid mechanics, diffusion, x-ray, and thermal analysis, to develop measurement methods and understand the influence of processing steps for industries as diverse as automotive, aerospace, microelectronics, and defense. Two major MSEL efforts in Polymer Processing and Forming of Lightweight Metals are centered on unique measurement facilities. Rapidly growing and emerging industries such as biotechnology and nanotechnology are also dependent upon the development of new advanced manufacturing methods that can produce metallic components with the desired characteristics and performance. Related projects with significant manufacturing processes components can be found in the MSEL Programs on Nanocharacterization, Materials for Micro- and Opto-Electronics, and the Interface of Materials with Biology.

\section{Forming of Lightweight Metals}

Automobile manufacturing is a materials intensive industry that involves about $10 \%$ of the U.S. workforce. In spite of the use of the most advanced, cost-effective technologies, this globally competitive industry still has productivity issues related to measurement science and data. Chief among these is the difficulty encountered in die manufacture for sheet metal forming. In the ATP-sponsored workshop (The Road Ahead, June 20-22, 2000), the main obstacle to reducing the time between accepting a new design and actual production of parts was identified as producing working die sets. To benefit from the weight saving of high strength steel and aluminum alloys, a whole new level of formability measurement methods and data is needed, together with a better understanding of the physics behind metal deformation. MSEL is working to fill this need.

\section{Polymers Processing}

Polymers have become ubiquitous in the modern economy because of their ease of processing, high functionality, and low cost. However, these materials can exhibit complex and sometimes catastrophic responses to the forces imposed during manufacturing, thereby limiting processing rates and the ability to predict ultimate properties. The focus of our polymer research is on microscale processing, modeling of processing instabilities, and on-line process monitoring of polymers. Our unique extrusion visualization facility combines in-line microscopy and light scattering for the study of polymer blends, extrusion instabilities, and the action of additives. Current applications focus on understanding and controlling the "sharkskin instability" in polymer extrusion and observation of the dielectric properties of polymer nanocomposites. Fluorescence techniques are developed to measure critical process parameters such as polymer temperature and orientation that were hitherto inaccessible. These measurements are carried out in close collaboration with interested industrial partners.

Contact: Carol A. Handwerker 


\section{Processing Flows \& Instabilities}

The goal of this project is to develop and utilize advanced in situ measurement tools to enable the understanding and control of the complex flows that occur during polymer processing. Systems for study are chosen when the in situ measurements can resolve outstanding scientific and engineering questions. Particular emphasis is on tools to quantify instabilities, interfacial, and multi-component flows.

\section{Kalman B. Migler}

In the extrusion of linear low-density polyethylene (PE), fluoropolymer-processing additives (PPA) are used to eliminate the surface defect known as "sharkskin." Added to the PE at approximately $0.1 \%$ mass fraction, they coat the internal wall of the die during extrusion and provide a slippery surface for the polyethylene, which then slides through undistorted, while reducing energy consumption due to decreased friction.

However, the ability to develop next generation fluoropolymer additives is hampered by a lack of understanding of how they work. In particular, the manner in which they coat the die is not understood; in fact, according to standard fluid mechanics, the fluoropolymer droplets should be repelled by the walls during extrusion.

Working with PPA manufacturers, we have been examining the question of why and where the fluoropolymers coat the die, as well as working to quantitate their behavior once they get there. Past work revealed the details of the sharkskin kinetics and precisely why the fluoropolymers cause the elimination

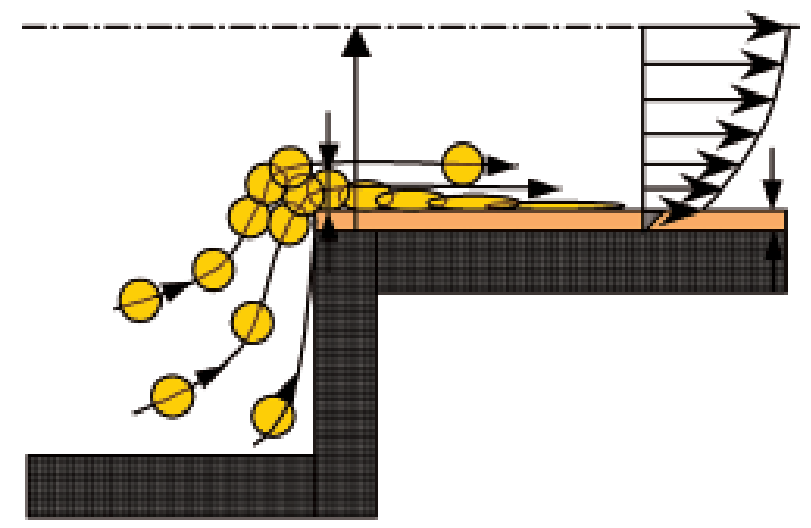

Figure 1: Sketch of the coating process of a fluoropolymer additive onto a solid substrate during the extrusion of polyethylene. of sharkskin. We showed that the PPA coating is not uniform, but, rather, it forms streaks on the die wall.

We recently developed an in situ measurement tool based on frustrated total internal reflection (Frus-TIR) optics. With this technique, we can monitor the coating kinetics in real-time and correlate it with the reductions in sharkskin and energy consumption.

We find that, first, the fluoropolymer coats the upstream region; then, it migrates down the wall to the exit region. When the coating in the exit region is sufficiently established, then sharkskin is eliminated.

We have determined that the coating occurs in the upstream region of the die and that the typical steady state coating thickness ranges from $200 \mathrm{~nm}$ to $500 \mathrm{~nm}$, depending on the extrusion conditions and additive concentrations. However, a coating thickness of less than $60 \mathrm{~nm}$ can also eliminate sharkskin.

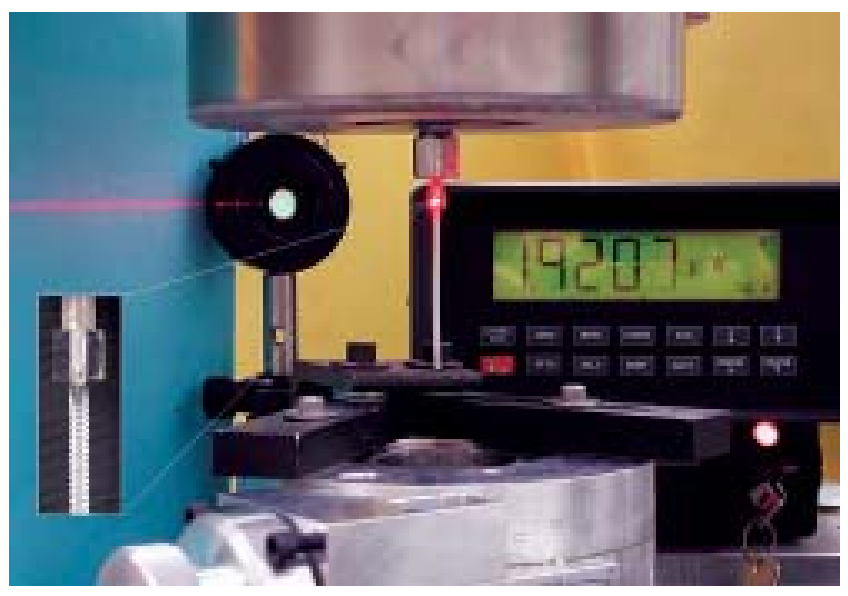

Figure 2: Frustrated Total Internal Reflection optics is installed near the die exit on a capillary rheometer. The amplitude of the reflected light allows a determination of the coating thickness.

We have developed a model of the coating process based on a steady state mass balance equation. This model predicts reasonably well the thickness of the coating. It also gives new insight into the role of the experimental variables on the coating process. For example, we can now make predictions on the effects of throughput, viscosity, entrance angle, and droplet size; these will be explored in future work.

\section{Contributors and Collaborators}

S. Kharchenko, P. McGuiggan (Polymers Division, NIST); M. Meillon, D. Morgan, D. Bigio (University of Maryland); C. Macosko (University of Minnesota); S. Oriani (DuPont Dow Elastomers) 


\section{Combinatorial Methods}

The Combinatorial Methods Program develops novel high-throughput measurement techniques and combinatorial experimental strategies specifically geared towards materials research. These tools enable the industrial and research communities to rapidly acquire and analyze physical and chemical data, thereby accelerating the pace of materials discovery and knowledge generation. By providing measurement infrastructure, standards, and protocols, and by expanding existing capabilities relevant to combinatorial approaches, the Combinatorial Methods Program lowers barriers to the widespread industrial implementation of this new R\&D paradigm.

The Combinatorial Methods Program has adopted a two-pronged strategy for accelerating the development and implementation of combinatorial approaches: an active intramural $R \& D$ program and an ambitious outreach activity. The intramural R\&D program is designed to better tailor combinatorial methods for the materials sciences and extend the state-of-the-art in combinatorial techniques. Measurement tools and techniques are developed to prepare and characterize combinatorial materials libraries, often by utilizing miniaturization, parallel experimentation, and a high degree of automation. A key concern in this effort is the validation of these new approaches with respect to traditional "one at a time" experimental strategies. Accordingly, demonstrations of the applicability of combinatorial methods to materials research problems provide the scientific credibility needed to engender wider acceptance of these techniques in the industrial and academic sectors. The successful adoption of the combinatorial approach also requires a highly developed "workflow" scheme. All aspects of combinatorial research, from sample "library" design and library preparation to high-throughput assay and analysis, must be integrated through an informatics framework, which enables iterative refinement of measurements and experimental aims. Combinatorial Methods Program research projects give illustrations of how combinatorial methodology is implemented effectively in a research setting.

NIST-wide research collaborations, facilitated by the Combinatorial Methods Program, have produced a wide range of new proficiencies in combinatorial techniques, which are detailed in a brochure, "Combinatorial Methods at NIST" (NISTIR 6730), and online at www.nist.gov/combi. Within the Materials Science and Engineering Laboratory (MSEL), novel methods for combinatorial library preparation are designed to encompass variations of diverse physical and chemical properties, such as composition, film thickness, processing temperature, surface energy, chemical functionality, UV-exposure, and topographic patterning of organic and inorganic materials ranging from polymers to nanocomposites to ceramics to metals. New instrumentation and techniques have been developed that enable the high-throughput measurements of adhesion, mechanical properties, failure mechanisms, film thicknesses, and refractive index, among others. The combinatorial effort extends to multiphase, electronic, optoelectronic, and magnetic materials, including biomaterials assays. On-line data analysis tools, process control methodology, and data archival methods are also being developed as part of the Program's informatics effort.

The extensive outreach activity in the Combinatorial Methods Program is designed to facilitate technology transfer with institutions interested in acquiring combinatorial capabilities. The centerpiece of this effort is the NIST Combinatorial Methods Center (NCMC), an industry-university-government consortium organized by MSEL that became operational on January 23, 2002 via a kick-off meeting in San Diego. The recognized importance of NCMC activities is readily apparent, as 15 industrial partners, the Air Force Research Lab, and the University of Southern Mississippi are participating in the NCMC membership program. The membership continues to grow and $80 \%$ of the members from last fiscal year have already renewed for this fiscal year. The NCMC facilitates direct interaction between NIST staff and these industrial partners, and it provides a conduit by which Combinatorial Methods Program research products, best practices and protocols, instrument schematics and specifications, and other combinatorial-related information can be effectively disseminated. This outreach is mediated in large part by a series of semi-annual workshops for NCMC members. Indeed, since its inception, four NCMC workshops have been held at NIST. Further information on the NCMC can be found on the website at www.nist.gov/combi.

Contact: Alamgir Karim 


\section{Polymer Formulations: Fluidic Devices and Scattering Measurements for High Throughput Materials Research}

To meet an increasing demand for combinatorial approaches to polymer formulations science, we are developing fluid handling and measurement methods to extend combinatorial and high-throughput (C\&HT) materials science to complex fluids. Two examples are highlighted here. Solvent resistant fluid handling devices with channels of tunable dimensions are fabricated within a few hours using a novel rapid prototyping technique, and a complimentary approach to measure formulation microstructure on arrays of mixtures is developed using $x$-ray scattering.

\section{Kathryn Beers}

A gauged by the response of NIST Combinatorial Methods Center (NCMC) members, one of the greatest opportunities for contribution of combinatorial methods is in the area of complex fluid formulation. The ability to manipulate and measure multi-component mixtures with C\&HT methods is attracting considerable industrial and academic interest.

We have developed a rapid prototyping technique able to produce solvent-resistant fluidic devices. ${ }^{[1]}$ It is based on the contact lithography of a UV curable optical adhesive and requires only a mask (a laser printed transparency or image setter) and a conventional $365 \mathrm{~nm}$ UV source. Fluid handling devices with channels of tunable dimensions (of the order of $100 \mathrm{~s}$ of $\mu \mathrm{m}$ ) are fabricated within a few hours.

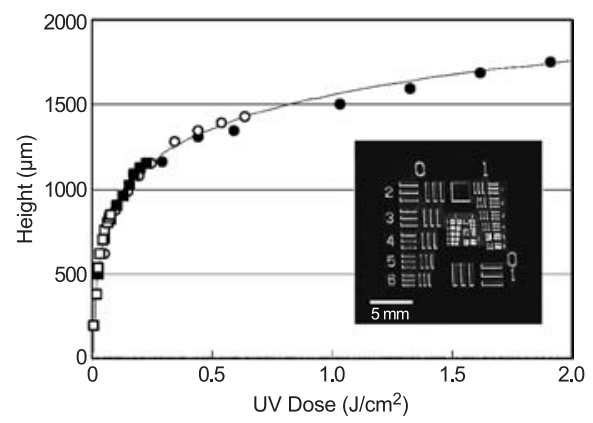

Figure 1: Height of patterned structures as a function of administered UV dose; symbols are experimental data and the solid line is a logarithmic fit. ${ }^{[2]}$ The inset shows a patterned resolution target (USAF 1951) reproducing linewidths (in-plane dimensions) of $70 \mu \mathrm{m}$ and above.

An example of such a device is described in the Nanoscale Manufacturing project in this report and is the basis of a Focused Project established with industrial collaborators through the NCMC to develop an HT method to measure interfacial tension. Additional current applications include devices for in situ polymerization, combinatorial mixers, stamps for microcontact printing, ${ }^{[3]}$ and substrates for discrete composition libraries. With these library substrates, a home-built liquid dispenser, and automated light scattering, we are investigating the phase behavior of multi-component polymer formulations.

Through the development of this fabrication technique, a complimentary approach to measure scattering from a discrete composition library was also demonstrated. The substrate, a polydimethylsiloxane (PDMS) array, was capped with polyimide windows. The library was investigated by Small Angle X-Ray Scattering (SAXS) at the National Synchrotron Light Source at Brookhaven National Laboratory. This model library contained compositions of aqueous ethyleneoxide (EO) butyleneoxide $(\mathrm{BO})$ block copolymer, $\mathrm{EO}_{6} \mathrm{BO}_{11}$, which is known to produce vesicle structures at low concentration. ${ }^{[4]}$ The vesicles are micrometers in size, which makes this system an excellent model to be used in developing additional C\&HT techniques using a variety of in-house scattering systems.

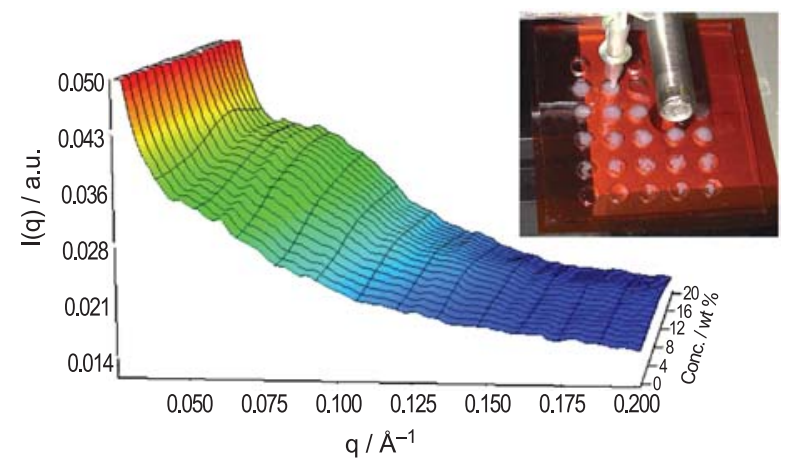

Figure 2: $I(q)$ vs. $q$ vs. concentration for the SAXS data from $E O_{6} \mathrm{BO}_{11}$ in water. Evolution of lamellar structure within the vesicle and the absence of crystallinity are both evident. The inset shows the PDMS/polyimide array.

Future work includes developing a suite of processing capabilities using the prototyping technique and interfacing the formulations libraries prepared with new measurement methods being developed in parallel.

\section{References}

1. C. Harrison, J.T. Cabral, C.M. Stafford, A. Karim, and E.J. Amis, (submitted).

2. Cabral, et al., (in preparation).

3. Sehgal, et. al., (in preparation).

4. J.K. Harris, et al., Langmuir, 2002, 18, 5337-5342.

\section{Contributors and Collaborators}

J.T. Cabral, A.I. Norman, A. Karim, E.J. Amis (Polymers Division, NIST) 


\section{Combinatorial Epoxy Curing Library: Correlating Network Formation, Chemical Conversion, and Adhesion}

The performance of epoxy-based advanced composites, coatings, and structural adhesives depends strongly on the resin curing process. Thus, combinatorial and high-throughput methods are ideally suited to study the curing process. We are working to correlate epoxy film curing properties as a function of two processing variables using three independent techniques: FTIR reflectance microscopy, confocal microscopy, and axisymmetric adhesion tests.

\section{Aaron M. Forster, Naomi Eidelman, and Dharmaraj Raghavan}

$\mathrm{U}$ sing gradient and high-throughput techniques, we analyzed epoxy curing as a function of both temperature and time. Diglycidyl ether bisphenol A epoxy resin was mixed with fluorescent dye (4 dimethylamino-4'-nitrostilbene) and curing agent (2,4,6-tri(dimethyl amino methyl) phenol) and deposited via flow coating on a glass substrate. The epoxy film sample was cured on a temperature gradient stage, (50 to 100 ) ${ }^{\circ} \mathrm{C}$, for $(15,30,45,60$, and 180 ) min, and $39 \mathrm{~h}$. The epoxy film was mapped ex-situ by Fourier Transform Infrared (FTIR)-reflectance microscopy and confocal microscopy, in conjunction with adhesion tests (contact with polydimethylsiloxane (PDMS) hemispherical lens) to provide chemical, physical, and interfacial information on the epoxy film during the curing process.

The chemical conversion of epoxy groups is measured with FTIR. As shown in Figure 1, color maps of epoxy conversion quantify chemical changes as a function of the two variables.

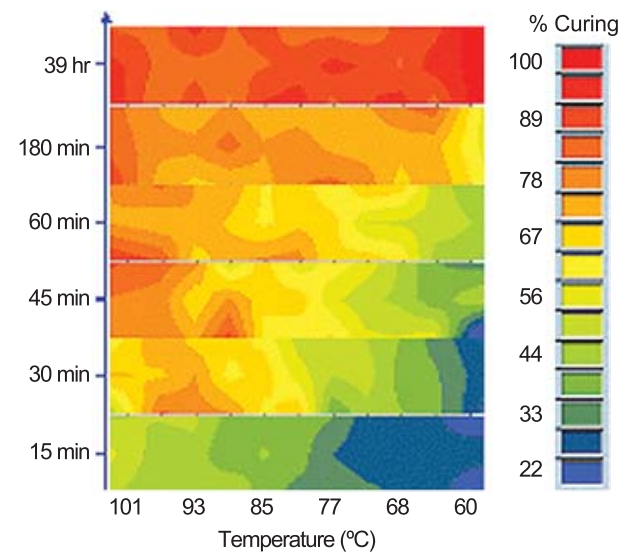

Figure 1: FTIR maps of an epoxy film at different curing temperatures and times. The colors indicate percent curing.
Confocal microscopy measurements of fluorescence intensity (emission maximum) characterize epoxy network formation. The cured epoxy film network restricts fluorophore motion, which leads to a higher fluorescence intensity relative to the uncured epoxy film (see Figure 2).

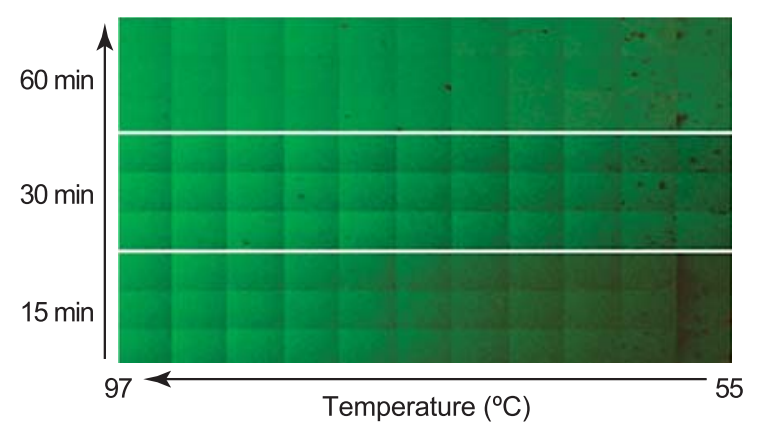

Figure 2: The epoxy resin film exhibits increases in the fluorescence intensity at higher curing temperatures or longer curing times.

Adhesion measurements probe the chemical and mechanical properties near the interface. These properties change as the epoxy film cures. The work of adhesion to detach a PDMS lens from the epoxy surface is shown in Figure 3.

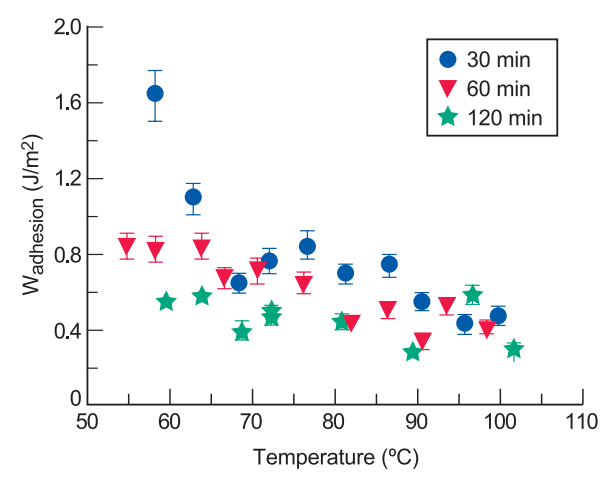

Figure 3: The epoxy resin film is tackier at low temperatures and short curing times, which leads to a larger work of adhesion.

Future work will focus on: the quantitative comparison between these independent techniques, the epoxy network structure at low- and high-curing temperatures, and the epoxy-PDMS interface to better correlate adhesion measurements to epoxy properties.

\section{Contributors and Collaborators}

A. Karim, E.J. Amis (Polymers Division, NIST); N. Eidelman (American Dental Association Foundation, PRC, NIST); D. Ragahavan (Howard University) 


\section{NIST Combinatorial Methods Center (NCMC) A Partner in Accelerating Materials Research}

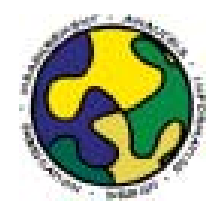

\begin{abstract}
Combinatorial and high-throughput (C\&HT) techniques hold great potential for materials research, but significant barriers prevent the widespread adoption of these revolutionary methods. Through responsive outreach and creative, cost-effective measurement solutions, the NIST Combinatorial Methods Center strives to ease the acquisition of C\&HT techniques by the materials research community.
\end{abstract}

\section{Cher H. Davis and Michael J. Fasolka}

“As Honeywell Specialty Materials initiates our combinatorial effort, NIST offers an invaluable resource [as to] what can be done, and how to go about it. The NCMC clearly demonstrates that a combinatorial effort does not imply multi-million dollar expenditures..."

S. Curran, A. Signorelli, Honeywell International

$\mathrm{T}_{-}^{\mathrm{h}-\mathrm{s}}$ he NIST Combinatorial Methods Center (NCMC) - now in its second year - has attracted

16 member partners from industry and government. Since its inception, the NCMC has tailored its research and outreach towards current and emerging industrial needs in C\&HT methods. As part of its FY2003 outreach, the NCMC organized three workshops. The first, NCMC-2, was held October 7-8, 2002 at NIST. NCMC-2 related NCMC-developed C\&HT measurement methods for mechanical properties and adhesion (including SIEBIMM, see associated Highlight). In addition, the NCMC-2 program included a plenary symposium, facilities tours, and a panel discussion that revealed industrial needs for a Focused Project in HT Interfacial Tension Measurements (now launched, see http://www.nist.gov/combi).

"The people of the NCMC are developing elegant, innovative screens. I think we're getting more than our money's worth."

- M.S. Vratsanos, Air Products and Chemicals

Two additional workshops were aimed at initiating NCMC actions in C\&HT Informatics. Needs in the field were gleaned at the NCMC Combinatorial Informatics industrial symposium held in conjunction with a Knowledge Foundation Meeting on February 19, 2003 in San Jose, CA. Analysis of industry concerns indicated two areas that presented a NIST role. First, industry requires examples of how to integrate automated fabrication instruments, characterization devices, and analysis routines into a database-centered informatics system suitable for C\&HT research. Second, interoperability barriers hamper informatics system development. In particular, the lack of a common data interchange format greatly complicates the integration of devices from disparate vendor sources into a coherent C\&HT system.
In response to these needs, the NCMC is building a model C\&HT informatics system in its laboratory facilities. Constructed with open-source code, the NCMC system provides transparent examples of informatics infrastructure. In addition, the NCMC endeavors to help create data interchange format standards for the C\&HT materials research community. Both efforts were highlighted at NCMC-3: Combinatorial informatics, held at NIST on May 22-23, 2003. This workshop outlined issues in C\&HT informatics and several NIST XML-based interchange formats relevant to materials research. $N C M C-3$ culminated with the formation of the Combinatorial Materials Research Data Standards (CMRDS) Working Group - a first success toward establishing data standards for the C\&HT materials community. Ten NCMC-3 attendees volunteered to serve on CMRDS committees, which are aimed at identifying opportunities (e.g., XML) and at defining components for a data interchange format. For details, see the CMRDS website at http://polymers.msel.nist.gov/combi/cmrds.html.

"[NIST is] doing a great job! The NCMC is an opportunity for us to benefit from the excellent work being conducted by that group. ... In the long term, this technology could potentially change the way $R \& D$ is conducted across the world." Y. Dar, ICI/National Starch \& Chemicals

FY2004 promises sustained effort and new directions from the NCMC. NCMC-4: Polymer Formulations, to be held October 6-7 2003, will feature C\&HT measurement strategies for complex fluids and sessions dedicated to the Interfacial Tension Focused Project and the CMRDS Working Group. Moreover, new Focused Projects in Formulations and Adhesion will be launched. In addition, a new NCMC effort in nanotechnology will create reference substrates designed to test and calibrate advanced scanning probe microscopy measurements. For more information on these efforts and other NCMC programs, see http://www.nist.gov/combi.

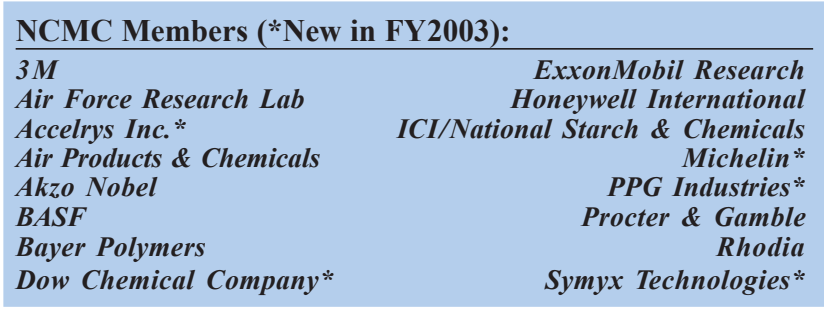

\section{Contributors and Collaborators}

A. Karim, W. Zhang, K.L. Beers, C. Stafford, A. Forster, H.J. Walls, J.T. Cabral, A.I. Norman, D.H.A. Chiche, E.J. Amis (Polymers Division, NIST) 


\section{Interface of Materials with Biology}

New materials and devices are radically changing the medical treatment of injury and disease, yet because of the rapid pace of this segment of the materials industry, an adequate measurement infrastructure does not yet exist. The program on the Interface of Materials with Biology develops measurement methods, standards, and fundamental scientific understanding at the interface between materials science and biological science. Within the health care industry, we focus on dental and medical sectors that apply synthetic materials for replacement, restoration, and regeneration of damaged or diseased tissue. Three primary foci exist within this program: biocompatibility, materials properties, and materials science techniques applied to biological systems.

Whether the medical issue involves implanting a hip or knee joint prosthesis, a synthetic bone graft, or a tissue engineering scaffold into the human body, one of the primary issues is biocompatibility. By using our expertise in materials science, we are working to develop suitable Reference Materials (RM) for investigating biocompatibility and implant suitability. Research has focused on measuring the cellular response to powders and bulk materials to identify suitable candidates. We are collaborating with the American Dental Association Foundation (ADAF) to develop metrology methods to characterize the biocompatibility of synthetic bone grafts. Quantitative methods being developed include assays for adhesion, viability, proliferation, and differentiation of bone cells, as well as optical coherence tomography and confocal microscopy for measuring tissue ingrowth. We are developing biochemical assays to quantify inflammatory responses to synthetic materials. Finally, current research is working to bridge the gap between knowledge generation by cell biologists and product development in industry. In collaboration with the Chemical Science and Technology Laboratory, we are developing measurement methodologies and reference materials to use in assessing interactions in complex systems of living cells with synthetic materials.

The expected outcomes of this work are reference substrates that induce specific cellular responses and engineered DNA vectors to act as fluorescent reporters of cellular responses.

In addition to the issue of biocompatibility, it is critical that the materials can withstand the mechanical and environmental stresses placed on them. For metallic implants, one concern is the corrosion pitting resistance of the implant materials and the associated potential for stress corrosion cracking (SCC). To address this issue, metal standards are being subjected to a simulated biological environment which will then be used to develop tests to assess the susceptibility for SCC.

Mechanical properties issues also arise when considering synthetic bone grafts and tissue engineering scaffolds. In addition to traditional bulk mechanical property measurements, combinatorial approaches are used to identify compositions and surface features that affect properties such as biocompatibility and mechanical durability. Finally, because the dental industry is primarily composed of small manufacturers with limited R\&D capability, collaborations with the ADAF, located in MSEL, are filling the gap by developing improved materials and techniques, patenting and licensing these inventions, and, most importantly, providing a technical foundation. Research focuses on improved understanding of the synergistic interaction of the phases of polymer-based composites and the mechanisms of adhesion to dentin and enamel. This approach will ultimately lead to materials with improved durability, toughness, and adhesion to contiguous tooth structure.

In this era of interdisciplinary approach to research, we provide an added dimension. By taking a physical/mechanical approach to how cells function, respond, and remodel in interaction with synthetic materials, we can provide skill sets typically absent in the biomedical community. Our concentration on mechanical property metrology extends to biological systems, spanning a considerable size range from individual neurons and muscle cells to complete pulmonary arteries. This necessitates the development of unique mechanical testing platforms and application of a materials science approach to understanding integrated properties.

Fundamental to much of the work in this program is the recognition that surfaces and interfaces play a critical role in biological systems and, in particular, in the interactions of synthetic or designed materials with biological systems and function. By applying the expertise in the NIST Materials Science and Engineering Laboratory to characterization of surfaces and interactions at interfaces in biomaterials, we will accelerate the introduction of improved materials and help provide the means to assure quality control that is critical to this industry.

Contact: Newell R. Washburn 


\section{Bioactive Polymeric Composites for Mineralized Tissue Regeneration}

\begin{abstract}
There is a growing interest in the biomaterials community about polymer-based bioactive materials that can promote the preservation/ remineralization of hard dental tissues, i.e., enamel, dentin, and bone. As part of a joint research effort with the American Dental Association Foundation-Paffenbarger Research Center (supported by the National Institute of Dental and Craniofacial Research), NIST has been investigating various types of polymeric calcium phosphate composites. As a result, NIST is providing the dental industry and profession with a comprehensive knowledge base regarding these potentially bioactive materials that will help elucidate their in-vitro and in-vivo performance.
\end{abstract}

\section{Joseph M. Antonucci}

$\mathrm{C}$ alcium phosphates are of special interest to oral biology, dentistry, and medicine since they occur in normal skeletal tissues and in the pathological calcification of nonmineralized tissues and synthetic materials. Crystalline hydroxyapatite (HAP) is the structural prototype of the major mineral component of teeth and bones. Amorphous calcium phosphate (ACP), a postulated precursor to biological HAP, shows high solubility/degradability in aqueous media, readily liberating calcium and phosphate ions, and transforming to crystalline apatitic calcium phosphate. These properties suggested its use as a bioactive filler in polymeric dental composites.

In recent studies, we have applied solid-state ${ }^{1} \mathrm{H}$ NMR methods to better characterize ACP. We can distinguish between rigid waters of hydration in the lattice, plus both strongly and weakly bound water. Hydroxy protons in apatitic structures can also be identified. In ACP, about $2 / 3$ of the protons are in rigid waters of hydration; however, the fractions of strongly and weakly bound water vary with pyrophosphate, an ACP stabilizer used to slow the conversion to apatitic structures.

In other studies, we have examined the effect of acidic comonomers on the degree of vinyl conversion (FTIR) and mechanical strength (biaxial flexural strength) of ACP composites formulated with equal amounts of 2,2-bis[p-(2'-hydroxy-3'-methacryloxypropoxy) phenyl] (Bis-GMA) and triethylene glycol dimethacrylate (TEGDMA). As expected, the conversion values for the composites are somewhat reduced compared to those of the unfilled copolymers. Significantly, with these systems, the differentials between the vinyl conversion of the unfilled copolymers and their composites were smaller than that observed for other types of ternary Bis-GMA/TEGDMA systems. With one acidic termonomer system, where the acid monomer was relatively hydrophobic, a significant enhancement of biaxial flexure strength, even after prolonged aqueous storage, was observed, indicating the effect of chemical structure of the polymer matrix on interfacial properties.

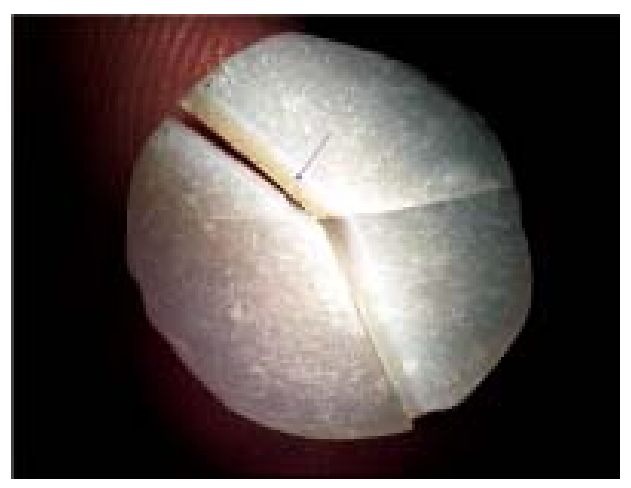

Figure 1: ACP composite disk (16 $\mathrm{mm} D \times 1 \mathrm{~mm} \mathrm{H})$ after biaxial flexure loading. Note the polyethylene fibers bridging the crack near the center of the fractured disk.

A problem with dental composites of all types is their lack of sufficient toughness to resist cracking under masticatory stress. This can be especially critical in the case of ACP composites that are significantly mechanically weaker than glass-filled composites. In a preliminary study, we found that a urethane dimethacrylate commonly used in formulating dental composites can be blended (3/1 ratio by mass) with a more flexible PEG400 diol urethane dimethacrylate to yield tough ACP composites (as measured by stress-strain curves). In addition, it was found that the addition of small amounts $(0.1 \%$ mass fraction) of chopped polyethylene fibers resulted in composites that did not fail catastrophically under biaxial stress while maintaining their biaxial flexure strength (see Figure 1). It is expected that this developing knowledge base will aid future research and prove of value to manufacturers in their design of more efficacious therapeutic dental and biomaterials.

\section{Contributors and Collaborators}

W.G. McDonough, D. Vanderhart, D. Liu (Polymers Division, NIST); D. Skrtic, J.B. Quinn, G.E. Schumacher (American Dental Association Foundation-Paffenbarger Research Center); W.F. Regnault (FDA, CDRH) 


\section{High-Throughput Biocompatibility Screening of Polymer Blends}

\begin{abstract}
The Polymers Division is developing high-throughput methods for the rapid screening of biomaterials. We have prepared single samples with gradients of polymer composition in the form of a film onto which cells can be cultured. Measurement of cellular response on these gradients allows rapid characterization of cellular response as a function of material properties.
\end{abstract}

\section{Carl G. Simon, Jr. and Newell R. Washburn}

$\mathrm{P}_{\mathrm{p}}^{\mathrm{o}}$ olymers are frequently blended to optimize material properties such as strength, flexibility, morphology and crystallinity. This includes polymers used for biomedical applications. For instance, there are commercially available degradable sutures made of Polyglactin 910, which is a blend of poly(L-lactide) (PLLA) and poly(lactide-co-glycolide). In order to speed the development of new biomaterials, we are developing high-throughput methods for characterizing the biocompatibility of polymer blends.

Preparation of individual polymer specimens of discreet compositions that systematically span a significant range of properties can be tedious and time-consuming. To address this, we have used a high-throughput technique that involves the creation of a gradient of polymer composition in the form of a film to examine cell response to blends of two polymers. Cell responses such as adhesion, morphology, proliferation, and differentiation can be evaluated after culturing cells on the polymer composition gradients. Compositions that support cell function can be rapidly identified to provide an optimal blend composition for use in tissue engineered medical products.

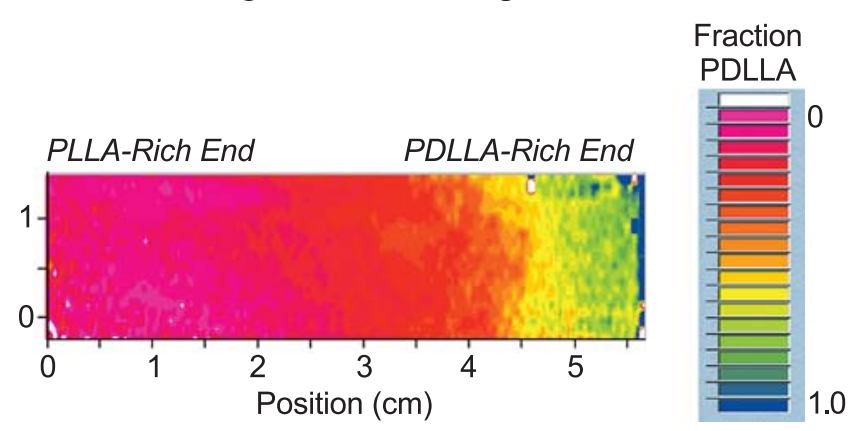

Figure 1: FTIR map of a PLLA-PDLLA composition gradient.

We have made composition gradients of two biodegradable polymers, PLLA and poly(D,L-lactide) (PDLLA). The pure polymer solutions are separately and simultaneously infused into, and withdrawn from, a mixing vial such that the composition of the resulting mixture changes over time. There, we use a syringe to sample the solution during mixing, which results in a composition gradient along the barrel of the syringe. The gradient solution is then quickly deposited in a line on a flat substrate and spread into a film using a knife.

The gradients are characterized with microspectroscopic fourier transform infrared (FTIR) spectroscopy to map composition of the entire gradient library (Figure 1) and atomic force microscopy (AFM) to map surface topology. FTIR reveals that the technique is feasible and makes consistent gradients. AFM shows that the surface roughness varies with composition. The PLLA-rich end of the gradient (RMS $\approx 10 \mathrm{~nm}$ ) is about 10 times rougher than the PDLLA-rich end $(\mathrm{RMS} \approx 1 \mathrm{~nm})$.

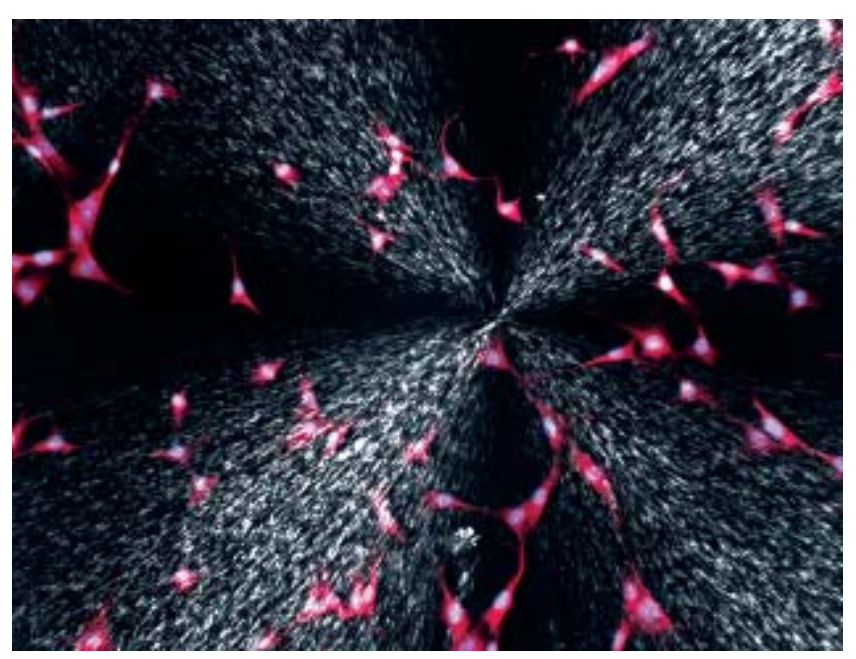

Figure 2: Osteoblasts growing on a PLLA spherulite.

Cellular response is characterized on the library as a function of local material parameters. We are using automated fluorescence microscopy to correlate changes in cell shape, cytoskeleton development, focal adhesions, and proliferation, and correlating these with substrate topography. Preliminary studies with osteoblast-like cells (MC3T3-E1) suggest that they proliferate faster on PDLLA. This technique should accelerate materials research for tissue engineering by providing a rapid technique for investigators to screen polymer blends for their ability to support cell function.

\section{Contributors and Collaborators}

A. Karim, S. Kennedy, A. Sehgal, C. Khatri, F. Wang, C. Stafford and M. Weir (Polymers Division, NIST); N. Eidelman (American Dental Association) 


\section{Measurement of Cellular Response to Three-Dimensional Scaffolds for Tissue Engineering}

In the Polymers Division, we are developing measurement methods for determining structure-property relationships for tissue engineering scaffolds. The performance of this new class of medical devices is a complex function of scaffold composition, geometry, and embedded growth factors. We have established bioreactor culturing methods capable of promoting tissue development in these three-dimensional scaffolds and are characterizing the influence of systematic variations in scaffold geometry on bone development.

\section{Newell Washburn and James Cooper}

$\mathrm{T}$ The tissue engineering industry is faced with many measurement issues when trying to characterize porous, three-dimensional (3-D) scaffolds. Porous, 3 -D scaffolds are needed to regenerate tissue growth within the implant for stronger fixation. Unlike flat surfaces used to grow cells in culture, normal cells and tissue in the body are in a three-dimensional environment that determines cellular response.

Placing the cells in a 3-D environment sends signals to the cells that allow the cells to differentiate to the correct phenotype. We are developing measurement methods and protocols to monitor cell migration and matrix formation within scaffolds as a function of scaffold geometry.

The ability to change the porosity and poreinterconnectivity of the 3-D scaffold will give more insight into the environment needed for proper cellular response and varies the measurement environments for identification of cells and its components. In Figure 1, representative images of 3-D polymeric scaffolds prepared by co-extrusion show changing pore interconnectivity with pore diameters $>100 \mu \mathrm{m}$.

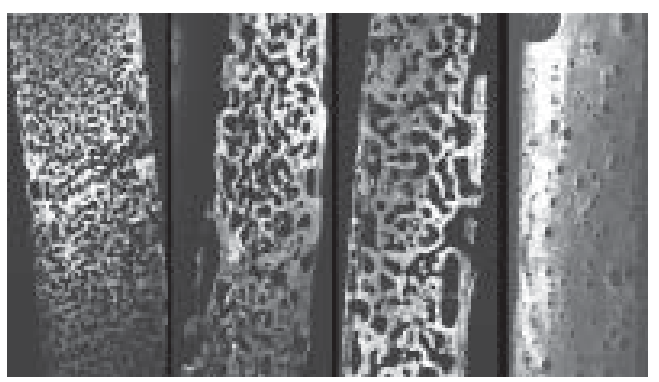

Figure 1: Cross-sections of 3-D polymer scaffolds showing the capability to control pore-interconnectivity in order to measure cellular response to changing porosity and pore diameters (small to large).

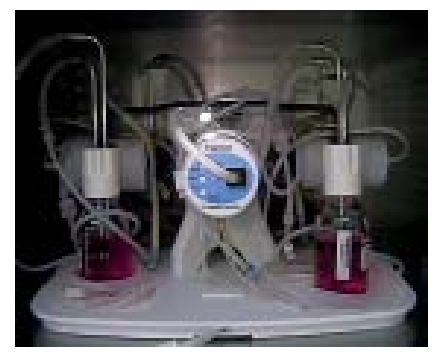

Figure 2: Perfusion bioreactor used to expose cells to dynamic flow conditions.

The rate at which the cells migrate into a 3-D scaffold and initiate matrix formation is crucial to the development of tissue engineered scaffolds. Once implanted, the cells within the 3-D scaffolds must send signals to outer migrating cells to reduce the inflammatory response and start the remodeling phase of healing. In order to measure cellular response to dynamic environments, we use a perfusion bioreactor (Figure 2). The perfusion bioreactor allows simultaneous nutrient supply and removal of cellular waste. The bioreactors also stimulate the cells with shear forces due to the flow of media. In addition, other studies have shown an increase in cell proliferation and matrix production by using bioreactor systems.

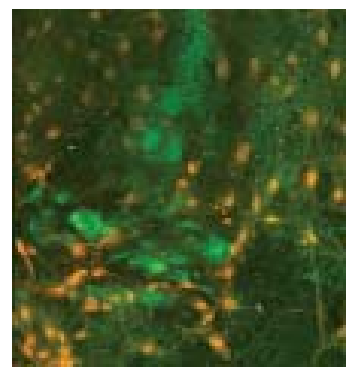

Figure 3: Fluorescent image of cell migration (cell nucleiorange) on the surface of 3-D PCL scaffolds (green).

Once seeded on the structure, cell viability, proliferation, and infiltration into the porous scaffold network is important to the development of new tissue. Quantitative 3-D imaging techniques are being developed to measure organization of cells and extra-cellular matrix components to determine migration and differentiation. The fluorescence image in Figure 3 shows MC3T3-E1 cells (osteoblastic cell line) on the surface of a 3-D polymeric scaffold.

\section{Contributors and Collaborators}

J. Dunkers, M. Cicerone, C. Simon (Polymers Division, NIST) 


\section{Materials for Micro- and Opto-Electronics}

U.S. microelectronics and related industries are in fierce international competition to design and produce smaller, lighter, faster, more functional, and more reliable electronics products more quickly and economically than ever before. At the same time, there has been a revolution in recent years in new materials used in all aspects of microelectronics fabrication.

Since 1994, the NIST Materials Science and Engineering Laboratory (MSEL) has worked closely with the U.S. semiconductor, component, packaging, and assembly industries. These efforts led to the development of an interdivisional MSEL program committed to addressing industry's most pressing materials measurement and standards issues central to the development and utilization of advanced materials and material processes. The vision that accompanies this program - to be the key resource within the Federal Government for materials metrology development for commercial microelectronics manufacturing - may be realized through the following objectives:

- Develop and deliver standard measurements and data;

- Develop and apply in situ measurements on materials and material assemblies having micrometer- and submicrometer-scale dimensions;

- Quantify and document the divergence of material properties from their bulk values as dimensions are reduced and interfaces contribute strongly to properties;

- Develop models of small, complex structures to substitute for or provide guidance for experimental measurement techniques; and

- Develop fundamental understanding of materials needed in future micro- and opto-electronics.

With these objectives in mind, the program presently consists of projects led by the Metallurgy, Polymers, Materials Reliability, and Ceramics Divisions that examine and inform industry on key materials-related issues. These projects are conducted in concert with partners from industrial consortia, individual companies, academia, and other government agencies. The program is strongly coupled with other microelectronics programs within government and industry, including the National Semiconductor Metrology Program (NSMP) at NIST. Materials metrology needs are also identified through industry groups and roadmaps, including the International Technology Roadmap for Semiconductors (ITRS), the IPC Lead-free Solder Roadmap, the National
Electronics Manufacturing Initiative (NEMI)

Roadmap, the Optoelectronics Industry Development

Association (OIDA) roadmaps, and the National [Magnetic Data] Storage Industry Consortium (NSIC).

Although there is increasing integration within various branches of microelectronics and optoelectronics, the field can be considered in three main areas. The first, microelectronics, includes needs ranging from integrated circuit fabrication to component packaging to final assembly. MSEL programs address materials metrology needs in each of these areas, including lithographic polymers and electrodeposition of interconnects, electrical, mechanical, and physical property measurement of dielectrics (interlevel, packaging, and wireless applications), and packaging and assembly processes (lead-free solders, solder interconnect design, thermal stress analysis, and co-fired ceramics).

The second major area is optoelectronics, which includes work which often crosses over into electronic and wireless applications. Projects currently address residual stress measurement in optoelectronic films, optical and structural characterization of wide bandgap semiconductors, and standards development for III-V compound semiconductors. Cross-laboratory collaborations with EEEL figure prominently in this work.

The third area is magnetic data storage, where the market potential is vast and growing and the technical challenges extreme. NSIC plans to demonstrate a recording density of 1 terabit per square inch - 40 times today's level - by 2006. To reach these goals, new materials are needed that have smaller grain structures, can be produced as thin films, and can be deposited uniformly and economically. New lubricants are needed to prevent wear as spacing between the disk and head becomes smaller than the mean free path of air molecules. Some measurements require calibration of magnetometers using certified magnetic standards in several different shapes and magnetic strengths, and with a wide range in magnetic character. MSEL is working with the magnetic recording industry to develop measurement tools, modeling software, and standards to help achieve these goals, with MSEL, the Electronics and Electrical Engineering Laboratory, the Physics Laboratory, the Information Technology Laboratory, and the Manufacturing Engineering Laboratory working as partners in this effort.

Contact: Frank W. Gayle 


\section{Characterization of Porous Low-k Dielectric Constant Thin Films}

\begin{abstract}
NIST is working to provide the semiconductor industry with unique on-wafer measurements of the physical and structural properties of porous thin films important to their use as low-k dielectric materials. The methodology utilizes several complementary experimental techniques to measure the average pore and matrix morphology. Pore size distributions are calculated by $x$-ray reflectivity and neutron scattering porosimetry. Methods have been developed to measure closed pore content and matrix morphology in films having complex structures with any morphological type.
\end{abstract}

\section{Barry J. Bauer}

$\mathrm{T}$ he next generation of integrated circuits will require feature sizes that demand new low-k interlayer dielectric materials. To address problems with power consumption, signal propagation delays, and crosstalk between interconnects, dielectric constants need to significantly decrease. The primary approach to low-k dielectric materials is the introduction of nanometer scale pores into a solid film to lower its effective dielectric constant. While these modifications change the dielectric constant favorably, other important parameters such as physical strength and barrier properties will also change, often in an unfavorable way. Characterization of the pore structure is crucial to the development of new dielectric materials, and new measurement methods are necessary.

NIST provides low-k characterization using a combination of small angle neutron scattering (SANS), high-resolution x-ray reflectivity (HRXR), and ion scattering techniques. These measurements are performed directly on films supported on silicon substrates so that processing effects can be investigated. New methods are required, however, to provide more detailed information necessary to complete the product development.

This year, we have continued our efforts on characterization of current industrially relevant materials primarily through International SEMATECH, a consortium of microelectronics companies, but also through other industrial partners. The technological roadmap has progressed to a decision point at which materials will be chosen for the next step: the integration of low-k materials into complex circuits. NIST has provided a considerable body of data to make this choice possible.

To advance the measurement capabilities, we have perfected new techniques for more thorough and accurate film characterization. Work has been completed on a small angle neutron scattering (SANS) technique and instrumentation for measurement of films that have been infused by mixtures of normal solvent and the same solvent in which the hydrogens have been replaced by deuterium (see Figure 1). The match point measures where the neutron contrast matches that of the wall material. By filling the open pores with contrast match fluids, SANS reveals the closed pore content and size. The relative open and closed pore content is becoming increasingly important to low-k applications. The same instrumentation has been used to determine the uniformity of the wall material with respect to remnants of porogen that remain after incomplete thermal treatment.

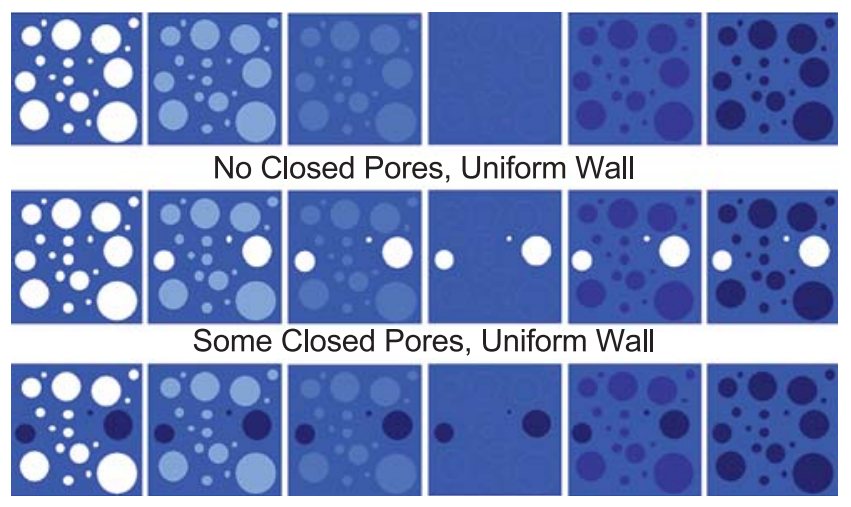

No Closed Pores, 2 Phase Wall

Figure 1: Schematic illustrating different sample characteristics that can be distinguished using SANS contrast match techniques.

This year, several projects have been completed in response to industry needs. SANS has been used to measure the size and volume fraction limits of damaging "killer" pores in collaboration with Dow Chemical. $\mathrm{X}$-ray reflectivity has been used to measure the effects of plasma treatment on film density profiles in collaboration with Axcelis. The pore formation process was quantified to connect pore size distributions of the formed pores with pore size distributions of the porogen used to generate the pores in collaboration with Dow Chemical. A new method of calculating pore size distributions from SANS data has been demonstrated.

\section{Contributors and Collaborators}

H. Lee, R. Hedden, C. Soles, M. Silverstein, D. Liu, W. Wu (Polymers Division, NIST); C. Glinka (Center for Neutron Research, NIST); J. Iacoponi, Y. Liu (International SEMATECH); B. Landes (Dow Chemical); C. Waldfried, O. Escorcia (Axcelis); B. Hwang, W. Gray (Dow Corning) 


\section{Polymer Photoresists for Next-Generation Nanolithography}

Photolithography, the process used to fabricate integrated circuits, is the key enabler and driver for the microelectronics industry. As lithographic feature sizes decrease to the sub $100 \mathrm{~nm}$ length scale, significant challenges arise because both the image resolution and the thickness of the imaging layer approach the macromolecular dimensions characteristic of the polymers used in the photoresist film. Unique high-spatial resolution measurements are developed to reveal limits on materials and processes that challenge the development of photoresists for next generation sub-100 nm lithography.

\section{Eric K. Lin}

$\mathrm{P}$ hotolithography is the driving technology used by the microelectronics industry to fabricate integrated circuits with ever decreasing sizes. In addition, this fabrication technology is rapidly being adopted in emerging areas in optoelectronics and biotechnology requiring the rapid creation of nanoscale structures. In this process, a designed pattern is transferred to the silicon substrate by altering the solubility of areas of a polymeric photoresist thin film through an acid catalyzed deprotection reaction after exposure to radiation through a mask. To fabricate smaller features, next generation photolithography will be processed with wavelengths of light requiring photoresist films less than $100 \mathrm{~nm}$ thick and dimensional control to within $2 \mathrm{~nm}$. Material, interface, and transport issues arise when fabricating structures over these length scales. To date, the materials and processes needed to fabricate sub-100 $\mathrm{nm}$ features have not been selected because of increasingly challenging technical issues.

To advance this key fabrication technology, we work closely with industrial collaborators to develop and apply high-spatial resolution and chemically specific measurements to understand changes in material properties, interfacial behavior, and process kinetics at nanometer scales that can significantly affect the patterning process.

This year, we have applied and enhanced unique measurement methods to provide structural measurement of fabricated nanoscale structures and new insight and detail into the complex physico-chemical processes used in advanced chemically amplified photoresists. These methods include $x$-ray and neutron reflectivity (XR, NR), small angle neutron and x-ray scattering (SANS, SAXS), incoherent neutron scattering (INS), near-edge x-ray absorption fine structure spectroscopy (NEXAFS), combinatorial methods, nuclear magnetic resonance
(NMR), quartz crystal microbalance (QCM), Brillouin light scattering (BLS), fluorescence correlation spectroscopy (FCS), and atomic force microscopy (AFM).

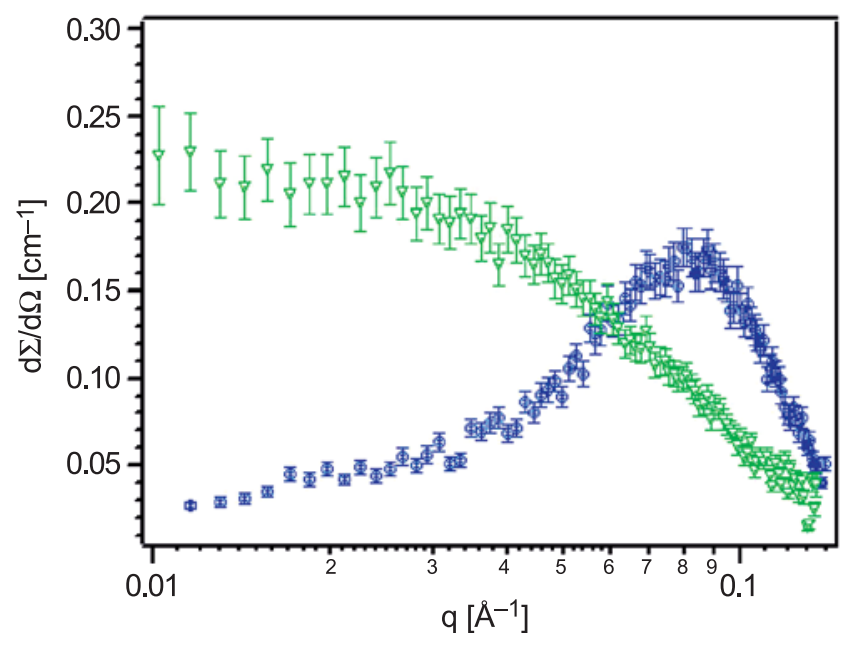

Figure 1: SANS data from a model photoresist polymer in organic solvent (green) and aqueous base developer (blue). The peak in the blue curve shows electrostatic effects on the chain conformation not present in the organic solvent.

Accomplishments for this past year include: advancements in critical dimension measurement with SAXS (see highlight); identification of polyelectrolyte effects in photoresist dissolution (see Figure 1) and their impact on the resolution of the structure; direct measurement of surface segregation of a fluoropolymer blend component to the surface of the resist film impacting its dissolution behavior; identification of key anti-reflection coating (ARC) components responsible for profile control problems at the ARC-resist interface; correlation of polymer local dynamics with transport of water and photoacid diffusion showing the effect of confinement on resist processing parameters; and the first measurement of the shape and form of the 3-D deprotection volume from the reaction-diffusion of dispersed photoacids, a key variable in model predictions of the roughness or resolution of photoresists.

\section{Contributors and Collaborators}

C. Soles, W. Wu, R. Jones, V. Prabhu, C. Wang, E. Jablonski, B. Vogt, M. Wang, N. Noda, F. Starr, P. McGuiggan, D. VanderHart, C. Chiang (Polymers Division, NIST); D. Fischer, S. Sambasivan, (Ceramics Division, NIST); S. Satija, C. Glinka (NCNR, NIST); D. Goldfarb, K. Temple, A. Mahorowala, M. Angelopoulos (IBM T.J. Watson Research Center); B. Trinque, S. Burns, C. Willson (University of Texas at Austin); R. Puligadda, Y. Wang, C. Devadoss (Brewer Science) 


\section{Dielectric Measurements of Polymer Composite Films in the Microwave Range}

\begin{abstract}
Polymer composite materials with enhanced electromagnetic properties have been identified by the industry as essential for advancing miniaturization and functional performance of high-speed electronics. This project develops dielectric metrology specific to the needs of the electronics industry for embedded passive materials and devices. The current focus is on the measurement of permittivity at microwave frequencies and impedance at high $A C$ voltages. These unique measurements enable the identification of the key structural attributes that control the properties of organic resins filled with electronic modifiers.
\end{abstract}

\section{Jan Obrzut}

$\mathrm{I}_{\mathrm{d} d}^{\mathrm{n}}$ $\mathrm{n}$ recent years, a broadband technique has been developed successfully as a standard test method that enables dielectric measurements at microwave frequencies. The technique is based on the observation and theoretical analysis of the fundamental mode propagating at high frequencies in thin-film dielectrics that terminate a coaxial air-filled transmission line. This year, we applied 3D numerical simulations of the electromagnetic field to improve understanding of the measurement and enabled an extension to frequencies up to $12 \mathrm{GHz}$. The numerical simulations were used to identify and quantitatively analyze the residual inductance of the film specimens. For high-k materials with a high capacitance density, even small residual inductance can cause resonant oscillations, which leads to systematic uncertainties in the measured permittivity. Results from the numerical simulations helped formulate a closed-form expression for the input impedance of complex thin-film materials that is accurate up to $12 \mathrm{GHz}$. Broadband dielectric measurements of high-k composite materials that are of interest to bio- and nanotechnology and microelectronics are now possible and at previously inaccessible frequencies.

In partnership with IPC, we initiated a standard test method development and chaired the IPC-D-37d test method subcommittee for Embedded Passive Devices. We guided the design of the test protocol and made arrangements with co-sponsoring member companies for round robin evaluation. The committee forum has recognized NIST for being the leading laboratory in the characterization of high-k materials and for effectively addressing the standardization needs of the emerging electronic packaging technologies.

A suitable testing procedure has been demonstrated for unambiguous determination of dielectric properties at high $\mathrm{AC}$ voltages. The specimen voltage and current are determined as complex quantities from the corresponding time resolved voltage waves. The new testing procedure represents a compatible extension of the existing standard test method but is better suited for capacitive and/or resistive thin-film materials. It was found that embedded passive materials do not exhibit a constant impedance characteristic, as in conventional dielectrics, but, rather, their impedance sharply decreases with increasing voltage. This change in impedance could not be detected by the conventional test methods.

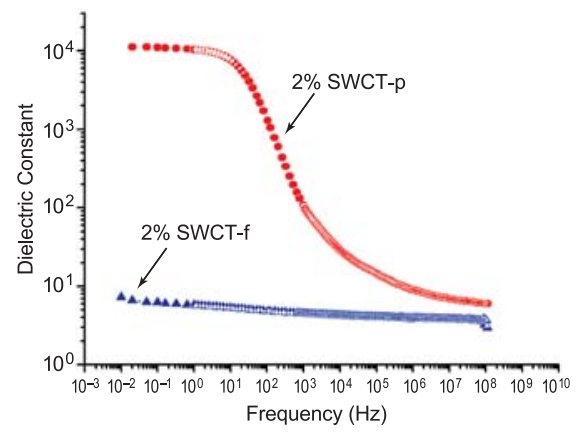

Figure 1: Dielectric properties as a function of frequency for normal and functionalized nanotubes in PMMA.

Our unique capability to measure the dielectric relaxation time in the sub-nanosecond regime and the relaxation strength for a wide range of dielectric permittivity values is used to quantify dispersion, alignment, and structure in nanostructured composite materials. Figure 1 shows the dielectric properties of two composites containing a $2 \%$ mass fraction of single wall carbon nanotubes (SWCT) dispersed in poly(methyl methacrylate) (PMMA). The SWNTs were surface-modified to affect dispersion and coupling.

Functionalized nanotubes, SWCT-f, showed an increase in the dielectric constant and can be rationalized based on the log mixing-rule law. In contrast, adding purified SWCT (SWCT-p) to PMMA increases the dielectric constant by 3 orders of magnitude. Small angle neutron scattering data indicate that this formulation exhibits a preferential coupling within the dispersed phase where a small amount of modifier causes a large enhancement in the properties of the mix. These results can be utilized in advanced engineering of plastic composites to obtain enhanced structural, optical, thermal, and electronic properties.

\section{Contributors and Collaborators}

A. Anopchenko, K. Kano, (Polymers Division, NIST); B. Rust (Mathematical and Computational Sciences, NIST); R. Croswell (Motorola); D. McGregor (DuPont); H. Wang (Michigan Technical University); K. Winey, J. Fischer (University of Pennsylvania) 


\section{Materials Structure Characterization}

Fundamental to the field of materials science and engineering is the study of the relationships between processing, structure, properties, and performance of materials. Therefore, tools and techniques for the characterization of materials structure is a cornerstone of the field. The NIST Materials Science and Engineering Laboratory (MSEL) has a long tradition of supporting and developing measurement methods and facilities for materials structure characterization. Facilities within MSEL include optical and electron microscopy, optical and electron scattering and diffraction, a state-of-the-art x-ray diffractometer, the NIST Center for Neutron Research, and experimental stations at the National Synchrotron Light Source (NSLS) at Brookhaven Laboratory and at the Advanced Photon Source (APS) at Argonne National Laboratory. At the NSLS, NIST operates a soft x-ray station in partnership with Dow and Brookhaven National Laboratory. At the APS, NIST is a partner with the University of Illinois at Urbana/Champaign, Oak Ridge National Laboratory, and UOP in a collaboration called UNICAT. At both facilities, NIST scientists and researchers from industry, universities, and government laboratories perform state-of-the-art measurements on a wide range of advanced materials. NIST scientists have consistently advanced the limits of these facilities in order to improve spatial resolution and sensitivity needed, for example, to interrogate the microstructure of both highly anisotropic and/or gradient materials such as advanced thermal coatings and fuel cell systems. Studies currently underway at these facilities include: in-situ measurements of nanoparticle production; structure and dispersion of carbon nanotubes; three-dimensional imaging of natural and artificial tissues; surface and subsurface damage in UV lithography optics; strain-induced ferroelectric transitions in thin films; and determination of molecular orientation and bond concentration on chemically heterogeneous surfaces.

The materials characterization program has a strong emphasis on electron microscopy. The MSEL Electron Microscopy Facility provides structure and compositional characterization of a wide range of materials. The facility consists of two transmission electron microscopes (TEMs), three scanning electron microscopes (SEMs), a specimen preparation laboratory, and an image analysis/computational laboratory. The JEM3010 TEM resolves the atomic structure and employs an energy selecting imaging filter and x-ray detector (EDS) for analytical characterization of thin foil specimens. The JSM6400 SEM employs electron backscattered diffraction/phase identification and EDS systems to characterize the texture and composition of materials. Highlights from the facility for FY2003 include: the computer domain is now active, providing streamlined user access and network file storage; a research collaboration with the NIST Semiconductor Electronics Division is underway to characterize quantum effects in confined Si devices; the size and shape of III-V quantum dots are characterized with the NIST Optoelectronics Division; and composition maps of electrodeposited nanowires with tunable magnetic properties are generated in collaboration with Johns Hopkins University.

This MSEL program also incorporates standards activities. A state-of-the-art x-ray diffractometer has been developed to study the metrology of powder diffraction in order to develop the next generation of diffraction standard reference materials. A variety of standard reference materials (SRMs) needed by the U.S. polymers industry, research laboratories, and other federal agencies have recently been developed: polyethylene of narrow mass distribution; nonlinear fluids for rheological measurements; melt flow standards; and the first reference biomaterial, an orthopedic grade ultra-high molecular weight polyethylene.

Recent program activities utilize matrix-assisted laser desorption/ionization time-of-flight mass spectrometry (MS) to address the need for improved characterization of the molecular structure of polyolefins, a dominant commercial polymer. This work has extended the upper mass limit detectable by MS to $15,000 \mathrm{~g} / \mathrm{mol}$ and, by observing individual oligomeric species by mass, has revealed details of the molecular structure.

A major cross-cutting activity within this program is the multi-scale, multi-modal imaging and visualization project. The goal of this project is to combine disparate sets of 3-D imaging data that contain complementary information on overlapping length scales to produce an interactive visualization scheme for multivariate data sets. This year focused on further improvements and additions to the suite of imaging tools for tissue engineering metrology. Osteoblasts cultured in a poly ( $\varepsilon$-caprolactone) scaffold have been imaged, demonstrating the advantages of optical coherence and confocal fluorescence microscopies over conventional laser scanning confocal microscopy. This instrument was upgraded by completely rebuilding the image acquisition software, hardware, and optical train. These improvements enabled an increase in image acquisition speed (2X) and accuracy (from $5 \mu \mathrm{m}$ to $360 \mathrm{~nm}$ ).

Contact: David R. Black 


\section{Coherent Anti-Stokes Raman Micro-spectroscopy ( $\mu$-CARS) for Understanding Tissue Growth in Scaffold Constructs}

\begin{abstract}
Effective and efficient regenerative medicine efforts require a thorough understanding of cell response to scaffold morphology, surface characteristics, and local biochemical conditions. We are developing a fast, high-resolution chemical microscopy technique that allows us to monitor in situ cell activity and growth along with local scaffold morphology, and relevant surface and solution chemical components in a manner that is non-invasive to delicate, rapidly dividing cells such as found in tissue scaffold constructs. This technique will allow correlations to be made between cells and environmental factors at the cellular level.
\end{abstract}

\section{Marcus T. Cicerone and Tak W. Kee}

\begin{abstract}
$A$ important approach to regenerative medicine A involves the use of tissue scaffolds as platforms for cell growth. These scaffolds serve to guide the shape and structure of the final regenerated tissue structure and often serve to provide structural support to surrounding tissues while the target tissue regenerates. These scaffolds may take the form of extruded polymer blends, microprinted or electrospun structures, or hydrogels.
\end{abstract}

A nominal aim in design of tissue scaffolds is to make the local environment of the cells conducive to their normal growth and development. Presently, the parameters for the physical and chemical design of these constructs are not well understood. Furthermore, existing volumetric imaging systems that have chemical sensitivity sufficient for tracking cell behavior, such as Magnetic Resonance Imaging (MRI) and Computed Axial Tomography (CAT), do not have the spatial resolution to track individual cells or detect local scaffold structure. A non-invasive imaging methodology that provided sub-micrometer spatial resolution, volumetric imaging, and chemical sensitivity would greatly facilitate progress in understanding cell response to tissue scaffold environment.

Coherent anti-Stokes Raman spectroscopy (CARS) is a vibrational spectroscopy, similar to infra-red (IR) or spontaneous Raman spectroscopy, and thus has potential for excellent chemical specificity. Infrared microscopy cannot be used to track single cells due to its poor spatial resolution. Raman microscopy is too inefficient, and its use leads to cell damage. CARS, on the other hand, can be used to chemically interrogate single-cells in a repetitive manner without adverse effects. Unlike IR and Raman, CARS is a multi-photon spectroscopy, requiring a pump pulse to excite molecules into a virtual electronic state and a second
(Stokes) pulse to induce relaxation into vibrationally excited states. The identity of those states is then reported by the system's response to a third pulse (pump). A schematic diagram of the CARS process is given in Figure 1.

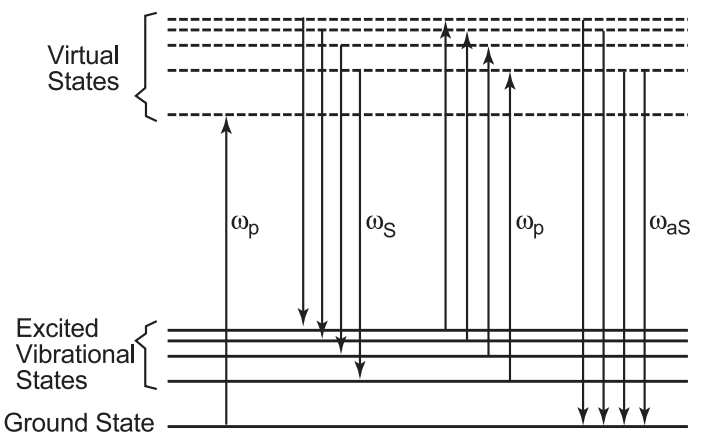

Figure 1: Schematic of CARS process. Upward arrows: absorption. Downward arrows: emission. $\omega_{p}$ : pump light. $\omega_{S}:$ Stokes light. $\omega_{a S}:$ emitted anti-Stokes light.

In order to realize the potential for chemical specificity with CARS, a full vibrational spectrum needs to be generated, requiring a broadband Stokes pulse. This has not been done before with a CARS microscope.

This year, we solved several technical problems associated with the broadband Stokes light and started building the CARS microscope. The technical problems resolved are: generation of broadband light via photonic crystal fiber, compensation for chromatic aberration so that the entire $200 \mathrm{~nm}$ band of Stokes and pulse light can be focused at the same spot to within $300 \mathrm{~nm}$, and the rapid acquisition of spectral data, which will require modification of a commercial CCD camera.

Our long-term goal for this instrument is to use it for in situ monitoring of cell growth in tissue scaffolds. This will include acquisition of broadband Raman spectra from cells, medium, and scaffold, and application of multivariate analysis (MVA) techniques for the rapid "decoding" of the spectra to obtain high-resolution chemical information. Our short-term goals include developing the MVA model for sorting out the spectra and obtaining vibrational spectra that are characteristic of known cell response to well-controlled stimuli.

\section{Contributors and Collaborators}

A. Sehgal (Polymers Division, MSEL); L. Richter (Surface and Microanalysis Science Division, CSTL); R. Tuan, W.-J. Li (NIH/NIAMS); D.M. Zhang (Purdue University); W. Wadsworth (University of Bath) 


\section{Polymer Standards}

Producers, processors and users of synthetic polymers use polymer standards for calibration of instruments, assessment of laboratory proficiency, test method development, and materials improvement. The objectives of this project are to: assure continuity of NIST Standard reference materials by recertifying standards as stock is depleted; develop a database system on the properties of biomaterials; measure material properties of polymers used in intraocular lenses for inclusion in a reference database and for producing reference biomaterials; and prepare a method for analysis of polystyrene by mass spectrometry and submit to various Standards Developing Organizations (SDO) (ASTM, DIN, ISO and JIST) for introduction into the standard test method literature.

\section{Bruno Fanconi and Charles Guttman}

$\mathrm{R}^{\mathrm{e}}$ eference Materials, RM, and Standard Reference Materials, SRM, are produced to address needs of the producers, processors, and users of synthetic polymers for calibration of instruments, assessment of laboratory proficiency, test method development, and materials development. Two melt flow rate standards have been recertified for use by producers and processors to calibrate instruments used to assess the processibility of polymer resins. A second standard polymer fluid, SRM ${ }^{\circledR} 2491$, was issued by NIST. The standard, certified for rheological properties, serves the needs of instrument manufacturers and testing laboratories as secondary standards for calibrating viscometers. Also issued in FY 2003 is $\mathrm{SRM}^{\circledR} 2888$, the first polymer molecular mass standard produced with the complete mass spectrum as ancillary data. This standard resulted from a collaborative effort among standards laboratories in Europe, the U.S., and Japan to establish a standard protocol for measurement of the mass distribution of synthetic polymers by mass spectrometry.

Producers of polyolefins are achieving property enhancements through control of branch architecture. This development has intensified the need for polymer standards certified for branch content. The ultimate solution to this need may come from development of new methods for characterizing branching, such as polymer mass spectrometry. In the near term, we exploited the wealth of characterization data in the literature of $\mathrm{SRM}^{\circledR} 1476$, a branched polyethylene that was formerly issued by NIST as a melt flow rate standard. Although the remaining supply of resin from which $\mathrm{SRM}^{\circledR} 1476$ was produced is insufficient for a melt flow standard, it is adequate for molecular standards. Among the characterization data published on $\mathrm{SRM}^{\circledR} 1476$ are results of round-robin investigations of branching characterization by chromatographic methods. This polymer, SRM ${ }^{\circledR} 1476$, has become a de facto branch standard and is referred to in the scientific literature as such. The plan is to certify the intrinsic viscosity and size by light scattering in addition to providing ancillary information on the branch content by high-resolution solution state Nuclear Magnetic Resonance (NMR).

To support the growing need for reference biomaterials, NIST released RM 8457, a medical grade ultra-high molecular weight polyethylene, UHMWPE, in the form of $0.5 \mathrm{~cm}$ cubes. The reference material supports efforts in ASTM to develop an improved test method for determination of degree of crosslinking induced by irradiation of UHMWPE. Manufacturers of medical products made of UHMWPE are researching the use of ionizing radiation to improve wear resistance. In collaboration with vendors of intraocular lens (IOL) materials, testing laboratories, the National Institutes of Health, and the LSU Medical School, data acquisition has commenced on a series of IOL polymers for the purpose of adding these to a reference material database, which is under development. The intent is to certify these IOL biomaterials as NIST reference materials to aid research and development of improved materials and for use in standards development. Active participation in ASTM committee F4 seeks to expedite introduction of reference tissue scaffolds to aid in the development of characterization methods for their structure and properties. Several NIST workshops dealing with biomaterials identified this pursuit to be of high priority.

In addition to the reference biomaterials/data activities, the group has been active in planning for polymers-related standards conferences and workshops. These have been primarily with the ASTM and include a workshop on cleanliness of medical implants (2003), planning for a symposium on cleanliness of medical implants (2005), a symposium on cell-materials signaling (2003), and a workshop on Uncertainty, Precision and Bias (2006).

\section{Contributors and Collaborators}

W. Blair, K. Flynn, J. Maurey, J. Tesk (Polymers Division, NIST); W. Guthrie, S. Leigh (Statistical Engineering Division, NIST) 


\section{Polyolefin Mass Spectrometry}

\author{
Saturated hydrocarbon polymers dominate \\ the commercial marketplace. A polymer's \\ molecular-mass distribution is critical in \\ determining its performance properties. Mass \\ spectrometry is currently the most promising \\ method for obtaining accurate absolute molecular \\ mass distributions. Here we address the difficulties \\ in forming intact, gas-phase macromolecular \\ ions of these critical materials.
}

\section{William E. Wallace}

$\mathrm{M}$ olecular mass distribution measurement by mass spectrometry (MS) requires the formation of intact macromolecular ions in the gas phase. This is typically accomplished by the gas-phase association of metal cations $\left(\mathrm{Na}^{+}, \mathrm{K}^{+}, \mathrm{Cu}^{+}, \mathrm{Ag}^{+}\right.$, etc. ) with polar or polarizable functional groups on the polymer. Before we began this project, polymers that lack such functionality (like polyethylene and polypropylene) were not amenable to mass spectrometric characterization. In the past year, we have advanced two novel methods of polyolefin macromolecular ion formation: covalent cationization and organometallic cationization.

Covalent cationization works on the principle that saturated polyolefins, regardless of the synthetic route, typically contain some fraction of unsaturated vinyl end-groups. Through ex situ preparative means, an organic cation is covalently bonded to this end-group to produce the necessary ionization for the creation of intact gas-phase macromolecules by matrix-assisted laser desorption/ionization (MALDI). After optimizing the covalent cationization chemistry, ions as high as $15,000 \mathrm{u}$ were placed in the gas phase; however, this mass limit could not be exceeded even with polyolefin materials whose extent of chemical modification had been positively verified by Nuclear Magnetic Resonance (NMR) and Fourier Transform Infrared (FTIR) spectroscopies. Furthermore, an analogous study demonstrated successful MS analysis of high-mass polystyrene $\left(M_{n} \approx 40 \mathrm{ku}\right)$ confirming the general validity of the ionization method. Confident in the utility of covalent cationization for macromolecular ion generation, our focus turned toward physical aspects of polyolefins.

The high crystallinity of linear polyethylenes may be a significant barrier to the formation of single-oligomer gas-phase ions. To overcome this barrier, a new mass spectrometer with a heated sample stage was acquired, installed, and tested. This will allow in situ heating of the polyethylene to above its melting temperature, thus eliminating crystallinity as a barrier to macromolecular ion generation.

In the area of organometallic cationization the charging agent of choice, the $\eta^{5}$-cyclopentadienyl cobalt cation $\left(\mathrm{CpCo}^{\circ+}\right)$, was shown by Fourier Transform Mass Spectrometry (FTMS), to successfully charge alkanes up to $\mathrm{C}_{60} \mathrm{H}_{122}$ (mass $843 \mathrm{u}$ ) by double-dehydrogenation with no evidence of carbon-carbon bond cleavage. Recently, the method was extended to waxes confirming the use of this ionization technique on polydisperse alkane mixtures.
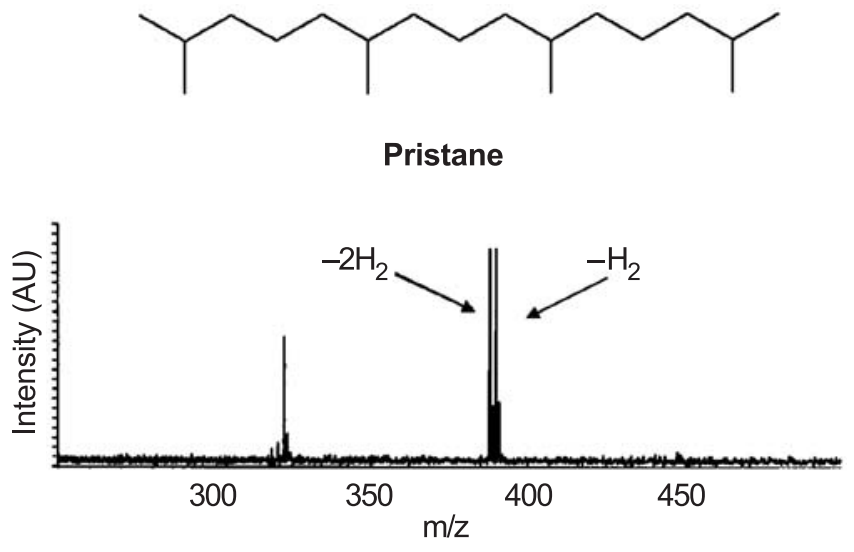

Figure 1: The FTMS spectrum above shows the gas-phase reaction of pristane with the $\mathrm{CpCo}^{\bullet+}$ ion. (AU represents arbitrary units of intensity.)

In an exciting new development, organometallic cationization was found to be branch-point sensitive. In studies of pristane (see Figure 1), both doubleand single-dehydrogenation were observed in strong contrast to linear alkanes where only the doubledehydrogenation reaction is observed. This opens the possibility for mass spectrometric studies where the number of branch points in a polyolefin may be counted on an oligomer-by-oligomer basis.

\section{Contributors and Collaborators}

B.J. Bauer, W.R. Blair, H.C.M. Byrd, B.M. Fanconi, K.M. Flynn, C.M. Guttman, S. Lin-Gibson, D.L. Vanderhart, S.J. Wetzel (Polymers Division, NIST); J.E. Girard (American University); D.R. Ridge (University of Delaware) 


\section{Nanocharacterization}

The emphasis on nanotechnology around the world and the successful implementation of the National Nanotechnology Initiative in the U.S. are accelerating the development of science and technology at the nanoscale. Nanotechnology is expected to play a key role within the next 10 years in a wide spectrum of industry sectors including manufacturing, information technology, electronics, and healthcare. Novel devices at the micro- and nanoscale will become increasingly important in all of these industries. The ability to measure dimensions, characterize materials, and elucidate structures of new and novel materials at the nanoscale will be critical to the advancement of nanotechnology. In addition, one of the exciting prospects of nanotechnology lies in the ability of molecules or particles, under specific conditions, to self-assemble to form new materials with unusual properties. Successful development of these new materials will require the ability to monitor such processes at the nanoscale in real time. Metrology, the science of measurement, is therefore the foundation of nanotechnology. Standards and reference materials will also provide essential infrastructural support to this emerging technology.

The objective of the program in the NIST Materials Science and Engineering Laboratory (MSEL) is to develop basic measurement metrology at the nanoscale for the determination of bulk and surface material properties and for process monitoring. Measurement methods are being developed for use in conjunction with new instrumentation and calibration artifacts.

The scope of the program encompasses metals, ceramics, and polymers in various forms - particles, thin films, nanotubes, and self-assembled structures — and also includes studies of nanocomposites and liquid-state properties for microfluidics-based fabrication and measurement techniques. Physical properties such as mechanical strength, elastic moduli, friction, stiction, adhesion, and fatigue strength are measured, as well as the size of nanoparticles and the structure and dispersion behavior of nanoparticulate systems. Other properties such as electrical conductivity, thermal conductivity, magnetic properties, electronic properties, and optical properties are also examined. While the program focuses on developing measurement techniques at the nanoscale, proper data interpretation requires fundamental studies in nanomechanics, scaling laws, and imaging techniques.

There are currently ten projects under the program:

- Bridging Length Scales in Theory and Modeling;

- Electrochemical Processing of Nanoscale Materials;

- Metrology for Nanoscale Properties;

- Nanofiller Processing;

- Nanoindentation;

- Nanomechanics and Standards;

- Nanoscale Manufacturing;

- Nanotribology and Surface Properties;

- Particle Metrology and Standards; and

- Physical Properties of Thin Films and Nanostructures.

In many of these individual projects, objectives are directed toward the study of a particular class of materials or material properties, but the underlying theme of the program as a whole is to develop reliable, accurate measurement techniques for a broad range of materials and material properties at the nanoscale. As just one example, four methods for the determination of the elastic properties of thin films atomic force acoustic microscopy, surface acoustic wave spectroscopy, Brillouin light scattering, and nanoindentation - are being compared using common sets of specimens. This study will lead to a better understanding of the complementary nature of these techniques for measuring nanostructured materials and their combined use to determine supplementary properties such as film thickness or density. Standard reference materials are being developed in collaboration with other National Measurement Institutes around the world for use in the verification of the performance of these instruments.

Contact: Stephen M. Hsu 


\section{Process Monitoring of Polymer Clay Nanocomposites}

Real-time, on-line measurements of polymer composite materials properties are desirable not only because they preempt the need for post-processing characterization but also because they can be used to monitor resin conditions, to detect problems with processing parameters, to control the process, and to predict performance of the final product. In this project, we have developed measurement techniques based on optical, dielectric, and rheological techniques. NIST has filled an industry need by designing and constructing process sensors and by collaborating with industry partners in the application of new sensor technology to process monitoring.

\section{Anthony J. Bur}

$\mathrm{T}^{\mathrm{s}}$ he state of microstructure that forms during the compounding of polymer and clay nanocomposites determines their performance properties. On-line, real-time measurements that are sensitive to microstructure conformation yield a database that can improve efficiency and productivity by giving processors immediate knowledge of the microstructure state. Real-time measurements can potentially minimize the extent of off-line microstructure determinations using $\mathrm{x}$-ray and Transmission Electron Microscopy (TEM) observations. The on-line instrumentation that we have developed is a multifunctional device that contains dielectric, pressure, optical, and ultrasonics sensor ports. The instrument is designed to attach to the end of a twin-screw extruder. The resin flow through the sensor conforms to a slit that is $2 \mathrm{~mm}$ high by $28 \mathrm{~mm}$ wide, and the sensors are aligned to interrogate the resin in the slit region. The extrudate, shown in the picture below, consists of a strip of material that can be cut into pieces for off-line measurements.

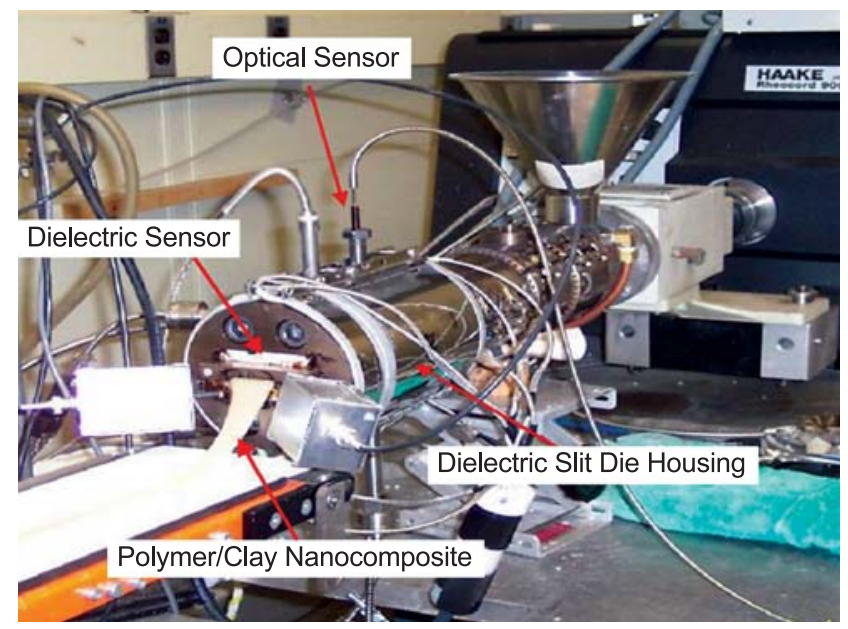

Nylon 6, when compounded with certain clays containing polar organo modifiers, can form a nanocomposite consisting of exfoliated silicate platelets that are a few nanometers thick by tens of nanometers in diameter. On the other hand, if the organo modifier is nonpolar, then the clay particle remains in its aggregate condition of a micrometer size particulate when compounded with nylon 6 . When the composite is subjected to an alternating electric field, the dielectric contrast between particle and resin forms a resistance-capacitance network that expresses a dielectric relaxation known as a Maxwell-Wagner (MW) relaxation. The characteristic relaxation time of the resin/nano-platelete combination is longer than that of the resin/aggregate because the nanosize silicate platelet has a high, effective capacitance.

The table shows characteristic relaxation times and dielectric relaxation strengths $\Delta \varepsilon$ for three compounded nylons: neat, exfoliated clay nanocomposite, and aggregate clay composite. The measurements were carried out at processing temperature, $240{ }^{\circ} \mathrm{C}$. After correcting the data for DC conductivity and electrode polarization effects, we observed only one dielectric relaxation in the neat polymer, but two relaxations were observed in the composites. The single relaxation of the neat is associated with the $\alpha$ relaxation $(\log \mathrm{f}=2.40)$ of the macromolecule, and the two relaxations of the composites are the $\alpha$ relaxation $(\log \mathrm{f}=2.70$ and 2.46$)$ and the MW relaxation at $\log \mathrm{f}=1.98$ and 1.33 for which the exfoliated microstructure displays the lowest characteristic frequency. Large $\Delta \varepsilon$ is characteristic of MW relaxation.

\begin{tabular}{|c|c|c|c|}
\hline & NY 6 neat & $\begin{array}{c}\text { NY 6/clay } \\
\text { aggregate }\end{array}$ & $\begin{array}{c}\text { NY 6/clay } \\
\text { exfoliated }\end{array}$ \\
\hline \hline Log $\mathrm{f}_{1}$ & 2.40 & 2.70 & 2.46 \\
\hline $\log \mathrm{f}_{2}$ & & 1.98 & 1.33 \\
\hline$\Delta \varepsilon_{1}$ & 441 & 1700 & 2200 \\
\hline$\Delta \varepsilon_{2}$ & & 18,200 & 18,400 \\
\hline
\end{tabular}

\section{Contributors and Collaborators}

C. Snyder, Y. Lee, N. Noda, S. Roth, V. Prabhu, P. Start, S. Hudson, J. Obrzut (Polymers Division, NIST); D. Ho, C. Glinka (NIST Center for Neutron Research); M. McBrearty (Chemical ElectroPhysics) 


\section{Nanomanufacturing}

In nanoscale manufacturing, knowledge of inherent and process-induced properties is crucial. In new realms of nanoscale manufacturing, nanomaterials will be manufactured in nanoliter volumes, combining so-called top-down and bottom-up or self-assembly approaches. Miniature tools to characterize such materials and processes are needed. We are developing devices that can impose a wide variety of process flows and measure flow-induced properties at transient and persistent conditions.

\section{Steven Hudson and Frederick Phelan, Jr.}

$\mathrm{T}$ he four-roll mill is a macroscopic flow device that has played a critical role in elucidating the interactions between flow and material response. In the microscale regime, an analogous device is needed to efficiently characterize fluids. Merely scaling down the four-roll mill to microscopic dimensions is inherently problematic due to the rotating components. Instead, we are developing a device which uses pressure-generated flow to achieve the same flow types as a four-roll mill, but is miniaturizable, and has greater control over the flow. It is also integrable into high power microscopes and scattering devices.
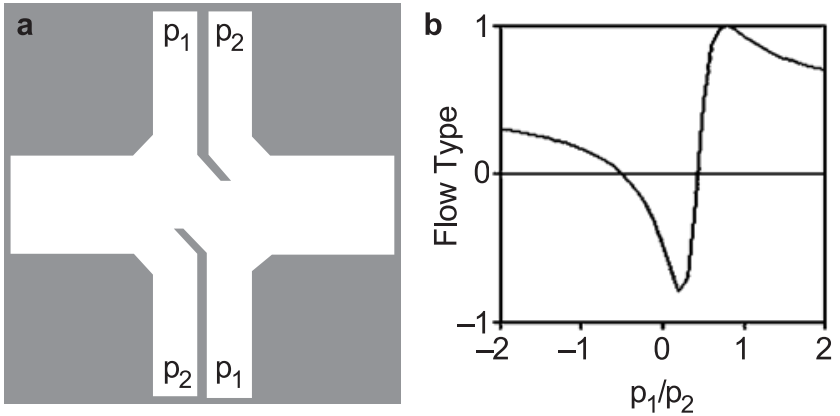

Figure 1: a) Design of a microchannel analog of the 4-roll mill. b) Flow type as a function of pressure ratio $p_{1} / p_{2}$. Flow type values equal to $-1,0$, and 1 correspond to pure rotation, shear and extension, respectively.

To evaluate various candidate geometries for a pressure-driven microchannel device, flow generated in these devices was calculated in the (Stokes) limit of low Reynolds number by the finite element method. Although a variety of geometries produce highly extensional flow, shear flow is produced at the stagnation point only when opposing channels are offset laterally. A suitable geometry is shown in Figure 1. A wide range of flow type, including pure shear, is possible in this device by adjusting the relative flow rate in the channels. The relative amount of rotation and its direction can also be adjusted. Figure 2 illustrates the flow streamlines for conditions that yield extensional and shear flow.
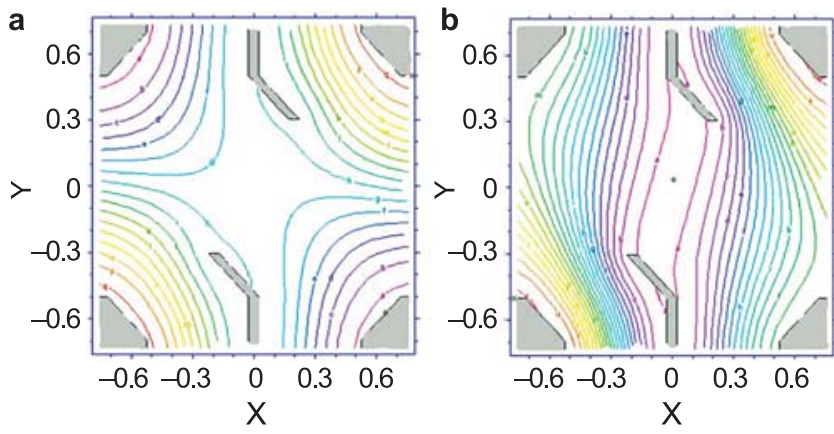

Figure 2: 2D Stokes calculations of pressure-driven flow in microchannels. Streamlines are shown for a) extensional and b) shear flow conditions.

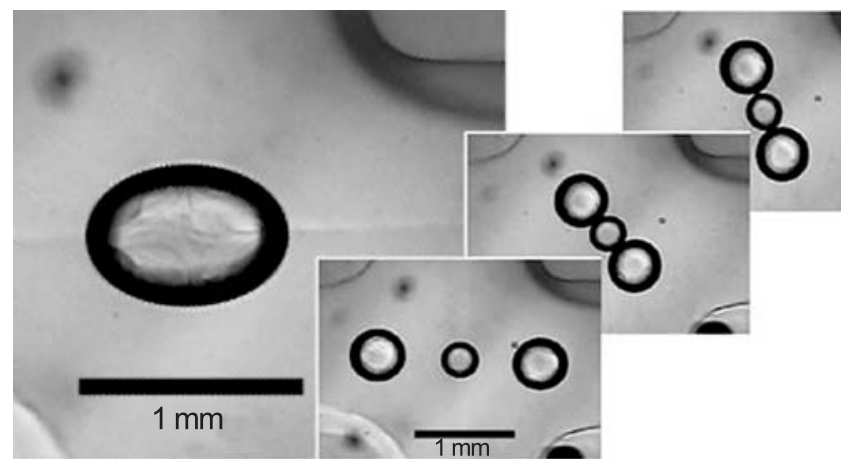

Figure 3: Images of drops in a computer-controlled cross-channel extensional flow device. Image analysis control feedback adjusts the flow to keep the drop (s) at the stable stagnation point of the device during deformation (shown at left) and collision experiments (series at right).

A computer control system, with image analysis feedback, has been developed. Experiments with immiscible polymeric fluids have demonstrated that, by using this device, drops can be moved into the device, placed at the stagnation point, deformed, and broken (Figure 3). Drops were then caused to collide and coalesce. Surface tension-driven relaxation of drop deformation has been measured. Based on these results, a focused project to measure interfacial tension, with partner companies in the NIST Combinatorial Methods Center (NCMC), was established.

\section{Contributors and Collaborators}

J.T. Cabral, J.A. Pathak (Polymers Division, NIST); NIST Combinatorial Methods Center Focused Project (with member companies) to measure interfacial tension. 



\section{Polymers Division Fy03 Annual Report Publication List}

\section{Characterization and Measurement - Internal Capabilities/Method Development}

\section{Polymer Crystallization}

J.F. Douglas, V. Ferreiro, J. Warren, and A. Karim, "Observation of Growth Pulsations in Polymer Dendritic Crystallization in PEO/PMMA Blend Films," ACS Polymer Preprints 43, 862-863 (2002).

V. Ferreiro, J.F. Douglas, J. Warren, and A. Karim, "Non-Equilibrium Pattern Formation in the Crystallization of Polymer Blend Films," Physical Review E 65, 042802:1-4 (2002).

V. Ferreiro, J.F. Douglas, J.A. Warren, and A. Karim, "Growth Pulsations in Symmetric Dendritic Crystallization in Thin Polymer Blend Films," Physical Review E 65, 051606:1-16 (2002).

D.L. VanderHart and C.R. Snyder, "Proton NMR Characterization of Room-Temperature Aging after Modest Thermal Cycling in Isotactic Polypropylene," Macromolecules 36, 4813-4826 (2003).

D.L. VanderHart, C.R. Snyder, and R.G. Alamo, "Proton NMR Characterization of Room-Temperature Aging in Isotactic polypropylene and Ethylene-Octene Copolymers," ACS Polymer Preprints 44, 285-286 (2003).

\section{Gelation, Diffusion, and the Glass Transition}

D. Bedrov, G.D. Smith, and J.F. Douglas, "Influence of Self-Assembly on Dynamical and Viscoelastic Properties of Telechelic Polymer Solutions," Europhysics Letters 59, 384-390 (2002).

J.F. Douglas, M.S. Kent, S.K. Satija, and A. Karim, "Polymer Brushes: Structure and Dynamics," in Encyclopedia of Materials: Science and Technology, (in press).

S.C. Glotzer, Y. Gebremichael, N. Lacevic, T.B. Schroder, and F.W. Starr, "Spatially Heterogeneous Dynamics in Liquids Near Their Glass Transition," in ACS Symposium Series, (Washington, D.C.: American Chemical Society, 2002) pp. 214-227.

N. Lacevic, F.W. Starr, T.B. Schroder, V.N. Novikov, and S.C. Glotzer, "Growing Correlation Length on Cooling Below the Onset of Caging in a Simulated Glass-Forming Liquid," Physical Review E 66, art. no. 030101 (2002).
S. Lin-Gibson, H.J. Walls, S.B. Kennedy, and E.R. Welsh, "Chitosan Hydrogels: Crosslink Kinetics and Gel Properties," ACS Polymeric Materials: Science and Engineering 88, 199-200 (2003).

P.A. Netz, F.W. Starr, M.C. Barbosa, and H.E. Stanley, "Relation between Structural and Dynamical Anomalies in Supercooled Water," Physica A 314, 470-476 (2002).

H.E. Stanley, S.V. Buldyrev, N. Giovambattista, E. La Nave, S. Mossa, A. Scala, F. Sciortino, F.W. Starr, and M. Yamada, "Application of Statistical Physics to Understand Static and Dynamic Anomalies in Liquid Water," Journal of Statistical Physics 110, 1039-1054 (2003).

F.W. Starr, C.A. Angell, and H.E. Stanley, "Prediction of Entropy and Dynamic Properties of Water below the Homogeneous Nucleation Temperature," Physica A - Statistical Mechanics And Its Applications 323, 51-66 (2003).

F.W. Starr, S. Sastry, J.F. Douglas, and S.C. Glotzer, "What Do We Learn from the Local Geometry of Glass-Forming Liquids?" Physical Review Letters 89, 5501-5505 (2002).

\section{Nanoscale Science, Engineering and Technology}

R.D. Davis, J.W. Gilman, and D.L. VanderHart, "Processing Degradation of Polyamide 6/Montmorillonite Clay Nanocomposites and Clay Organic Modifier," Polymer Degradation and Stability 79, 111-121 (2003).

R.C. Hedden and B.J. Bauer, "Structure and Dimensions of PAMAM/PEG Dendrimer-Star Polymers," Macromolecules 36, 1829-1835 (2003).

R.C. Hedden, B.J. Bauer, A.P. Smith, F. Gröhn, and E.J. Amis, "Templating of Inorganic Nanoparticles by PAMAM/PEG Dendrimer-Star Polymers," Polymer 43, 5473-5481 (2002).

G.A. Holmes, E. Feresenbet, and D. Raghavan, "Using Self-Assembled Monolayer Technology to Probe the Mechanical Response of the Fiber Interphase-Matrix Interphase Interface," Composite Interfaces, (in press).

G.A. Holmes, J.H. Kim, C.K. Moon, and W.G. McDonough, "Coordinated Fracture and Failure Initiation in Microcomposites," in ICCE-10, July 2003, New Orleans, LA, (in press). 
U.S. Jeng, T.L. Lin, L.Y. Wang, L.Y. Chiang, D.L. Ho, and C.C. Han, "SANS Structural Characterization of Fullerenol-Derived Star Polymers in Solutions," Applied Physics A - Materials Science \& Processing 74, S487-S489 (2002).

T. Kashiwagi, A.B. Morgan, J.M. Antonucci, M.R. VanLandingham, R.H. Harris, W.H. Awad, and J.R. Shields, "Thermal and Flammability Properties of a Silica-Poly(methylmethacrylate) Nanocomposite," Journal of Applied Polymer Science 89, 2072-2078 (2003).

J.S. Melinger, V.D. Kleiman, D. McMorrow, F. Gröhn, B.J. Bauer, and E.J. Amis, "Ultrafast Dynamics of Gold-Based Nanocomposite Materials," Journal of Physical Chemistry A 107, 3424-3431 (2003).

V. Percec, A.E. Dulcey, Y. Miura, U. Edlund, V.S.K. Balagurusamy, S.D. Hudson, H. Duan, and P.A. Heiney, "Solvophobically Driven Self-Assembly of Chiral Supramolecular Dendrimers," ACS Polymer Preprints 43, 458-459 (2002).

V. Percec, M. Glodde, T.K. Bera, Y. Miura, I. Shiyanovskaya, K.D. Singer, V.S.K. Balagurusamy, P.A. Heiney, I. Schnell, A. Rapp, H.W. Spiess, S.D. Hudson, and H. Duan, "Self-Organization of Supramolecular Helical Dendrimers Into Complex Electronic Materials," Nature 419, 384-387 (2002).

D. Raghavan, E. Feresenbet, D. Yebassa, A. Emekalam, and G. Holmes, "Dispersion of Functionalized Nanoclay Platelests in an Amine-Cured Epoxy Resin System," in Materials Research Society Symposium Proceedings, 703, v9.26.1-v9.26.8, November 2001, Boston, MA.

\section{Polymer Blends}

C.C. Han, H. Wang, K. Shimizu, H. Kim, E. Hobbie, and Z.G. Wang, "Structure Development from Simultaneous Phase Separation and Crystallization of Metallocene Polyolefin Blends," ACS Polymer Preprints 43, 856-857 (2002).

E.K. Hobbie, H.S. Jeon, H. Wang, H. Kim, D.J. Stout, and C.C. Han, "Shear-Induced Structure in Polymer Blends With Viscoelastic Asymmetry," Journal of Chemical Physics 117, 6350-6359 (2002).

A. Karim, A. Sehgal, E.J. Amis, and J.C. Meredith, "Combinatorial Mapping of Polymer Blends Phase Behavior," in Experimental Design for Combinatorial and High Throughput Materials Science, edited by J.N. Cawse (Hoboken, NJ: John Wiley \& Sons, Ltd., 2003) Chap. 5, pp. 73-88.
A. Karim, K. Yurekli, C. Meredith, E. Amis, and R. Krishnamoorti, "Combinatorial Methods for Polymer Materials Science: Phase Behavior of Nanocomposite Blend Films," Polymer Engineering and Science 42, 1836-1840 (2002).

F.A. Landis, R.B. Moore, K.A. Page, and C.C. Han, "SAXS Analysis of the Thermal Relaxation Behavior of Oriented Perfluorosulfonate Ionomer Membranes," ACS Polymeric Materials: Science and Engineering 87, 121-122 (2002).

G. Matsuba, K. Shimizu, H. Wang, Z.G. Wang, and C.C. Han, "Correlation Between Phase Separation and Crystallization in Poly(ethylene-co-hexene) and Poly(ethylene-co-octene)," ACS Polymeric Materials: Science and Engineering 87, 317-318 (2002).

J. Parkas, M. Paulsson, D.L. VanderHart, and U. Westermark, "Accelerated Light-Induced Aging of Alpha-, Beta-, and Gamma-C-13-Enriched Cell Wall-Dehydrogenation Polymers Studied With Solid State C-13 NMR Spectroscopy," Journal of Wood Chemistry and Technology 22, 199-218 (2002).

K. Shimizu, H. Wang, Z. Wang, H. Kim, and C.C. Han, "Morphological Development in Copolymer Blends of Poly(ethylene-co-hexene) and Poly(ethyleneco-butene)," ACS Polymer Preprints 43, 273-274 (2002).

K. Shimizu, H. Wang, Z.G. Wang, G. Matsuba, and C.C. Han, "Phase Behavior in Polyolefin Blend," ACS Polymeric Materials: Science and Engineering 87, 319-320 (2002).

L.P. Sung, J.F. Douglas, C.C. Han, and A. Karim, "Suppression of Phase Separation Pattern Formation in Blend Films with Block Copolymer Compatibilizer," Journal of Polymer Science Part B-Polymer Physics 41, 1697-1700 (2003).

R.T. Tucker, C.C. Han, A.V. Dobrynin, and R.A. Weiss, "Small-angle Neutron Scattering Analysis of Blends with Very Strong Intermolecular Interactions: Polyamide/ionomer Blends," Macromolecules 36, 4404-4410 (2003).

Z. Wang, E. Balizer, B. Hsiao, and C.C. Han, "Structure Evolution During Ferroelectric Phase Transition in a Vinylidene Fluoride/Trifluoroethylene Copolymer," ACS Polymeric Materials: Science and Engineering 43, 203-204 (2002).

Z. Wang, H. Wang, B.S. Hsiao, D. Gersappe, and C.C. Han, "In-situ Small Angle X-ray Scattering of Crystallization in Poly(ethylene/hexene) Copolymer Subjected to Step Shear," ACS Polymer Preprints 43, 271-272 (2002). 


\section{Polymer Composites}

M.Y.M. Chiang and M. Fernandez-Garcia, "Relation of Swelling and Tg Depression to the Apparent Free Volume of a Particle-Filled Epoxy-based Adhesive," Journal of Applied Polymer Science, (in press).

M.Y.M. Chiang and J. He, "An Analytical Assessment of Using the Iosipescu Shear Test for Hybrid Composites," Composite Part B: Engineering 33, 461-470 (2002).

M.Y.M. Chiang, C.R. Schultheisz, and J. He, "The Effects of Local Inhomogeneity on Shear Property Measurements of Hybrid Composites," in SAMPE 2002 - Winter Technical Meeting, November 2002, Baltimore, MD.

E. Feresenbet, D. Raghavan, and G.A. Holmes, "Application of Self Assembled Technology to Probe Fiber-Matrix Adhesion," in Materials Research Society Symposium Proceedings, November 2001, Boston, MA.

E. Feresenbet, G.A. Holmes, and D. Raghavan, "The Influence of Silane Coupling Agent Composition on the Surface Characterization of Fiber and on Fiber-Matrix Interfacial Shear Strength," Journal of Adhesion 79, 643-665 (2003).

J. He, M.Y. Chiang, D.L. Hunston, and C.C. Han, "Application of the V-notch Shear Test for Unidirectional Hybrid Composites," Journal of Composite Materials 36, 2653-2666 (2002).

J. He, M.Y.M. Chiang, W.G. McDonough, and D.L. Hunston, "Experimental and Theoretical Evaluations of the Iosipescu Shear Test for Hybrid Fiber Composites," in SAMPE 2002, 154-164, May 2002, Long Beach, CA.

G.A. Holmes and W.G. McDonough, "E-Glass/DGEBA/m-PDA Model Composites: Time Dependent Failure in a Brittle Multi-Fiber Composite," 47th International SAMPE Symposium and Exhibition 2002, 47:2/2, 1690-1702.

G.A. Holmes, R.C. Peterson, D.L. Hunston, and W.G. McDonough, "E-Glass/DGEBA/m-PDA Single Fiber Composites: Interface Debonding During Fiber Fracture," Polymer Composites, (in press).

D.L. Hunston and W.G. McDonough, "Stiffness and Failure Behavior of Model Hybrid Composites," in Proceedings of the American Society for Composites Seventeenth Technical Conference, October 2002, West Lafayette, IN.

D.L. Hunston, Z. Miyagi, C.R. Schultheisz, S. Zaghi, and H. Binson, "Sandwich Bending Specimen for Characterizing Adhesive Properties," Mechanics of Time-Dependent Materials, (in press).
J.L. Lenhart, J.H. van Zanten, J.P. Dunkers, and R.S. Parnas, "Using a Localized Fluorescent Dye to Probe the Glass/Resin Interphase," Polymer Composites 23, 555-563 (2002).

J.L. Lenhart, J.H. van Zanten, J.P. Dunkers, and R.S. Parnas, "Characterization of Sizing Layers and Buried Polymer/Sizing/Substrate Interfacial Regions Using a Localized Fluorescent Probe," Journal of Colloid and Interface Science 257, 398-407 (2003).

S. Lin-Gibson, G. Schmidt, H. Kim, C.C. Han, and E.K. Hobbie, "Shear-Induced Mesostructure in Nanoplatelet-Polymer Gels," Journal of Chemical Physics, (in press).

S. Lin-Gibson and J.S. Riffle, "Chemistry and Properties of Phenolic Resins and Networks," in Synthetic Methods in Step-Growth Polymers, edited by M.E. Rogers and T.E. Long (New York: John Wiley \& Sons, Inc., 2003) Chap. 7, pp. 369-423.

D. Raghavan, J. He, D.L. Hunston, and D. Hoffman, "Strain Rate Dependence of Fracture in a RubberToughened Epoxy System," Journal of Adhesion 78, 723-739 (2002).

M.J. Rich, L.T. Drzal, D.L. Hunston, G.A. Holmes, and W.G. McDonough, "Round Robin Assessment of the Single Fiber Fragmentation Test," in Proceedings of the American Society for Composites 17th Technical Conference, 1-9 October 2002, West Lafayette, IN.

J. Toke, J.D. Muzzy, and M.T. Cicerone, "Using LSM to Investigate Maleated Polypropylene in Polypropylene/Glass Bead Composites," in Proceedings of the Annual Technical Meeting of the Society of Plastics Engineers, 2127-2130, May 2003, Nashville, TN.

D.L. Woerdeman, W. Ou, J.L. Lenhart, and R.S. Parnas, "Interpreting The Signal From A Localized Fluorescence Sensor: A Study By Angle-Resolved XPS And Dynamic SIMS," Journal of Colloid and Interface Science 262, 594-602 (2003).

\section{Sum Frequency Generation Spectroscopy}

K. Efimenko, W.E. Wallace, and J. Genzer, "Surface Modification of Sylgard-184 Poly(dimethyl siloxane) Networks by Ultraviolet and Ultraviolet/Ozone Treatment," Journal of Colloid and Interface Science 254, 6-315 (2002).

P.T. Wilson, K.A. Briggman, J.C. Stephenson, W.E. Wallace, and L.J. Richter, "Molecular Order at Polymer Interfaces Measured by Broad-Bandwidth Vibrationally-Resolved Sum-Frequency Generation Spectroscopy," in Quantum Electronics and Laser Science Conference (QELS 2001), 57, 189, (2001). 
P.T. Wilson, L.J. Richter, W.E. Wallace, K.A. Briggman, and J.C. Stephenson, "Correlation of Molecular Orientation with Adhesion at Polystyrene/Solid Interfaces," Chemical Physics Letters 363, 161-168 (2002).

P.T. Wilson, L.J. Richter, W.E. Wallace, K.A. Briggman, and J.C. Stephenson, "Correlation of Molecular Orientation With Adhesion at Polystyrene/Solid Interfaces," Chemical Physics Letters 363, 161-168 (2002).

\section{Characterization and Measurement - Standards and Data}

\section{Polymer Standards}

B.M. Fanconi, J.A. Tesk, and W.F. Guthrie, "Reference Material 8457, Ultra High Molecular Weight Polyethylene $0.5 \mathrm{~cm}$ Cubes," in Society of Biomaterials 29th Annual Meeting Transactions, 587, April 2003, Reno, NV.

C.M. Guttman and W.R. Blair, "Recertification of SRM 1483a, a Polyethylene," NIST SP, (in press).

C.M. Guttman, W.R. Blair, B.M. Fanconi, R.J. Goldschmidt, S.J. Wetzel, and D.L.VanderHart, "Certification of a Polystyrene Synthetic Polymer, SRM 2888," NIST SP 260-152, (in press).

J.M. Maurey, K.M. Flynn, and C.M. Guttman, "Certification of Standard Reference Material 1474a, A Polyethylene Resin,” NIST SP 260-148 (2003).

J.A. Tesk, "Reference Materials for Tissue Engineered Medical Products," in Tissue Engineering, (in press).

\section{Mass Spectrometry}

B.J. Bauer and K.M. Flynn, "MALDI of Structured Polymer Particles," in Proc. of the 51st ASMS Conference on Mass Spectrometry and Allied Topics, June 2003, Montreal, Canada, (in press).

H.C.M. Byrd, C.M. Guttman, and D.P. Ridge, "Molecular Mass Determination of Saturated Hydrocarbons: Reactivity of $\eta^{5}$ - Cyclopentadienylcobalt Ion $\left(\mathrm{CpCo}^{+}\right)$and Linear Alkanes up to C-30," Journal of the American Society for Mass Spectrometry 14, 51-57 (2003).

H.C.M. Byrd, S. Lin-Gibson, S. Bencherif, D.L. VanderHart, K.L. Beers, B.J. Bauer, B.M. Fanconi, C.M. Guttman, and W.E. Wallace, "MALDI-TOF MS Characterization of Covalently-Cationized Polymers," ACS Polymeric Materials: Science and Engineering 88, 80-81 (2003).
J.E. Girard, M. Konaklieva, J. Gu, C.M. Guttman, and S.J. Wetzel, "Characterization of Linear and Branched Polyethylenimine (PEI) by ESI-MS and MALDI-TOF-MS," in Proc. of the 51st ASMS Conference on Mass Spectrometry and Allied Products, June 2003, Montreal, Canada, (in press).

C.M. Guttman, "Mass Spectrometry," Encyclopedia of Polymer Science and Technology, (New York: John Wiley \& Sons, Inc., 2002).

C.M. Guttman, B.J. Bauer, K.L. Beers, S.A. Bencherif, H.C.M. Byrd, and S. Lin-Gibson, "MALDI-TOF MS Analysis of Chain-End Functionalized Polymers," in Proc. of the 51st ASMS Conference on Mass Spectrometry and Allied Topics, June 2003, Montreal, Canada, (in press).

A.J. Kearsley, W.E. Wallace, J. Bernal, and C.M. Guttman, "Automated Peak Picking and Integration Algorithm for Mass Spectral Data," in Proc. of the 51st ASMS Conference on Mass Spectrometry and Allied Topics, June 2003, Montreal, Canada, (in press).

S. Lin-Gibson, S. Bencherif, K.L. Beers, and H.C.M. Byrd, "MALDI-TOF-Mass Spectral Characterization of Covalently Cationized Polystyrene," Macromolecules 36, 4669-4671 (2003).

S. Lin-Gibson, L. Brunner, D.L. VanderHart, B.J. Bauer, B.M. Fanconi, C.M. Guttman, and W.E. Wallace, "MALDI MS Characterization of Cationized Covalent Polyethylene," ACS Polymer Preprints 43, 1331-1332 (2002).

S. Lin-Gibson, L. Brunner, D.L. VanderHart, B.J. Bauer, B.M. Fanconi, C.M. Guttman, and W.E. Wallace, "Optimizing the Covalent Cationization Method for the Mass Spectrometry of Polyolefins," Macromolecules 35, 7149-7156 (2002).

M.R. Nyden, W.E. Wallace, and W.H. Awad, "An Application of MALDI-MS and Reactive Molecular Dynamics in Determining the Mechanisms of Char-Formation During the Thermal and Oxidative Degradation of Polymers," in ACS Polymeric Materials: Science and Engineering, 88, 178-179, March 2003, New Orleans, LA.

W.E. Wallace and D.A. Fischer, "Resonant Soft X-ray Photofragmentation of Propane," Journal of Electron Spectroscopy and Related Phenomena 130, 1-6 (2003).

W.E. Wallace, C.M. Guttman, and S.D. Hanton, "Quantitative Synthetic Polymer Mass Spectrometry Workshop," Journal of Research of the National Institute of Standards and Technology 108, 79-85 (2003). 
S.J. Wetzel, C.M. Guttman, and J.E. Girard, "The Influence of Laser Energy and Matrix of MALDI on the Molecular Mass Distribution of Poly(ethylene glycol)," in ACS Polymeric Materials: Science and Engineering 88, 74-75 (2003).

S.J. Wetzel, C.M. Guttman, and J.E. Girard, "A Study of the Effects of Matrix and Laser Energy on the Molecular Mass Distribution of Synthetic Polymers," in Proceedings of the 51st ASMA Conference on Mass Spectrometry and Allied Products, June 2003, Montreal, Canada, (in press).

T. Yalcin, W.E. Wallace, C.M. Guttman, and L. Li, "Metal Powder Substrate Assisted Laser Desorption Mass Spectrometry for Polyethylene Analysis," Analytical Chemistry 74, 4750-4756 (2002).

\section{Electronics Materials}

\section{Polymers for Lithography}

R.L. Jones, T. Hu, E.K. Lin, W.L. Wu, D.M. Casa, N.G. Orji, T.V. Vorburger, P.J. Bolton, and G.G. Barclay, "Sub-nanometer Wavelength Metrology of Lithographically Prepared Structures: A Comparison of Neutron and X-ray Scattering," in Proceedings of the SPIE: Metrology, Inspection, and Process Control for Microlithography XVII, February 2003, Santa Clara, CA.

R.L. Jones, T.J. Hu, V.M. Prabhu, C.L. Soles, E.K. Lin, W.L. Wu, D.L. Goldfarb, and M. Angelopoulos, "Form of Deprotection in Chemically Amplified Resists," in Proceedings of the International Conference on Characterization and Metrology for ULSI Technology, March 2003, Austin, TX, (in press).

R.L. Jones, T.J. Hu, V.M. Prabhu, C.L. Soles, E.K. Lin, W.L. Wu, D.L. Goldfarb, M. Angelopoulos, B.C. Trinque, and C.G. Willson, "Deprotection Volume Characteristics and Line Edge Morphology in Chemically Amplified Resists," in Proceedings of the SPIE: Advances in Resist Technology and Processing XX, 5039, 1031, March 2003, Austin, TX.

R.L. Jones, V.M. Prabhu, D.L. Goldfarb, E.K. Lin, C.L. Soles, J.L. Lenhart, W.L. Wu, and M. Angelopoulos, "Correlation of the Reaction Front with Roughness in Chemically Amplified Photoresists," in Symposia of the American Chemical Society, (Washington, D.C.: American Chemical Society), (in press).

J.L. Lenhart, R.L. Jones, E.K. Lin, C.L. Soles, W.L. Wu, D.A. Fischer, S. Sambasivan, D.L. Goldfarb, and M. Angelopoulos, "Probing Surface and Bulk Chemistry in Resist Films Using Near Edge X-Ray Absorption Fine Structure," Journal of Vacuum Science \& Technology B 20, 2920-2926 (2002).
V.M. Prabhu, R.L. Jones, E.K. Lin, C.L. Soles, W.L. Wu, D.L. Goldfarb, and M. Angelopoulos, "Polyelectrolyte Effects in Model Photoresis Developer Solutions: Roles of Base Concentration and Added Salts," in Proceedings of the SPIE, Advances in Resist Technology and Processing XX, 5039, 404, February 2003, Santa Clara, CA.

V.M. Prabhu, R.L. Jones, E.K. Lin, and W.L.Wu, "Polyelectrolyte Effects in Model Photoresist Developer Solutions," Journal of Vacuum Science and Technology B 21, 1403-1410 (2003).

C.L. Soles, J.F. Douglas, E.K. Lin, J.L. Lenhart, R.L. Jones, W.L.Wu, D. Goldfarb, and M. Angelopoulos, "Incoherent Neutron Scattering and the Dynamics of Thin Film Photoresist Polymers," Journal of Applied Physics 93, 1978-1986 (2003).

\section{Porous Low-k Dielectric Thin Films}

B.J. Bauer, H.J. Lee, R.C. Hedden, C.L. Soles, D.W. Liu, and W.L. Wu, "SANS Characterization of Nanoporous Thin Films for the Next Generation of Integrated Circuits," ACNS WEBsite:

http://www.sns.gov/acns/, June 2002, Knoxville, TN.

R.C. Hedden, H.J. Lee, B.J. Bauer, C.L. Soles, W.L.Wu, and E.K. Lin, "Measurement of Pore Size and Matrix Characteristics in Low-k Dielectrics by Neutron Contrast Variation," in Proceedings of the International Conference on Characterization and Metrology for ULSI Technology, March 2003, Austin, TX.

H.J. Kim, W.L. Wu, B.J. Bauer, E.K. Lin, J.Y. Kim, Y.H. Kim, and H.J. Lee, "Structural Characterization of Porous Low-k SiOC Thin Films Using X-Ray Porosimetry," in IEEE International Conference on Interconnect Technology, 54-56, 2002.

H.J. Lee, E.K. Lin, B.J. Bauer, W.L. Wu, B.K. Hwang, and W.D. Gray, "Characterization of Chemical-Vapor-Deposited Low-K Thin Films Using X-Ray Porosimetry," Applied Physics Letters 82, 1084-1086 (2003).

H.J. Lee, C.L. Soles, D.W. Liu, B.J. Bauer, and W.L. Wu, "Pore Size Distributions in Low-k Dielectric Thin Films from X-ray Porosimetry," Journal of Polymer Science Part B - Polymer Physics 40, 2170-2177 (2002).

E.K. Lin, H.J. Lee, B.J. Bauer, H. Wang, J.T. Wetzel, and W.L. Wu, "Structure and Property Characterization of Low-k Dielectric Porous Thin Films Determined by X-ray Reflectivity and Small-angle Neutron Scattering," in Low Dielectric Constant Materials for IC Applications, edited by P.S. Ho, J. Leu, and W.W. Lee (Springer Publishing, Inc.), (in press). 


\section{Dielectric Characterization/Electronic Packaging}

E. Balizer, F.B. Bateman, A.S. DeReggi, and D.A. Neumann, "Electrostriction Mechanism in Electro-Irradiated Ferroelectric Copolymer: a Frequency Response Model," in 2000 Conference on Electrical Insulation and Dielectric Phenomena, 191-194.

T. Bergstresser, R. Hilburn, J. Obrzut, and K. Phillips, "Embedded Capacitance Materials and Their Application in High Speed Design," in IPC Expo Technical Conference, S06: 3.1-3.8, March 2003, Long Beach, CA.

C.K. Chiang, "The Bromine Doping of Polyacetylene," Physica 321, 139-151 (2003).

C.K. Chiang and R. Popielarz, "Polymer Composites With High Dielectric Constant," Ferroelectrics 275, 1-9 (2002).

D. Josell, W.E. Wallace, J.A. Warren, D. Wheeler, and A.C. Powell, "Misaligned Flip-Chip Solder Joints: Prediction and Experimental Determination of Force-Displacement Curves," Journal of Electronic Packaging 124, 227-233 (2002).

J. Obrzut and A. Anopchenko, "Numerical Analysis of a Coaxial Line Terminated with a Complex Gap Capacitance," in IEEE Conference on Instrumentation and Measurements, 1074-1077, May 2003, Vail, CO.

W. Sakai and C.K. Chiang, "Dielectric Properties of Multi-layer High-k Composite Films," in Materials Research Society Symposium Proceedings, 710, DD6.17.1-5, November 2002, Boston, MA.

\section{Polymer Thin Film Fundamentals}

E.Q. Chen, Y. Xia, M.J. Graham, M.D. Foster, Y.L. Mi, W.L. Wu, and S.Z.D. Cheng, "Glass Transition Behavior of Polystyrene Blocks in the Cores of Collapsed Dry Micelles Tethered by Poly(Dimethylsiloxane) Coronae in a PS-B-PDMS Diblock Copolymer," Chemistry of Materials 15, 2129-2135 (2003).

C.K. Chiang and W. Sakai, "Application of a Buffer Layer for the Dielectric Measurement of Thin Polymer Films," in Materials Research Society Symposium Proceedings, 699, R7.1.1-4, November 2002, Boston, MA.

R.L. Jones, C.L. Soles, F.W. Starr, E.K. Lin, J.L. Lenhart, W.L. Wu, D.L. Goldfarb, and M. Angelopolous, "Chain Conformation in Ultrathin Polymer Films," in Proceedings of the SPIE: Advances in Resist Technology and Processing XIX, 4690, 342-350, March 2002, Santa Clara, CA.
J.L. Lenhart and W.L. Wu, "Influence of Cross-link Density on the Thermal Properties of Thin Polymer Network Films," Langmuir 19, 4863-4865 (2003).

C.L. Soles, J.F. Douglas, W.L. Wu, and R.M. Dimeo, "Incoherent Neutron Scattering as a Probe of the Dynamics in Molecularly Thin Polymer Films," Macromolecules 36, 373-379 (2003).

C.L. Soles, J.F. Douglas, W.L. Wu, H. Peng, and D.W. Gidley, "A Broad Perspective on the Dynamics of Highly Confined Polymer Films," in Materials Research Society Symposium Proceedings, 2002.

B.D. Vogt, C.L. Soles, H.J. Lee, E.K. Lin, and W.L. Wu, "Confinement Effects on Moisture Absorption Kinetics in Polyelectrolyte Films," in ACS Polymeric Materials: Science and Engineering, September 2003, New York, NY, (in press).

C.Y. Wang, V.M. Prabhu, C.L. Soles, B.D. Vogt, W.L. Wu, E.K. Lin, and S.K. Satija, "Interdiffusion in Polystyrene and End-Functional Polystyrene Thin Films near a Solid Surface," in ACS Polymeric Materials: Science and Engineering, New York, NY, September 2003, (in press).

C.C. White, W.L. Wu, Y. Pu, M. Rafailovich, and J. Sokolov, "Probe Segregation and $\mathrm{T}_{\mathrm{g}}$ Determination of a Supported Ultra-Thin Polystyrene Film Studied by X-ray and Neutron Reflectivity, and SIMS," Polymer Engineering and Science 43, 1241-1249 (2003).

\section{Biomaterials}

\section{Tissue Engineeering Metrology}

O.L. Bailey, N.R. Washburn, and F.W. Wang, "The Use of Real-Time Polymerase Chain Reaction in Quantifying Inflammatory Responses to Biomaterials," in Society of Biomaterials 29th Annual Meeting Transactions, 513, April 2003, Reno, NV.

S.B. Kennedy, Y. Mei, R. Gross, N.R. Washburn, and E.J. Amis, "Quantifying Cell-Response to Materials Through Population Analyses Enabled by High-Throughput Techniques," in Society of Biomaterials 29th Annual Meeting Transactions, 26, April 2003, Reno, NV.

C.A. Khatri and F.W. Wang, "Controlled Release of Transforming Growth Factor from Composite Bone Grafts Consisting of Calcium Phosphate Cement and Biodegradable Particulates," in Society of Biomaterials 29th Annual Meeting Transactions, 476, April 2003, Reno, NV. 
F.W. Wang, C.A. Khatri, J.F. Hsii, S. Hirayama, and S. Takagi, "Polymer-Filled Calcium Phosphate Cement: Mechanical Properties and Controlled Release of Growth Factor," in Biomedical Engineering: Recent Developments, edited by J. Vossoughi, (in press).

N.R. Washburn, S.B. Kennedy, C.G. Simon, K.M. Yamada, and E.J. Amis, "High-Throughput Investigation of Cell Proliferation on Crystalline Polymers," in Society of Biomaterials 29th Annual Meeting Transactions, 528, April 2003, Reno, NV.

S. Yoneda, W.F. Guthrie, D.S. Bright, C.A. Khatri, and F.W. Wang, "Effects of Hydrolytic Degradation on in Vitro Biocompatibility of Poly(D,L-Lactic Acid)," in IADR/AADR/CADR 80th General Session, 1364, March 2002, San Diego, CA.

\section{Dental Materials}

J.M. Antonucci, S. Dickens, B.O. Fowler, H.H.K. Xu, and W.G. McDonough, "Chemistry of Silanes: Interfaces in Dental Polymers and Composites," in Proceedings of the Academy of Dental Materials Annual Meeting, October 2003, Charleston, SC, (in press).

J.M. Antonucci, B.O. Fowler, S.H. Dickens, and N.D. Richards, "Novel Dental Resins from Trialkoxysilanes and Dental Monomers by in-situ Formation of Oligomeric Silyl Ethers and Silsesquioxanes," ACS Polymer Preprints 43, 633-634 (2002).

J.M. Antonucci, W.G. McDonough, D.W. Liu, and D. Skrtic, "Effect of Polymer Matrices on Methacrylate Conversion and Mechanical Strength of Bioactic Composites Based on Amorphous Calcium Phosphate," ACS Polymer Preprints 43, 741-742 (2002).

J.M. Antonucci, W.G. McDonough, D.W. Liu, and D. Skrtic, "Effect of Acidic Comonomers on Methacrylate Conversion and Mechancial Strength of Bioactive Composites Based on Amorphous Calcium Phosphate," ACS Polymeric Materials: Science and Engineering 88, 50-51 (2003).

S.H. Dickens, J.M. Antonucci, and B.O. Fowler, "Evaluation of the Interactions of a Silane Agent with Dental Monomers by Near Infrared Spectroscopy," ACS Polymer Preprints 43, 747-748 (2002).

C.C. Khatri, J.W. Stansbury, C.R. Schultheisz, and J.M. Antonucci, "Synthesis, Characterization and Evaluation of Urethane Derivatives of Bis-GMA," Dental Materials, (in press).
W.G. McDonough, J.M. Antonucci, J.P. Dunkers, and G.E. Schumacher, "Glass-fiber/resin Interfacial Strength: The Microbond Test and Chemical Evaluation," in Proceedings of the Academy of Dental Materials Annual meeting, October 2003, Charleston, SC, (in press).

D. Skrtic and J.M. Antonucci, "Effect of Bifunctional Co-monomers on Mechanical Strength and Water Sorption of Amorphous Calcium Phosphate and Silanized Glass-filled Bis-GMA-based Composites," Biomaterials 24, 2881-2888 (2003).

D. Skrtic, J.M. Antonucci, and E.D. Eanes, "Amorphous Calcium Phosphate-Based Bioactive Polymeric Composites for Mineralized Tissue Regeneration," Journal of Research of the National Institute of Standards and Technology 108, 167-182 (2003).

D. Skrtic, J.W. Stansbury, and J.M. Antonucci, "Volumetric Contraction and Methacrylate Conversion in Photopolymerized Amorphous Calcium Phosphate/Methacrylate Composites," Biomaterials 24, 2443-2449 (2003).

M.D. Weir, N.D. Richards, and J.M. Antonucci, "Structural Effects of Experimental Comonomers on Conversion and Polymerization Shrinkage of Dental Composites," in Society for Biomaterials 29th Annual Meeting Transactions, 543, April 2003, Reno, NV.

H.H.K. Xu, F.C. Eichmiller, D.T. Smith, G.E. Schumacher, A.A. Giuseppetti, and J.M. Antonucci, "Effect of Thermal Cycling on Whisker-Reinforced Dental Resin Composites," Journal of Materials Science-Materials in Medicine 13, 875-883 (2002).

\section{Characterization}

G. Caliskan, A. Kisliuk, A.M. Tsai, C.L. Soles, and A.P. Sokolov, "Protein Dynamics in Viscous Solvents," Journal of Chemical Physics 118, 4230-4236 (2003).

S. Lin-Gibson, H.J. Walls, S.B. Kennedy, and E.R. Welsh, "Reaction Kinetics and Gel Properties of Blocked Diisocyanate Cross-Linked Chitosan Hydrogels," Carbohydrate Polymers, (in press).

F.W. Wang and R.E. Lowry, "Wavelength-shift Fluorescence Probes for Cure Monitoring of Resins," in Society for Biomaterials 29th Annual Meeting Transactions, 340, April 2003, Reno, NV. 


\section{Processing Characterization}

\section{Complex Polymer Flow and Instabilities}

A.J. Bur, S.C. Roth, D.W. Baugh, K.A. Koppi, M.A. Spalding, J. Gunderson, and W.C. Buzanowski, "Measuring Resin Temperature During Extrusion Using a Fluorescence Technique," in Proceedings of the Annual Technical Meeting of the Society of Plastics Engineers, 3341-3345, May 2003, Nashville, TN.

S.D. Hudson, "Wall Migration and Shear-Induced Diffusion of Fluid Droplets in Emulsions," Physics of Fluids 15, 1106-1113 (2003).

S.D. Hudson, A.M. Jamieson, and B. Burkhart, "The Effect of Surfactant on the Efficiency of Shear-Induced Drop Coalescence," Journal of Colloid and Interface Science, (in press).

S.B. Kharchenko, K.B. Migler, and P.M. McGuiggan, "Flow Induced Coating of Polymer Processing Additives: Development of Frustrated Total Internal Reflection Imaging," in Proceedings of the Annual Technical Meeting of the Society of Plastics Engineers, 2689-2693, May 2003, Nashville, TN.

M.M. Malwitz, S. Lin-Gibson, E.K. Hobbie, P.D. Butler, and G. Schmidt, "Orientation of Platelets in Multilayered Nanocomposite Polymer Films," Journal of Polymer Science: Polymer Physics Edition (in press).

K.B. Migler, Y. Son, F. Qiao, and K.M. Flynn, "Extensional Deformation, Cohesive Failure, and Boundary Conditions During Sharkskin Melt Fracture," Journal of Rheology 46, 383-400 (2002).

J.A. Pathak, M.C. Davis, S.D. Hudson, and K.B. Migler, "Layered Droplet Microstructures in Sheared Emulsions: Finite-Size Effects," Journal of Colloid and Interface Science 255, 391-402 (2002).

J.A. Pathak, S.D. Hudson, and K.B. Migler, "Structure Formation in Micro-Confined Polymeric Emulsions," ACS Polymer Preprints 43, 833-834 (2002).

Y. Son and K.B. Migler, "Cavitation of Polyethylene During Extrusion Processing Instabilities," Journal of Polymer Science: Part B Polymer Physics 40, 2791-2799 (2002).

Y. Son and K.B. Migler, "Observation of the Cavitation Phenomena Upon the Gross Melt Fracture Regime In LLDPE Extrusion," in Proceedings of the Society of Plastics Engineers 2002 Annual Technical Conference, May 20, 2002, San Francisco, CA.
M.A. Spalding, D.W. Baugh, K.A. Koppi, W.C. Buzanowski, A.J. Bur, and S.C. Roth, "Temperature Gradients in the Channels of a Single-Screw Extruder," in Proceedings of the Annual Technical Meeting of the Society of Plastics Engineers, 202-206, May 2003, Nashville, TN.

\section{Nanoscale Manufacturing}

P.M. McGuiggan and D.J. Yarusso, "Measuring the Mechanical Properties of a Thin Polymeric Film by AFM," ACS Polymeric Materials: Science and Engineering 88, 532 (2003).

J.A. Pathak and K.B. Migler, "Droplet-String Deformation and Stability During Micro-Confined Shear Flow," Langmuir, (in press).

J.A. Pathak, E. Robertson, S.D. Hudson, and K.B. Migler, "Droplet Microstructure and String Stability in Sheared Emulsions: Role of Finite-Size Effects," in Nonlinear Dynamics in Polymeric Systems, edited by J. Pojman and Q. Tran-Cong (Washington, D.C.: American Chemical Society, 2003).

F.R. Phelan and N.S. Martys, "Simulation of Droplet Breakup Using a Lattice Boltzmann Method," in Proceedings of the Annual Technical Meeting of the Society of Plastics Engineers, 2340-2344, May 2003, Nashville, TN.

Y. Son, N.S. Martys, J.G. Hagedorn, and K.B. Migler, "Suppression of Capillary Instability of a Polymeric Thread via Parallel Plate Confinement," Macromolecules 36, 5825-5833 (2003).

Y. Son and K.B. Migler, "Interfacial Tension Measurement Between Immiscible Polymers: Improved Deformed Drop Retraction Method," Polymer 43, 3001-3006 (2002).

H. Wang, M.P. Nieh, E.K. Hobbie, C.J. Glinka, and J. Katsaras, "Kinetic Pathway of the Bilayer-Micelle to Perforated-Lamellae Transition," Physical Review E, Rapid Communication 67, 060902(R) (2003).

\section{Nanocomposite Processing}

A.J. Bur and M. McBrearty, "A Dielectric Slit Die for In-line Monitoring of Polymer Compounding," in Proceedings of the Annual Technical Meeting of the Society of Plastics Engineers, 3321-3325, May 2003, Nashville, TN.

A.J. Bur and S.C. Roth, "Fluorescence Temperature Measurements: Methodology for Applications to Processing," Polymer Engineering and Science, (in press). 
A.J. Bur, S.C. Roth, and M. McBrearty, "Monitoring Polymer/Clay Nanocomposite Compounding Using a Dielectric Slit Die," in Proceedings of the Annual Technical Meeting of the Society of Plastics Engineers, 3326-3330, May 2003, Nashville, TN.

C.H. Davis, L.J. Mathias, J.W. Gilman, D.A. Schiraldi, J.R. Shields, P. Trulove, T.E. Sutto, and H.C. Delong, "The Effects of Melt Processing Conditions on the Quality of Poly(ethylene terephthalate) Montmorillonite Clay Nanocomposites," Journal of Polymer Science, Part B, Polymer Physics 40, 2661-2666 (2002).

V. Ferreiro, G. Schmidt, C.C. Han, and A. Karim, "Dispersion and Nucleating Effects of Clay Fillers in Nanocomposite Polymer Films," in Polymer Nanocomposites: Synthesis, Characterization and Modeling, edited by R. Krishnamoorti and R. Vaia (Washington, D.C.: American Chemical Society, 2002) Chap. 14, pp. 177-191.

S.C. Glotzer and F.W. Starr, "Multiscale Modeling of Filled and Nanofilled Polymers," Proceedings of Foundations of Molecular Modeling and Simulation, (in press).

L. Granasy, T. Pusztai, J.A. Warren, J.F. Douglas, T. Borzsonyi, and V. Ferreiro, "Growth of 'Dizzy Dendrites' in a Random Field of Foreign Particles," Nature Materials 2, 92-96 (2003).

E.K. Hobbie, H. Wang, H. Kim, C.C. Han, E.A. Grulke, and J. Obrzut, "Optical Measurements of Structure and Orientation in Sheared Carbon-Nanotube Suspensions," Review of Scientific Instruments 74, 1244-1250 (2003).

E.K. Hobbie, H. Wang, H. Kim, S. Lin-Gibson, and E.A. Grulke, "Orientation of Carbon Nanotubes in a Sheared Polymer Melt," Physics of Fluids 15, 1196-1202 (2003).

Y. Jiang, A. Saxena, T. Lookman, and J.F. Douglas, "Influence of Filler Particles and Clusters on Phase Separation in Binary Polymer Blends," Journal of the Materials Research Society, (in press).

T. Kashiwagi, E. Grulke, J. Hilding, R. Harris, A. Awad, and J.F. Douglas, "Thermal Degradation and Flammability Properties of Poly(propylene)/Carbon Nanotube Composites," Macromolecular Rapid Communications 23, 761-765 (2002).

S. Lin-Gibson, G. Schmidt, J. Pathak, and C.C. Han, "Rheology of Poly(ethylene oxide)/Clay Solutions," ACS Polymeric Materials: Science and Engineering 87, 14-15 (2002).
S. Salaniwal, S.K. Kumar, and J.F. Douglas, "Amorphous Solidification in Polymer-Platelet Nanocomposites," Phyical Review Letters 89, art. no. 258301 (2002).

H. Wang and E.K. Hobbie, "Amphiphobic Carbon Nanotubes as Macroemulsion Surfactants," Langmuir 19, 3091-3093 (2003).

\section{Multivariant Measurement Methods}

\section{Combinatorial Methods Development}

E.J. Amis, X.D. Xiang, and J.C. Zhao, "Combinatorial Materials Science: What's New Since Edison?" MRS Bulletin 27, 295-300 (2002).

K.L. Beers, J.F. Douglas, E.J. Amis, and A. Karim, "Combinatorial Measurements of Crystallization Growth Rate and Morphology in Thin Films of Isotactic Polystyrene," Langmuir 19, 3935-3940 (2003).

M.Y.M. Chiang, R. Song, J. He, A. Karim, W.L. Wu, and E.J. Amis, "Combinatorial Edge Delamination Test for Thin Film Adhesion - Concept, Procedure and Results," in 3rd European Structural Integrity Society Conference on Fracture of Polymers, Composites and Adhesives, September 2003, Les Diablerets, Switzerland, (in press).

M.Y.M. Chiang, R. Song, A. Karim, and E.J. Amis, "Multivariant Measurement for Thin Film Adhesion as a Function of Temperature and Thin Film Thickness," in Nano Tech 2003, February 2003, San Francisco, CA.

M.Y.M. Chiang, W.L. Wu, J. He, and E.J. Amis, "Combinatorial Approach to the Edge Delamination Test for Thin Film Reliability," Thin Solid Films 437, 197-203 (2003).

A.J. Crosby, A. Karim, and E.J. Amis, "Combinatorial Investigations of Interfacial Failure," Journal of Polymer Science Part B - Polymer Physics 41, 883-891 (2003).

C.H. Davis, K.L. Beers, A.M. Forster, C.M. Stafford, A.P. Smith, C. Harrison, W. Zhang, A. Karim, and E.J. Amis, "Polymer Science at the NIST Combinatorial Methods Center," ACS Polymeric Materials: Science and Engineering 88, 386-387 (2003).

A.M. Forster, C. Stafford, A. Karim, and E.J. Amis, "Combinatorial Adhesion Measurement: Data Collection and Library Evaluation," ACS Polymeric Materials: Science and Engineering 88, 490-491 (2003). 
C. Harrison, J.A. Dagata, and D.H. Adamson, "Lithography with Self-Assembled Copolymer Microdomains," in Developments in Block Copolymer Science and Technology, (in press).

J.D. Hewes, D. Kaiser, A. Karim, and E.J. Amis, "Combinatorial Methods for Materials Research and Development - Contribution to Section on "Polymeric Materials," in Encyclopedia of Chemical Technology, (Kirk-Othmer Online, Wiley Interscience), (in press).

A. Karim, A. Sehgal, J.C. Meredith, A.J. Crosby, and E.J. Amis, "Image Analysis for High-Throughput Materials Science," in High Throughput Analysis A Tool for Combinatorial Materials Science, edited by R.A. Potyrailo and E.J. Amis (New York: Kluwer Academic/Plenum Publishers), Chap. 3, (in press).

J.C. Meredith, A.P. Smith, A.J. Crosby, E.J. Amis, and A. Karim, "Combinatorial Methods for Polymer Science," in Encyclopedia of Polymer Science and Technology, (New York: John Wiley \& Sons, Inc., 2002).

J.C. Meredith, A.P. Smith, A. Karim, and E.J. Amis, "Combinatorial Polymer Science: Synthesis and Characterization," in ACS Symposium Series, edited by Ripudaman Malhotra (Washington, D.C.: American Chemical Society, 2002) Chap. 2.

S.V. Roberson, A. Sehgal, A. Fahey, and A. Karim, "Time-Of-Flight Secondary Ion Mass Spectrometry (Tof-SIMS) For High-Throughput Characterization Of Biosurfaces," Applied Surface Science 203-204, 855-858 (2003).

C.M. Stafford, A.M. Forster, C.K. Harrison, C.H. Davis, E.J. Amis, and A. Karim, "High-Throughput Measurements of Polymer Adhesion and Mechanical Properties," in ACS Polymeric Materials: Science and Engineering, September 2003, New York, (in press).

C.M. Stafford, C. Harrison, A. Karim, and E.J. Amis, "Measuring Modulus of Gradient Polymer Films by Strain-Induced Bucking Instabilities," ACS Polymer Preprints 43, 1335 (2002).

\section{Surface and Polymer Interactions}

K.M. Ashley, J.C. Meredith, E. Amis, D. Raghavan, and A. Karim, "Combinatorial Investigation of Dewetting: Polystyrene Thin Films on Gradient Hydrophilic Surfaces," Polymer 44, 769-772 (2003).

K.M. Ashley, A. Sehgal, E.J. Amis, D. Raghavan, and A. Karim, "Combinatorial Mapping of Polymer Film Wettability on Gradient Energy Surfaces," Materials Research Society Symposium Proceedings on Organic/Biological Materials and Devices: Polymeric
Interfaces and Thin Films 700, S4.7.1-6 (2002).

J.T. Elliot, D.L. Burden, J.T. Woodward, A. Sehgal, and J.F. Douglas, "Phospholipid Monolayers Supported on Spun Cast Polystyrene Films," Langmuir 19, 2275-2283 (2003).

M.J. Fasolka, L.S. Goldner, J. Hwang, A.M. Urbas, P. DeRege, T. Swager, and E.L. Thomas, "Measuring Local Optical Properties: Near-Field Polarimetry of Photonic Block Copolymer Morphology," Physical Review Letters 90, 016107-1:4 (2003).

A.M. Forster and S.M. Kilbey, "The Influence of Shearing Parameters on the Friction Force Measured Between Polymer Brushes," ACS Polymer Preprints 44, 502-503 (2003).

S.N. Goldie, M.J. Fasolka, L.S. Goldner, J. Hwang, and K.L. Beers, "Near-Field Polarimetric Characterization of Semi-Crystalline Polymer Systems," ACS Polymeric Materials: Science and Engineering 88, 145-146 (2003).

L.S. Goldner, M.J. Fasolka, S. Nougier, H.P. Nguyen, G.W. Bryant, J. Hwang, K.D. Weston, K.L. Beers, A. Urbas, and E.A. Thomas, "Fourier Analysis Near-Field Polarimetry for Measurement of Local Optical Properties of Thin Films," Applied Optics 42, 3864-3881 (2003).

X. Gu, D. Raghavan, J.F. Douglas, and A. Karim, "Hole Growth Instability in the Dewetting of Evaporating Polymer Solution Films," Journal of Polymer Science, Part B - Polymer Physics 40, 2825-2832 (2002).

X. Gu, M.R. VanLandingham, M. Fasolka, J.W. Martin, J.Y. Jean, and T. Nguyen, "Enhancing Sensitivity of Atomic Force Microscopy for Characterizing Surface Chemical Heterogeneity," in Proceedings of the 26th Annual Meeting of the Adhesion Society, Inc., "Adhesion Fundamentals: From Molecules to Mechanisms and Modeling," 185-187, February 2003, Myrtle Beach, SC.

J.C. Meredith, J.L. Sormana, B.G. Keselowsky, A.J. Garcia, A. Tona, A. Karim, and E.J. Amis, "Combinatorial Characterization of Cell Interactions with Polymer Surfaces," Journal of Biomedical Materials Research, (in press).

A. Sehgal, V. Ferreiro, J.F. Douglas, E.J. Amis, and A. Karim, "Pattern-Directed Dewetting of Ultrathin Polymer Films," Langmuir 18, 7041-7048 (2002).

A.P. Smith, A. Sehgal, J.F. Douglas, A. Karim, and E.J. Amis, "Combinatorial Mapping of Surface Energy Effects on Diblock Copolymer Thin Film Ordering," Macromolecular Rapid Communications 24, 131-135 (2003). 


\section{Polymers Division}

\section{Chief}

Eric J. Amis

Phone: 301-975-6762

E-mail: eric.amis@nist.gov

\section{Deputy Chief}

Chad R. Snyder

Phone: 301-975-4526

E-mail: chad.snyder@nist.gov

\section{Senior Scientist}

\section{Wen-li Wu}

Phone: 301-975-6839

E-mail: wen-li.wu@nist.gov

\section{Group Leaders}

Characterization and Measurement Method Development/Internal Capabilities

Chad R. Snyder

Standards and Data

Bruno M. Fanconi

Phone: 301-975-6769

E-mail: bruno.fanconi@nist.gov

Electronics Materials

Eric K. Lin

Phone: 301-975-6743

E-mail: eric.lin@nist.gov

Biomaterials

Newell R. Washburn

Phone: 301-975-4348

E-mail: newell.washburn@nist.gov

Processing Characterization Kalman Migler

Phone: 301-975-4876

E-mail: kalman.migler@nist.gov

Multivariant Measurement Methods Alamgir Karim

Phone: 301-975-6588

E-mail: alamgir.karim@nist.gov 


\section{Research Staff}

Amis, Eric J.

eric.amis@nist.gov

Neutron, x-ray and light scattering

Polyelectrolytes

Viscoelastic behavior of polymers

Dendrimers and dendritic polymers

Functional biomaterials

\section{Anopchenko, Oleksiy S. ${ }^{+}$}

oleksiy.anopchenko@nist.gov

Dielectric relaxation spectroscopy

X-ray scattering

Microwave spectroscopy techniques

Antonucci, Joseph M.

joseph.antonucci@nist.gov

Synthetic and polymer chemistry

Dental composites, cements and adhesion

Initiator systems

Interfacial coupling agents

Remineralizing polymer systems

Arnould, Mark

mark.arnould@nist.gov

Mass spectrometry

Free-radical polymerization

Ashley, Karen ${ }^{+}$

karenmashley@hotmail.com

Polymer thin films

Dewetting

Combinatorial measurements

Balizer, Edward $^{+}$

Neutron, $x$-ray scattering

Polymer electrostriction

Bailey, LeeAnn O. $^{+}$

leeann.bailey@nist.gov

Cell biology

Apoptosis

Inflammatory responses

Flow cytometry

Polymerase chain reaction

Bauer, Barry J.

barry.bauer@nist.gov

Polymer synthesis thermal characterization

Neutron, $\mathrm{x}$-ray and light scattering

Dendrimers, metallic ions nanocluster

Porous low-k thin film characterization
Becker, Matt

matt.becker@nist.gov

Polymer synthesis

Block copolymers

Peptide synthesis

Phage display

Combinatorial methods

Beers, Kathryn

kathryn.beers@nist.gov

Crystallization in thin films

Optical light scattering

Controlled/living polymerizations

Polymer formulations

Polymer synthesis

Bencherif, Sidi ${ }^{+}$

sidi.bencherif@nist.gov

Mass spectrometry

Chemical modification

\section{Blair, William R.}

william.blair@nist.gov

Polymer analysis by size exclusion chromatography

Mass spectrometry of polymers

High temperature viscometry

Rayleigh light scattering

Bowen, Rafael L.*

rafael.bowen@nist.gov

Adhesion

Dental composites

Novel monomer synthesis

Bur, Anthony J.

anthony.bur@nist.gov

Dielectric properties of polymers

Fluorescence and optical monitoring of polymer processing

Piezoelectric, pyroelectric polymers

Viscoelastic properties of polymers

Byrd, H. Michelle

michelle.byrd@nist.gov

Mass spectrometry of polymers

Nuclear Magnetic Resonance

Cabral, $\mathrm{Joao}^{+}$

joao.cabral@nist.gov

Polymeric rapid prototyping

Polymer phase separation

Millifluidic measurements 


\section{Carey, Clifton M.*}

clifton.carey@nist.gov

Dental plaque

Microanalytical analysis techniques

Fluoride efficacy for dental health

De and remineralization

Phosphate chemistry

Ion-selective electrodes

\section{Cherng, Maria*}

maria.cherng@nist.gov

Calcium phosphate biomaterials

\section{Chiang, Chwan K.}

c.chiang@nist.gov

Electroluminescent polymers

Residual stress

Impedance spectroscopy

\section{Chiang, Martin Y.}

martin.chiang@nist.gov

Computational mechanics (finite element analysis)

Strength of materials, fracture mechanics

Engineering mechanics of polymer-based materials

Bi-material interface

\section{Chiche, Arnaud ${ }^{+}$}

arnaud.chiche@nist.gov

Adhesion on rough surfaces

Cavitation in peel test

Polymer adhesion

\section{Chow, Laurence C.*}

laurence.chow@nist.gov

Calcium phosphate compounds and biomaterials

Dental and biomedical cements

Solution chemistry

Topical dental fluorides

\section{Cicerone, Marcus T.}

marcus.cicerone@nist.gov

Protein stabilization

Glass transition theory

Optical coherence microscopy

Tissue engineering scaffolds

Confocal microscopy

\section{Cooper, James A.}

james.cooper@nist.gov

Tissue engineering

Polymer scaffolds

Cell biology

Optical microscopy

\section{Cygan, Zuzanna ${ }^{+}$}

\section{zcygan@umich.edu}

Polymer formulations

Florescent probe studies

Millifluidics of polymer solutions

Combinatorial and high-throughput methods
Davis, Cher

cher.davis@nist.gov

NIST Combinatorial Methods Center

Polymer nanocomposites

Polymers synthesis

Combinatorial methods and measurements

DeReggi, Aime S

aime.dereggi@nist.gov

Polarization-depth profiles in polymers

Space charge in dielectrics

Ferroelectric polymers

Polymeric piezo- \& pyroelectric devices

Biodegradation of polymers

Dickens, Sabine*

sabine.dickens@nist.gov

Dental composites

Dental adhesives

Transmission electron microscopy

Di Marzio, Edmund A. ${ }^{+}$

edmund.dimarzio@nist.gov

Statistical mechanics of polymers

Phase transitions

Glasses

Polymers at interfaces

Douglas, Jack F.

jack.douglas@nist.gov

Theory on polymer solutions, blends, and filled polymers

Transport properties of polymer solutions and polymers at interfaces

Scaling and renormalization group calculation

Conductivity/viscosity of nanoparticle filled systems

Crystallization of polymers

Duan, $\mathrm{Hu}^{+}$

hu.duan@nist.gov

Electron microscopy

Self-assembly and liquid crystals

Dunkers, Joy P.

joy.dunkers@nist.gov

Optical coherence microscopy

Image analysis

Fiber optic spectroscopy

Infrared microspectroscopy of polymers

Confocal fluorescence microscopy

Eichmiller, Frederick C.*

frederick.eichmiller@nist.gov

Clinical dentistry

Composites

Dentin adhesives 
Eidelman, Naomi N.* naomi.eidelman@nist.gov

Infrared mapping for surface chemistry

Characterization of calcified deposits by FTIR

Infrared microscopy

Fanconi, Bruno M.

bruno.fanconi@nist.gov

Infrared \& Raman spectroscopy of polymers

Structure of polymers

Polymer fracture

Process monitoring of polymer composites

Fasolka, Michael J.

mfasolka@nist.gov

Combinatorial methods and measurements

Nanopatterned substrates

Nanohybrid structures

Surface energy patterning and control

Surfaces and interfaces

Informatics standards

Scanning probe microscopy

Flaim, Glenn M.*

glenn.flaim@nist.gov

Fabricating dental composites

Floyd, Cynthia J. E.*

cynthia.floyd@nist.gov

Dental composites

Nuclear magnetic resonance

Flynn, Kathleen M.

kathleen.flynn@nist.gov

Melt flow rate measurements

Size exclusion chromatography

Mass spectrometry of polymers

Forster, Aaron M.

aaron.forster@nist.gov

Combinatorial methods and measurements

Polymer adhesion

Polymer interfaces

Biopolymer interactions

Fowler, Bruce O. $^{+}$

bruce.fowler@nist.gov

Infrared and Raman spectroscopy

Structure of calcium phosphates, bones, and teeth

Composites

Frukhtbeyn, Stanislav*

Calcium phosphate compounds and biomaterials

Topical dental fluorides

Galvin, Agnes Ly.*

agnes.galvin@nist.gov

Clinical dental assistant

Adhesion measurements
George, Laurie A.*

laurie.george@nist.gov

Network Administrator

Giuseppetti, Anthony A.*

anthony.giuseppetti@nist.gov

Casting of dental alloys

Scanning electron microcopy

Dental materials testing

Guttman, Charles M.

charles.guttman@nist.gov

Solution properties of polymers

Size exclusion chromatography

Mass spectrometry of polymers

Han, Charles C. . $^{+}$

charles.han@nist.gov

Phase behavior of polymer blends

Phase separation kinetics of polymer blends

Polymer characterization and diffusion

Shear mixing/demixing and morphology control of polymer blends

Static, time resolved, and quasi-elastic scattering

Harrison, Christopher

christopher.harrison@nist.gov

Combinatorial methods

Mechanical properties

Polymer thin film properties

Modulus measurements

Hedden, Ronald C.

ronald.hedden@nist.gov

Siloxane polymers: synthesis and properties

Nanoporous thin films

Gel permeation chromatography

Dendrimers

Hobbie, Erik K.

erik.hobbie@nist.gov

Light scattering and optical microscopy

Dynamics of complex fluids

Shear-induced structures in polymer blends and solutions

Holmes, Gale A.

gale.homes@nist.gov

Composite interface science

Chemical-structure-mechanical property

relationships for:

Polymer chemistry

Mass spectroscopy

Nanocomposites 
Hudson, Steven D.

steven.hudson@nist.gov

Electron microscopy

Polymeric surfactant and interfacial dynamics

Self-assembly and liquid crystals

Nanocomposites

Biomaterials

Hu, Tengjiao ${ }^{+}$

tengjiao.hu@nist.gov

Neutron, $x$-ray, and light scattering

Conformation of polymer chains in solution and at interfaces

Imperfections in crystals

Hussain, Latiff*

latiff.hussain@nist.gov

Monomer polymer synthesis, characterization, testing

Dental composites, adhesives

Jablonski, Erin L.

erin.jablonski@nist.gov

Near-edge x-ray absorption fine structure spectroscopy

Polymer diffusion, surfaces, thin films

Polymer phase behavior

Jones, Ronald L.

ronald.jones@nist.gov

Neutron and x-ray scattering

Neutron reflectivity

Polymer surfaces and thin films

Polymer phase transitions and computer simulation

Julthongpiput, Duangrut ${ }^{+}$

duangrut@hotmail.com

Combinatorial methods and measurements

Polymer adhesion and mechanical properties

Scanning probe microscopy

Kano, Kenj ${ }^{+}$

kkano@nist.gov

Dielectric relaxation of polymers

Nonlinear dielectric and conductive spectroscopy

\section{Kapeeleshwar, Krishna ${ }^{+}$}

kapeeleshwar.Krishana@us.rhodia.com

Polymer mixing strategies

Polymer coatings

Polymer formulations
Karim, Alamgir

alamgir.karim@nist.gov

Combinatorial and high-throughput methods

Combinatorial measurements

Patterning of thin-polymer blend films on inhomogenous surfaces

Neutron and x-ray reflection, scattering, AFM and optical microscopy

Thin-film phase behavior of polymer blends

Nanofilled polymer films

Kee, Tak $^{+}$

tak.kee@nist.gov

Ultrafast spectroscopy

CARS microscopy

Tissue engineering scaffolds

Confocal microscopy

Kennedy, Scott B.

scott.kennedy@nist.gov

Tissue engineering

Biomaterials

Block copolymers

Combinatorial surface patterns

Kharchenko, Semen ${ }^{+}$

semen.kharchenko@nist.gov

Stress optical properties

Birefringence

Viscoelastic properties

Khatri, Chetan A. ${ }^{+}$

chetan.khatri@nist.gov

Monomer/polymer synthesis and characterization

Khoury, Freddy A.

freddy.khoury@nist.gov

Crystallization, structure and morphology of polymers

Analytical electron microscopy of polymers

Wide-angle and small-angle $\mathrm{x}$-ray diffraction

Structure and mechanical property relationships

Kim, Jae Hyun ${ }^{+}$

jaehyun@nist.gov

Fiber/matrix interface

Mechanical properties

Polymer composites

Landis, Forrest A.

forrest.landis@nist.gov

Crystallization and melting of miscible polymer blends

Ionomers

Optical coherence microscopy

Tissue engineered scaffolds

Static small angle laser light scattering 
Lee, Hae-Jeong ${ }^{+}$

hae-jeong.lee@nist.gov

$\mathrm{X}$-ray reflectivity

Small-angle neutron scattering

Structural characterization of low dielectric constant thin films

Porosimetry of porous thin films/light-emitting diode (LED)

Lee, Yu-Hsin (Mandy) ${ }^{+}$

yu-hsin.lee@nist.gov

Dielectric relaxation spectroscopy

Nanocomposites

Atomic force microscopy

Rubber-toughened phenolic resins

Dynamic mechanical analysis of polymer blends

Lenhart, Joseph L. ${ }^{+}$

jllenha@sandia.gov

Sensors

Interphase structure

Polymer films and interfaces

Near-edge $\mathrm{x}$-ray absorption fine structure

$\mathrm{X}$-ray and neutron reflectivity

Liao, Nam S.*

nam.liao@nist.gov

Synthesis and testing of dental adhesives

Internal stress of composition due to

polymerization

Web page development

Lin, Eric K.

eric.lin@nist.gov

Polymer thin films and interfaces

Polymeric photoresists for lithography

Small angle neutron scattering

Statistical mechanics

$\mathrm{X}$-ray and neutron reflectivity

\section{Lin-Gibson, Sheng}

sheng.lin-gibson@nist.gov

Rheology of gels \& nanocomposites

Mass spectrometry of synthetic polymers

Polymer synthesis and modification

Structure and dynamics of nanocomposite polymeric materials

Liu, D.-W.

da-wei.liu@nist.gov

Polymer synthesis

Thermal gravimetric analysis

Differential scanning calorimetry

Gel permeation chromatography

Infrared spectroscopy

Nuclear magnetic resonance
Mager, Carie ${ }^{+}$

carie.mager@nist.gov

Toughening bioceramics

Fracture mechanics

Markovic, Milenko* milenko.markovic@nist.gov

Calcium phosphate chemistry

Biomineralization (normal and pathological)

Crystal growth and dissolution kinetics

Heterogeneous equilibria

Maurey, John R. ${ }^{+}$

john.maurey@nist.gov

Ultracentrifugation

Rayleigh light scattering

Osmometry

Densimetry

Refractometry

Intrinsic viscosity

McDonough, Walter G.

walter.medonough@nist.gov

Processing and cure monitoring polymer composites

Failure and fracture of polymers

Polymer composite interfaces

Dental materials

McGuiggan, Patricia

patricia.mcguiggan@nist.gov

Atomic force microscopy

Viscoelastic properties

Mei, Ying ${ }^{+}$

ying.mei@nist.gov

Polymer synthesis

Peptide synthesis

Biodegradable polymers

Biomimetic polymers

Migler, Kalman

kalman.migler@nist.gov

Effects of shear and pressure on phase behavior

Fluorescence and optical monitoring of polymer processing

Liquid crystals

Shear-induced two phase structures

Polymer slippage

Noda, Natsuko ${ }^{+}$

natsuko.noda@nist.gov

Impedence spectroscopy

Dielectric characterization of composites

Photolithography

Nanocomposites 
Norman, Alexander ${ }^{+}$

alexander.norman@nist.gov

Polymer formulations

Water soluble polymers

Microemulsions

Neutron and x-ray scattering from polymers

\section{Obrzut, Jan}

jan.obrzut@nist.gov

Dielectric relaxation spectroscopy

Electronic properties of polymers and composites

Electronic packaging

Microwave and optical waveguides

Photoelectron spectroscopy (x-ray and UV)

Reliability, stress testing

\section{Parry, Edward E.*}

edward.parry@nist.gov

Dental appliance and crown and bridges fabrication

Machine shop applications

Pathak, Jai A. ${ }^{+}$

jai.pathak@nist.gov

Polymer rheology

Polymer dynamics

Glass transition

Polymer blends

Pfeiffer, Carmen ${ }^{+}$

carmen.pfeiffer@nist.gov

Dental resins

Monomer synthesis

Phelan, Jr., Frederick R.

frederick.phelan@nist.gov

Resin transfer molding: modeling and processing studies

Viscoelastic flow modeling

Flow in porous media

Lattice Boltzmann methods

Prabhu, Vivek M.

vmprabhu@nist.gov

Small-angle neutron scattering

Polyelectrolytes

Light scattering

Fluorescence correlation spectroscopy

Polymer thin films

$\mathrm{X}$-ray and neutron reflectivity

Quinn, Janet*

janet.quinn@nist.gov

Fractography

Composites
Raghavan, Dharmaraj ${ }^{+}$

draghavan@howard.edu

Curing of epoxies

High-throughput and combinatorial measurements Confocal microscopy

Roth, Steven C.

steven.roth@nist.gov

Piezoelectric polymer transducers - fabrication and applications

Vacuum deposition of metals

Calibration of polymer transducers

Microcomputer interfacing

Fluorescence measurements

Schumacher, Gary E.*

gary.schumacher@nist.gov

Clinical dentistry

Composites

Dentin adhesives

Sehgal, Amit ${ }^{+}$

amit@nist.gov

Biomaterials, cell-surface biophysics

Polymer thin films

Polyelectrolytes and conducting polymers

Structure and dynamics of polymer solutions

Light scattering and fluorescence photobleaching recovery

Shimizu, Katsumi ${ }^{+}$

Polymer blends

Polymer crystallization and melting

Phase contrast optical microscopy

Silverstein, Michael S. ${ }^{+}$

michael.silverstein@nist.gov

Porous low-k dielectrics

Small angle neutron scattering

X-ray reflectivity

Plasma polymerization

Polymer films for electronic applications

Simon, Carl G., Jr.

carl.simon@nist.gov

Biocompatibility

Cytotoxicity

Signaling in human platelets

Bone marrow cell lineage/trafficking

\section{Skrtic, Drago*}

drago.skrtic@nist.gov

Amorphous calcium phosphates as dental materials 
Snyder, Chad R.

chad.snyder@nist.gov

Polymer crystallization

WAXD and SAXS of polymeric materials

Thermal expansion measurements

Thermal analysis of polymers

Dielectric measurements and behavior

Nanocomposite properties

Soles, Christopher L.

csoles@nist.gov

Polymer dynamics

Inelastic neutron scattering

Low-k dielectric thin films

$\mathrm{X}$-ray and neutron reflectivity techniques

Polymer thin films and lithography

Ion beam scattering techniques

Song, Rui ${ }^{+}$

rsong@nist.gov

Combinatorial methods and measurements

Polymer debonding

Polymer adhesion and failure

Polymeric peel tests

Stafford, Christopher

chris.stafford@nist.gov

Polymer adhesion

Surfaces and interfaces

Polymer thin films

Starr, Francis

francis.starr@nist.gov

Dynamics of filled polymers

Slow dynamics in soft condensed matter

Molecular dynamics simulations and parallel

Computational methods

Start, Paul R. ${ }^{+}$

paul.start@nist.gov

nanocomposites

TEM

Sol-gel processes

Surfactants and interfacial tension

Takagi, Shozo*

shozo.takagi@nist.gov

Crystallography

$\mathrm{X}$-ray diffraction

Calcium phosphate biomaterials

Topical fluoridation

De- and remineralization
Tesk, John A.

john.tesk@nist.gov

Biomaterials: industrial relations

Characterization: biomaterials; physical and mechanical properties

Reference biomaterials

Reference data for biomaterials

Biomaterials: orthopaedics, cardiovascular, dental, opthalmic, \& tissue engineered medical devices

Standards for medical devices

Tung, Ming S.*

ming.tung@nist.gov

Chemistry of calcium phosphate compounds

Remineralization studies

Standard reference materials

VanderHart, David L.

david.vanderhart@nist.gov

Measurement of orientation in polymer fibers and films

Solid-state NMR of polymers

Measurement of polymer morphology at the $2-50 \mathrm{~nm}$ scale

Pulsed field gradient NMR

Van Workum, Kevin

kevin.vanworkum@nist.gov

Monte Carlo and molecular dynamics simulations

Mechanical properties of glassy and crystalline structures

Dispersion in nanocomposites

Vogel, Gerald L.*

gerald.vogel@nist.gov

Dental plaque chemistry, chemistry of calcium phosphates

Microanalytical techniques

Vogt, Bryan D.

bryan.vogt@nist.gov

Polymer thin film properties

$\mathrm{X}$-ray and neutron reflectivity

Interfacial effects

Ordered mesoporous materials

Wallace, William E.

william.wallace@nist.gov

Mass spectrometry

Geometric data analysis methods

Walls, Howard J.

howard.walls@nist.gov

Rheology of polymers

Combinatorial measurements and methods

Viscoelastic polymers 


\section{Wang, Chia-Ying ${ }^{+}$}

cywang@nist.gov

Small-angle x-ray scattering

Polymer diffusion

Microphase transitions of block copolymers

Fluorescence spectroscopy of polymers

\section{Wang, Francis W.}

francis.wang@nist.gov

Photophysics and photochemistry of polymers

Fluorescence spectroscopy

Cure monitoring of polymerization

Tissue engineering

Wang, Howard ${ }^{+}$

hao.wang@nist.gov

Multiple phase transitions in polymer

Wetting, segregation and transport in polymer

Structure and dynamics in polymer gels

Wang, Michael X. ${ }^{+}$

mwang@nist.gov

Small angle $\mathrm{x}$-ray \& neutron scattering

$\mathrm{X}$-ray \& neutron reflectivity

Light scattering

Polymer thin films

Combinatorial methods

\section{Wang, Xianfeng ${ }^{+}$}

xianfeng.wang@nist.gov

Monte Carlo simulations

Finite element modeling

Polymer composites

Mechanical properties

\section{Washburn, Newell}

newell.washburn@nist.gov

Tissue engineering

Polymer blends

Biomaterial interfaces

Viscoelastic properties of polymers

Weir, Michael

michael.weir@nist.gov

Biomaterials

Tissue engineering

Biodegradable hydrogels

Combinatorial analysis

UV and fluorescence spectroscopy of polymers
Wetzel, Stephanie J.

stephanie.wetzel@nist.gov

Mass spectrometry of polymers

Chemometrics

Size exclusion chromatography

Wu, Wen-li

wen-li.wu@nist.gov

Neutron and x-ray scattering and reflectivity

Electron microscopy

Mechanical behavior of polymers and composites

Polymer surfaces and interfaces

$\mathrm{Wu}, \mathrm{Tao}^{+}$

tao.wu@nist.gov

Polymer formulations

Polymer synthesis

Interfacial tension measurements

Combinatorial and high-throughput methods

Yurekli, Koray $^{+}$

kyurekli@Jetson.uh.edu

Polymer nanocomposite

Phase separation

Thin films and interfaces

Neutron and light scattering

Xu, Huakun*

hockin.xu@nist.gov

Remineralizing composites

Wear and fatigue

Fiber and whisker reinforcement

Zhang, $\mathrm{Kai}^{+}$

kai.zhang@nist.gov

Polymer scaffolds

Tissue engineering

Dental resins

Combinatorial methods

Zhang, Wenhua ${ }^{+}$

wezhang@nist.gov

Combinatorial and high-throughput informatics

Database structure

Laboratory automation

Polymer thin films and blends

* Research Associate

+ Guest Scientist 



\section{Organizational Charts}

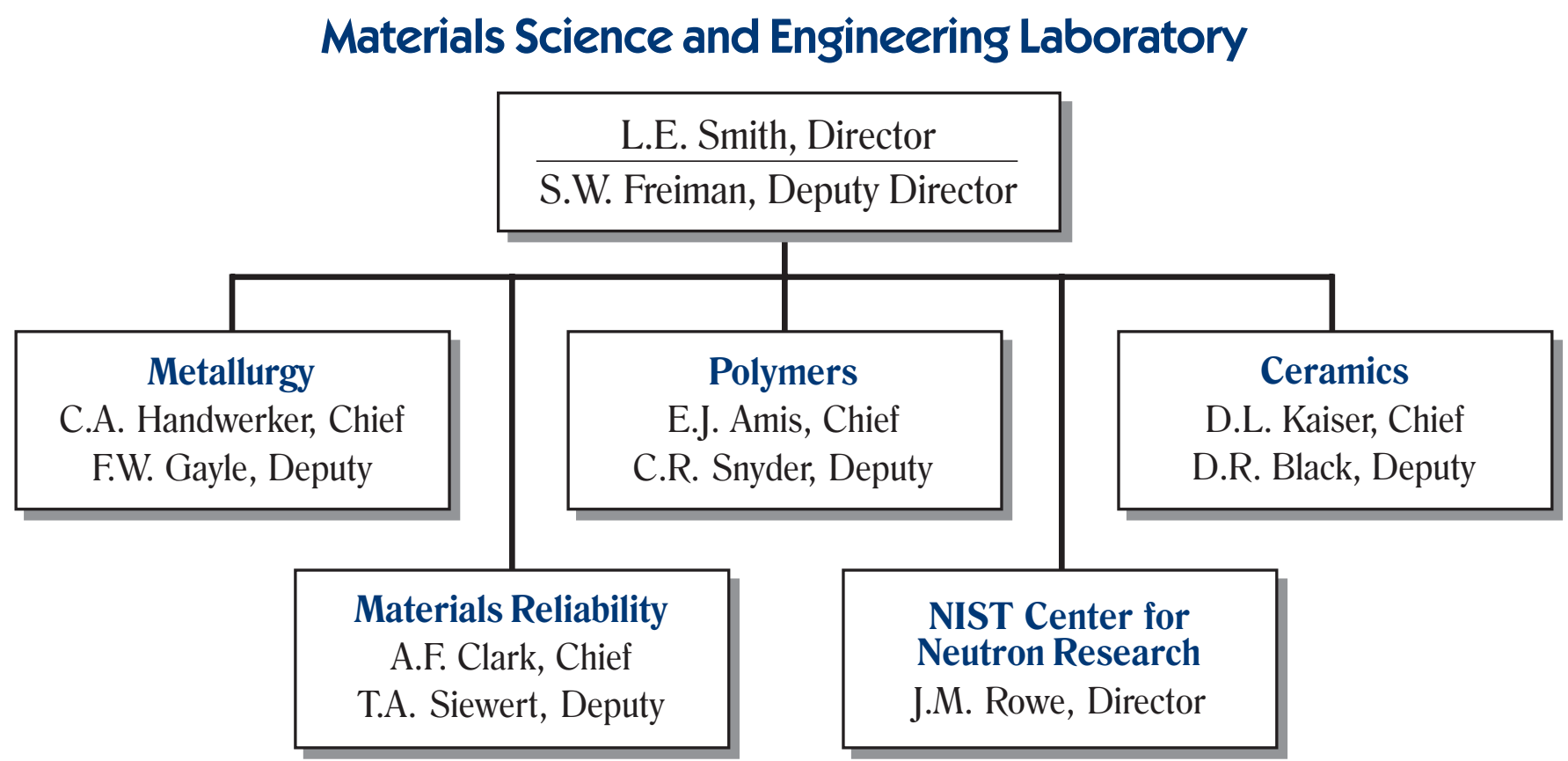

\section{National Institute of Standards and Technology}

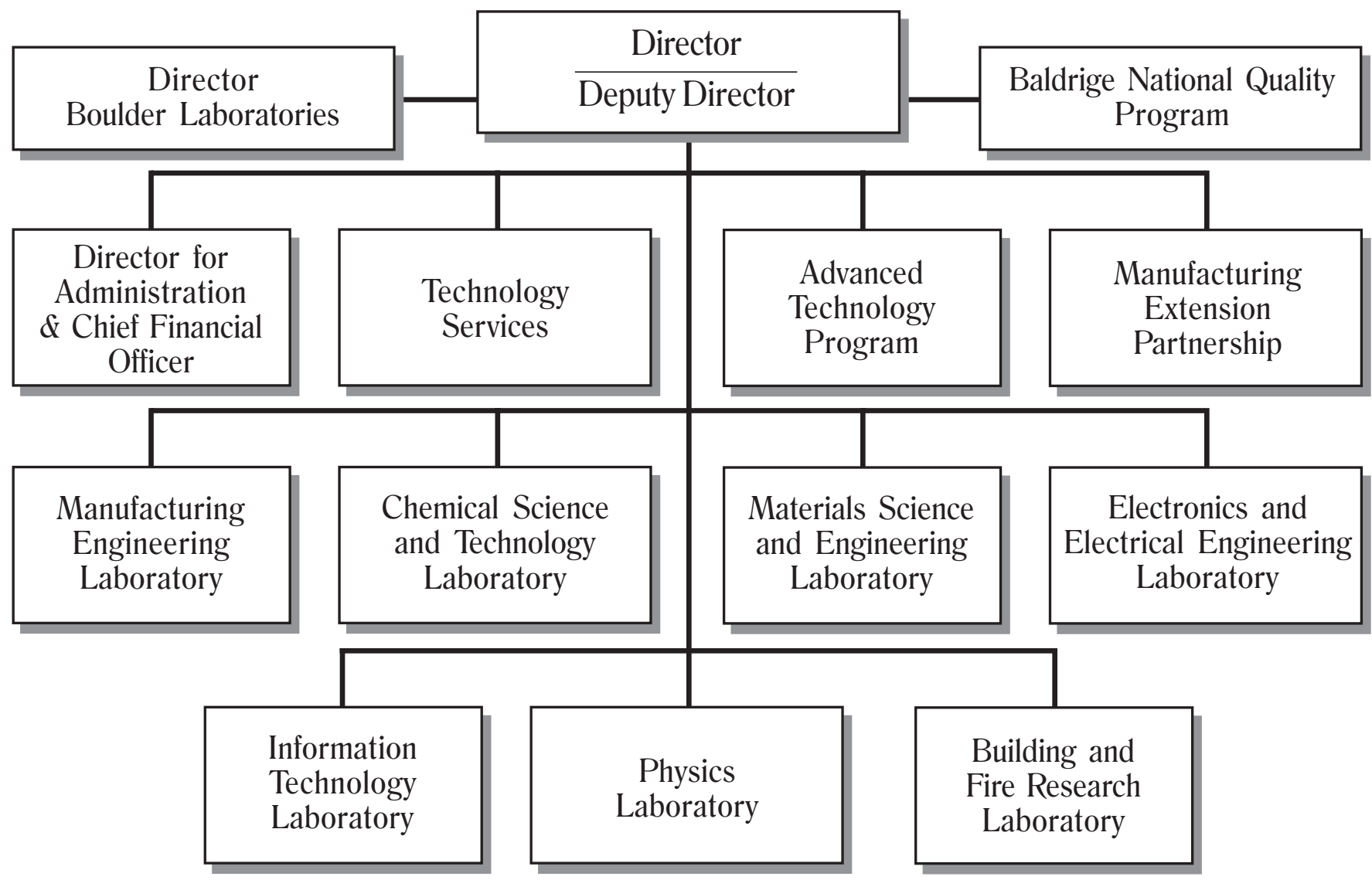


Polymers Division (854.00)

\begin{tabular}{c} 
Eric J. Amis, Chief \\
Chad R. Snyder, Deputy Chief \\
Wen-li Wu, Senior Scientist \\
Dawn Bradley, Division Secretary \\
Diane Fernandez, Administrative Officer \\
Lisa Fedders, Administrative Assistant \\
Linda Souders, Secretary \\
\hline
\end{tabular}

Characterization and Measurement

(854.01)

Chad R. Snyder, Leader

Bronny Webb, Secretary

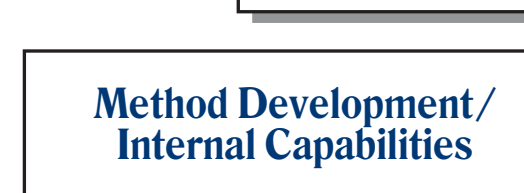

Chad R. Snyder, Leader
M. Chiang
F. Khoury
M. Cicerone
A. DeReggi
J. Douglas
J. Dunkers
R. Hedden
G. Holmes
F. Landis
D. Liu
W. McDonough
F. Starr
K. Van Workum
D. VanderHart

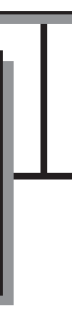

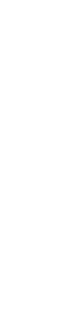

T. Kee

J. Kim

S. Liao

$\mathrm{X}$. Wang

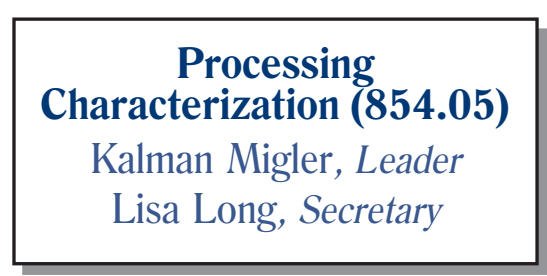

J. Antonucci L. Bailey

M. Becker

J. Cooper

S. Kennedy

K. O'Brien

C. Simon

F. Wang

M. Weir

K. Wilson
S. Bencherif

C. Khatri

Y. Mei

N. Richards

E. Schwartz

A. Sehgal

K. Zhang

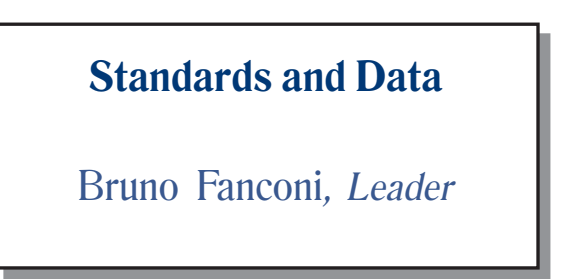
M. Arnould

W. Blair

M. Byrd

K. Flynn

C. Guttman

J. Tesk

W. Wallace

S. Wetzel

\section{Electronics Materials (854.02)}

Eric Lin, Leader

Bronny Webb, Secretary

\begin{tabular}{cc}
\hline B. Bauer & O. Anopchenko \\
C. Chiang & D. Ho \\
E. Jablonski & T. Hu \\
R. Jones & K. Kano \\
J. Obrzut & H. Lee \\
V. Prabhu & S. Sambasivan \\
C. Soles & \\
B. Vogt &
\end{tabular}

Multivariant Measurement Methods (854.06)

Alamgir Karim, Leader Lisa Long, Secretary
A. Bur
H. Duan
K. Beers K. Ashley

D. Fry

S. Kharchenko

E. Hobbie

G. Matsuba

S. Hudson

M. Meillon

S. Lin-Gibson

D. Morgan

P. McGuiggan

J. Pathak

F. Phelan

S. Roth
C. Davis J. Cabral

M. Fasolka A. Chiche

A. Forster Z. Cygan

C. Stafford K. Kapeeleshwar

H. Walls A. Norman

M. Wang

W. Zhang 

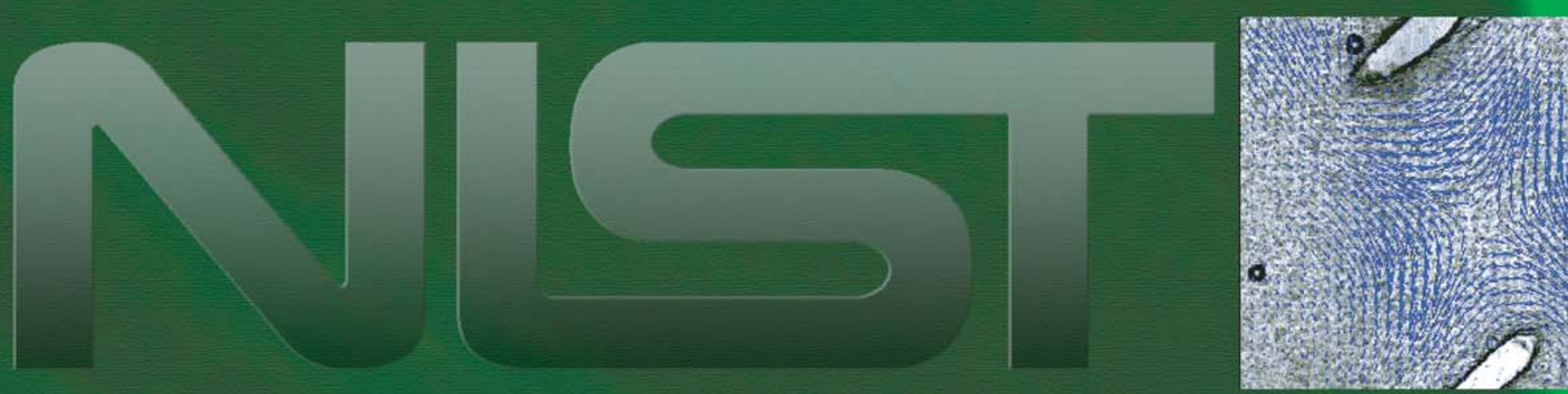